\title{
Flux and instanton effects in local F-theory models and hierarchical fermion masses
}

\author{
L. Aparicio, ${ }^{a, b}$ A. Font, ${ }^{c}$ L.E. Ibáñez ${ }^{a, b}$ and F. Marchesano ${ }^{b}$ \\ ${ }^{a}$ Departamento de Fúsica Teórica, Universidad Autónoma de Madrid, \\ Cantoblanco, 28049 Madrid, Spain \\ ${ }^{b}$ Instituto de Física Teórica UAM-CSIC, \\ C/ Nicolás Cabrera 13-15, Cantoblanco, 28049 Madrid, Spain \\ ${ }^{c}$ Departamento de Física, Centro de Física Teórica y Computacional, \\ Facultad de Ciencias, Universidad Central de Venezuela \\ Avenida los Ilustres, A.P. 20513, Caracas 1020-A, Venezuela \\ E-mail: luis.aparicio@uam.es, afont@fisica.ciens.ucv.ve, \\ luis.ibannez@uam.es, fernando.marchesano@csic.es
}

ABSTRACT: We study the deformation induced by fluxes and instanton effects on Yukawa couplings involving 7-brane intersections in local F-theory constructions. In the absence of non-perturbative effects, holomorphic Yukawa couplings do not depend on open string fluxes. On the other hand instanton effects (or gaugino condensation on distant 7-branes) do induce corrections to the Yukawas. The leading order effect may also be captured by the presence of closed string $(1,2)$ IASD fluxes, which give rise to a non-commutative structure. We check that even in the presence of these non-perturbative effects the holomorphic Yukawas remain independent of magnetic fluxes. Although fermion mass hierarchies may be obtained from these non-perturbative effects, they would give identical Yukawa couplings for D-quark and Lepton masses in SU(5) F-theory GUT's, in contradiction with experiment. We point out that this problem may be solved by appropriately normalizing the wavefunctions. We show in a simple toy model how the presence of hypercharge flux may then be responsible for the difference between D-quarks and Lepton masses in local SU(5) GUT's.

KEYwords: F-Theory, Quark Masses and SM Parameters, Nonperturbative Effects, GUT

ARXIV EPRINT: 1104.2609 


\section{Contents}

1 Introduction $\quad 2$

2 Wavefunctions and Yukawa couplings in local F-theory models 5

2.1 Local F-theory models from intersecting 7-branes 5

$\begin{array}{ll}2.2 & \text { Zero and massive modes at the intersection } \\ \end{array}$

2.3 A U(3) toy model 8

$\begin{array}{llr}2.4 & \text { Yukawa couplings } & 12\end{array}$

3 Non-perturbative effects on intersecting 7-branes $\quad 14$

$\begin{array}{lll}3.14 \mathrm{~d} \text { approach } & 15\end{array}$

$\begin{array}{lll}3.2 & 8 \mathrm{~d} \text { approach } & 20\end{array}$

$\begin{array}{lll}3.3 & \text { Non-commutative approach } & 25\end{array}$

4 Corrected wavefunctions $\quad 26$

$\begin{array}{lll}4.1 & \text { Wavefunctions and perturbation theory } & 27\end{array}$

$\begin{array}{lll}4.2 & \text { Relating commutative and non-commutative wavefunctions } & 29\end{array}$

5 Wavefunctions and Yukawas in the non-commutative formalism 32

5.1 Non-commutative zero modes in the U(3) toy model 32

5.1.1 Zero modes with $\theta$ coordinate dependent 36

$\begin{array}{lll}5.2 & \text { Non-commutative Yukawa couplings } & 37\end{array}$

$6 \quad$ Flux dependence, D-terms and Yukawa couplings $\quad 39$

6.1 The $Y(D)=Y(L)$ problem $\quad 39$

6.2 Normalization and flux dependence in physical couplings 40

6.3 A model of quark-lepton hierarchies 41

$7 \quad$ Final comments and conclusions $\quad 43$

A Magnetized D9-branes and KK modes 46

A.1 Massive modes for the U(3) model 49

$\begin{array}{lll}\text { A.2 Fermionic conventions } & 54\end{array}$

B Computing wavefunctions in the commutative formalism $\quad 56$

B.1 $\beta$-deformed wavefunctions and massive modes $\quad 56$

$\begin{array}{ll}\text { B.2 The U(3) model } & 57\end{array}$

C Equivalence of commutative and non-commutative actions 60 


\section{Introduction}

If string theory underlies the physical world [1] it should be able to describe the observed structure of fermion masses and mixings, governed by the Yukawa couplings. In type IIB orientifold compactifications Yukawas at tree level may in principle be computed as overlap integrals of three different wavefunctions over the compact dimensions. In practice those wavefunctions are only known in some simple examples, like toroidal orientifolds. For instance, in $\mathbf{T}^{\mathbf{6}} / \mathbf{Z}_{\mathbf{2}} \times \mathbf{Z}_{\mathbf{2}}$ orientifolds with magnetized D7-branes one can construct semirealistic models $[2,3]$ in which the Yukawas can be explicitly computed by integration of three overlapping wavefunctions. In these examples the wavefunctions are given by certain Jacobi $\vartheta$-functions and the resulting mass matrices have rank one, corresponding to a single massive quark/lepton generation [4]. This is a good starting point, and one hopes that further effects like string instanton may yield masses for the lightest generations [5].

More generally we would like to be able to compute Yukawa couplings in more complicated curved geometries which may arguably be needed to obtain more realistic models. This may be less difficult than it sounds. In the context of intersecting D7-brane models bifundamental matter fields reside at pairs of D7-branes intersecting in Riemann curves, and Yukawa couplings appear locally at those points where three of these curves intersect. Thus one might hope to be able to compute the corresponding Yukawa coupling in terms of just local information around the triple intersection point.

This possibility is particularly attractive in the context of local F-theory GUT models [6-9], in which there is a 4-cycle $S$ on which the GUT 7-branes wrap and the matter fields reside at the so-called matter curves $\Sigma_{i} \subset S$ at which the GUT symmetry is enhanced (see $[10,11]$ for recent reviews). In the $\mathrm{SU}(5)$ case the symmetry is enhanced to $\mathrm{SU}(6)$ at curves with 5-plets and to $\mathrm{SO}(10)$ at curves with 10-plets. In addition, these curves intersect at points at which the symmetry is further enhanced, and which give rise to Yukawa couplings among fields in the matter curves. In particular the $\mathbf{1 0} \times \overline{\mathbf{5}} \times \overline{\mathbf{5}}_{\mathbf{H}}$ Yukawa appears at points with enhanced $\mathrm{SO}(12)$ symmetry and the $\mathbf{1 0} \times \mathbf{1 0} \times \mathbf{5}_{\mathbf{H}}$ appears at points of enhanced $E_{6}$ symmetry. Locally one may describe these couplings in terms of three intersecting curves where the internal wavefunctions of the zero modes are peaked, and so compute the corresponding Yukawa couplings in terms of the overlapping integral of the three wavefunctions over $S[7,8,12-18]$. Since the wavefunctions are localized along the matter curves, the Yukawa coupling is expected to depend only on the data around the neighborhood of the intersection point, and not much on the full structure of the compact space. This is an F-theory realization of the bottom-up idea for the embedding of the Standard Model in string theory [19].

It turns out that, assuming that there is only one intersecting point for each of the two types of SU(5) couplings, the resulting Yukawa matrices have rank equal to one [13]. ${ }^{1}$ Hence only one generation gets massive, similarly to the toroidal orientifolds mentioned above. It was first thought that the dependence of the Yukawa couplings on the worldvolume fluxes on the 7-branes (i.e., those required for obtaining chirality from these settings), could correct this result and so give masses to the rest of quarks and leptons. However

\footnotetext{
${ }^{1}$ For different approaches to fermion hierarchies in F-theory and type IIB models see [20-22].
} 

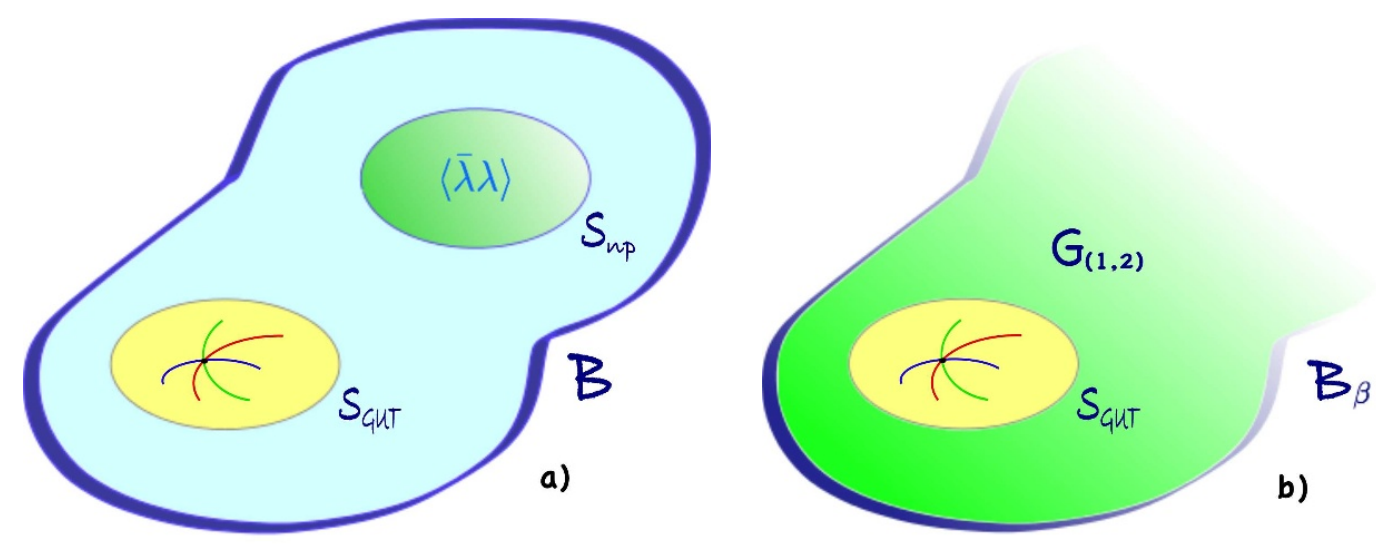

Figure 1. Sources of corrections to 7-brane Yukawas. Figure a) represents the setup considered in [23], where the Yukawas on a 7-brane stack wrapping the four-cycle $S_{G U T}$ are modified by the gaugino condensate on 7-branes on the distant four-cycle $S_{\text {np }}$. Following [25], one may identify this setup with the one in figure $b$ ), where the non-perturbative sector has been replaced by a $\beta$-deformation of the previous background. This new background contains IASD $(1,2)$ background fluxes that induce a non-commutative deformation on $S_{G U T}$, in the sense of [16]. This $\beta$-deformation is usually not-well defined around $S_{\mathrm{np}}$, and so typically the new three-fold $B_{\beta}$ can only be defined locally.

it was soon realized that open string fluxes by themselves are not enough, since they do not modify the rank of the Yukawa matrices [15-17]. In particular, one can see that the F-term zero mode equations become independent of the worldvolume fluxes in a certain holomorphic gauge [15] and that, as a consequence, the holomorphic Yukawa couplings remain flux independent.

Two possible sources of corrections to the holomorphic Yukawas were then put forward. It was first found that a non-commutative deformation of the 7-brane gauge theory can induce corrections to the Yukawas such that the rank of the mass matrix is modified [16]. Such deformation can be generated by placing D7-branes on type IIB backgrounds with closed string IASD fluxes of the (1,2)-type, often referred to as $\beta$-deformed backgrounds. ${ }^{2}$ The other possible source is the influence of non-perturbative (instanton or gaugino condensate) effects on distant 4-cycles in the compact manifold [23], see figure 1. Although these two proposals look quite different they lead to similar physics and it was pointed out that they should be equivalent, the reason being that instanton and gaugino condensate effects source IASD $(1,2)$ fluxes on the theory [24] (see also [25]). However, a detailed comparison of both kind of effects for the dynamics of local F-theory models is still lacking.

The purpose of this paper is threefold. On the one hand we revisit these two different sources of corrections for the Yukawa couplings in local F-theory GUT constructions and

\footnotetext{
${ }^{2}$ Such type IIB backgrounds are rather exotic, in the sense that they contain at least one harmonic 1-form and that D3-branes develop a non-trivial superpotential at tree level. As a result, their F-theory lift does not correspond to a Calabi-Yau four-fold compactification. In fact, to date no compact example of $\beta$-deformed type IIB background has been found.
} 
study their relationship. After a detailed study of the local equations of motion, we show that there is an explicit Seiberg-Witten map which relates the non-commutative and nonperturbative equations at leading order in the perturbation.

Secondly, we study these corrections in detail for the simplest rank two enhancing example allowing for Yukawa couplings, a U(3) model sufficient to capture the main ingredients of the group theoretically more involved situation in GUT's constructions. We obtain the local wavefunctions to first order in the perturbation and compute the corresponding holomorphic Yukawas for the case of constant magnetic fluxes and a perturbation linear in the local coordinates. The result shows an interesting hierarchical structure but is again independent of the worldvolume fluxes. This is a general property of the setting, and not an artifact of any particular model.

Finally, we emphasize a problematic phenomenological consequence of this persistent flux independence of the holomorphic couplings, what we dub the $Y(D)=Y(L)$ problem. Indeed, in a SU(5) GUT the D-quark and lepton Yukawas are identical, namely $Y^{i j}(D)=$ $Y^{i j}(L)$ with $i, j$ family labels. Although the related prediction for the heaviest generation is consistent with data, those for the lightest generations are definitely wrong. In F-theory models, the hope is that such mass difference for the lightest generations can be understood in terms of the hypercharge worldvolume flux, which is the only ingredient breaking the GUT symmetry down to the MSSM. Now, if the Yukawa couplings are flux independent even after non-perturbative corrections, that possibility disappears. All is however not lost. We point out that the wavefunctions used should be normalized and it is this fact which brings back again the flux dependence for the physical (not the holomorphic) Yukawas. Using this example as a toy model for $\mathrm{SU}(5)$ we find that indeed this structure of flux dependence may explain the hierarchy of masses and mixings of the three quark-lepton generations. Wavefunction normalization would thus be the only difference between Dquark and lepton masses at a fundamental level (in addition to the different low-energy running). Although in a different form, the idea that hypercharge flux could split the leptonic and down type Yukawas was considered in [13].

The detailed structure of this paper is as follows. In section 2 we describe the computation of wavefunctions and Yukawas for local F-theory models in the absence of any non-perturbative or non-commutative deformation, illustrating the general computation by means of an explicit $\mathrm{U}(3)$ local toy model. In section 3 we discuss how the previous scheme is modified in the presence of non-perturbative effects, and describe three different approaches that lead to the same set of corrected Yukawa couplings. In particular, we consider two different approaches based on the computation of zero mode wavefunctions for $8 \mathrm{~d}$ fields living on the GUT 7-brane worldvolume, one of them related to a commutative $8 \mathrm{~d}$ gauge theory and the other to a non-commutative one. The corresponding zero mode equations and wavefunctions look different, but are elegantly related by a Seiberg-Witten map, as described in section 4. Both approaches being equivalent, in section 5 we focus on the non-commutative formalism, in which the Yukawa independence on the worldvolume fluxes is manifest. There we compute explicitly the corrected zero modes and Yukawas for the U(3) model previously introduced, and show that the Yukawas have a clear hierarchical structure. Finally, in section 6 we discuss the phenomenological implications of this type 
of Yukawa structure, and more precisely how to solve the $Y^{i j}(D)=Y^{i j}(L)$ problem. We conclude in section 7 and mention some important remarks regarding the generalization of this scheme to concrete F-theory GUT constructions.

Several technical details and computations have been left for the appendices. In appendix A we provide a dual description of intersecting D7-brane models in terms of magnetized D9-branes. This dual description allows to easily compute not only the spectrum of wavefunctions for the chiral modes of the configuration, but also for their massive replicas, as we show for the $\mathrm{U}(3)$ model. In appendix B we compute the corrected zero mode wavefunctions for the $\mathrm{U}(3)$ model in the commutative formalism, using a slightly different strategy from section 5. Finally, appendix $\mathrm{C}$ shows the equivalence of the commutative and non-commutative formalisms at the level of the superpotential.

\section{Wavefunctions and Yukawa couplings in local F-theory models}

In this section we describe the standard computation of wavefunctions and Yukawas for local F-theory models in the absence of any non-perturbative or non-commutative deformation, illustrating the general computation by means of an explicit $\mathrm{U}(3)$ toy model. While the discussion below is fully carried out in the context of intersecting and magnetized 7branes, one may provide a dual description of such system in the more familiar context of magnetized D9-branes, as we show in appendix A.

\subsection{Local F-theory models from intersecting 7-branes}

Following [6-9] (see also [26-34]), one may construct a local F-theory model from a set of 7-branes wrapping a compact divisor $S$ of the threefold base $B$ of an elliptically-fibered Calabi-Yau fourfold. The gauge group $G_{S}$ on such 7-branes is specified by the singularity type of the elliptic fiber on top of the 4-cycle $S$. More precisely, $G_{S}$ depends on the fiber singularity in the bulk of $S$, as such singularity may be enhanced to a higher type on certain complex submanifolds of $S$. In particular, an enhancement in a curve $\Sigma \subset S$ happens whenever $\Sigma=S \cap S^{\prime}$ is the intersection locus of $S$ with another divisor $S^{\prime}$ of $B$ where a different set of 7 -branes is wrapped. We can then associate two other gauge groups $G_{S^{\prime}}$ and $G_{\Sigma}$ to $S^{\prime}$ and $\Sigma$, respectively, and it is easy to see that $G_{S} \times G_{S^{\prime}} \subset G_{\Sigma}$. As in the case of intersecting D7-branes, the intersection locus $\Sigma=S \cap S^{\prime}$ hosts matter fields charged under the gauge group $G_{S} \times G_{S^{\prime}}$. Similarly, an enhancement on a point $p \in S$ happens whenever $p$ is the intersection locus of two or more of these matter curves. Of particular interest for GUT model building are the triple intersections of matter curves, since they give rise to Yukawa couplings between three matter fields charged under $G_{S}$, which is in turn identified with the GUT gauge group.

The dynamics governing the above construction can be encoded in the $8 \mathrm{~d}$ effective action found in [7] which, upon dimensional reduction on the 4-cycle $S$, provides the dynamics of the $4 \mathrm{~d}$ degrees of freedom. ${ }^{3}$ In particular, the Yukawa couplings between $4 \mathrm{~d}$

\footnotetext{
${ }^{3}$ Alternatively, one may derive such dynamics from a 8d SYM Lagrangian [17].
} 
chiral fields arise from the superpotential

$$
W=m_{*}^{4} \int_{S} \operatorname{Tr}(F \wedge \Phi)
$$

where $m_{*}^{4}$ is the F-theory characteristic scale, $F=d A-i A \wedge A$ is the field strength of the gauge vector boson $A$ arising from a stack of 7 -branes, and $\Phi$ is a (2,0)-form on the 4-cycle $S$ describing its transverse geometrical deformations. Locally, we can take both $A$ and $\Phi$ to transform in the adjoint of the non-Abelian gauge group $G_{p} \supset G_{S}$ associated to the enhanced singularity at the Yukawa point $p$. This initial gauge group is broken by the fact that $\Phi$ and $A$ have a non-trivial profile, and so the actual gauge group is the commutant of $H$ in $G_{p}$, with $H$ the subgroup generated by $\langle\Phi\rangle$ and $\langle A\rangle$.

Assuming that $[\langle\Phi\rangle,\langle A\rangle]=0$, we can write $H=H_{\Phi} \times H_{F}$, and consider the effect of $\langle\Phi\rangle$ and $\langle A\rangle$ separately. On one hand, the effect of $\langle\Phi\rangle$ is to describe the system of intersecting divisors considered above, so that $G_{\Phi}=\left[H_{\Phi}, G_{p}\right]=G_{S} \times \prod_{i} G_{i}$, with $G_{i}$ the gauge groups associated to 7-branes wrapping the divisors $S_{i}$ intersecting $S$ on $\Sigma_{i}$. In particular, for a generic point of $S$ the rank of $\langle\Phi\rangle$ is given by $\operatorname{rank}\langle\Phi\rangle=\operatorname{rank} G_{p}-\operatorname{rank} G_{S}$, while it decreases to $\operatorname{rank} G_{p}-\operatorname{rank} G_{\Sigma_{i}}$ on top of the matter curve $\Sigma_{i}$ and vanishes at $p$. On the other hand, the effect of $\langle A\rangle$ is to provide a $4 \mathrm{~d}$ chiral spectrum and to further break the GUT gauge group $G_{S}$ down to the subgroup $\left[H_{F}, G_{S}\right]$, as it is usual in compactifications with magnetized D-branes [4, 35-40]. Hence, one may obtain a 4d MSSM spectrum from the above construction by first engineering the appropriate GUT 4d chiral spectrum via $\langle\Phi\rangle$ and an $\langle A\rangle$ which commutes with $G_{S}$, and then turn on an extra component of $\langle A\rangle$ along the hypercharge generator in order to break $G_{S} \rightarrow G_{M S S M}[8]$.

\subsection{Zero and massive modes at the intersection}

While the above scheme provides a general strategy to construct MSSM-like F-theory models, how close a particular construction is to the MSSM crucially depends on the spectrum of $4 \mathrm{~d}$ zero modes localized at $S$ and of the couplings between them. Again, such information is encoded in the superpotential (2.1) which, together with the D-term for $S$,

$$
D=\int_{S} \omega \wedge F+\frac{1}{2}[\Phi, \bar{\Phi}]
$$

(where $\omega$ stands for the fundamental form of $S$ ) specify the spectrum of $4 \mathrm{~d}$ zero and massive modes as a set of internal wavefunctions along $S$, and the couplings between these $4 \mathrm{~d}$ modes in terms of overlapping integrals of such wavefunctions. ${ }^{4}$

Indeed, variating $A$ and $\Phi$ in the superpotential (2.1), one obtains the F-term equations

$$
\begin{aligned}
\bar{\partial}_{A} \Phi & =0 \\
F^{(0,2)} & =0
\end{aligned}
$$

where $\bar{\partial}_{A}=\left(\partial_{\bar{x}}-i A_{\bar{x}}\right) d \bar{x}+\left(\partial_{\bar{y}}-i A_{\bar{y}}\right) d \bar{y}$ is the anti-holomorphic piece of the covariant derivative operator $D_{A}=\partial_{A}+\bar{\partial}_{A}$ on the 4 -cycle $S$, of local complex coordinates $(x, y)$. In

\footnotetext{
${ }^{4}$ This D-term receives $\alpha^{\prime}$ corrections, and it is also modified by the presence of warping. See [41].
} 
addition, from (2.2) we obtain the D-term equation

$$
\omega \wedge F+\frac{1}{2}[\Phi, \bar{\Phi}]=0
$$

where in this local coordinate system $\omega$ can be described as

$$
\omega=\frac{i}{2}(d x \wedge d \bar{x}+d y \wedge d \bar{y}) .
$$

At the level of the background $\langle A\rangle$ and $\langle\Phi\rangle$, the equations (2.3) and (2.4) reduce to the usual supersymmetry conditions for the 7-brane embedding. As $[\langle\Phi\rangle,\langle A\rangle]=0$, eq. (2.3a) implies that $\left\langle\Phi_{x y}\right\rangle$ is holomorphic on $(x, y)$, consistently with the fact that $S$ and $S_{i}$ are all holomorphic 4-cycles. If in addition $\left\langle\Phi_{x y}\right\rangle$ lives in the Cartan subalgebra of $G_{p}$ then $[\langle\Phi\rangle,\langle\bar{\Phi}\rangle]=0$ and so eqs. (2.3b) and (2.4) imply that $\langle F\rangle$ is a primitive $(1,1)$-form on $S$, similarly to the case of D7-branes in type IIB Calabi-Yau compactifications.

One may, in addition, also obtain the equation of motion for the 7-brane bosonic fluctuations from the above BPS equations. Indeed, by defining

$$
\Phi_{x y}=\left\langle\Phi_{x y}\right\rangle+\varphi_{x y} \quad A_{\bar{m}}=\left\langle A_{\bar{m}}\right\rangle+a_{\bar{m}}
$$

and expanding eqs. (2.3) and (2.4) to first order in the fluctuations $\left(\varphi, a_{\bar{x}}, a_{\bar{y}}\right)$ one obtains

$$
\begin{aligned}
\bar{\partial}_{\langle A\rangle} \varphi+i[\langle\Phi\rangle, a] & =0 \\
\bar{\partial}_{\langle A\rangle} a & =0 \\
\omega \wedge \partial_{\langle A\rangle} a-\frac{1}{2}[\langle\bar{\Phi}\rangle, \varphi] & =0
\end{aligned}
$$

where $a=a_{\bar{x}} d \bar{x}+a_{\bar{y}} d \bar{y}$ and $\varphi=\varphi_{x y} d x \wedge d y$. These are indeed the zero mode equations of motion for the bosonic fluctuations as obtained from the $8 \mathrm{~d}$ action derived in [7], and which pair up with the zero mode fermionic fluctuations in $4 \mathrm{~d} \mathcal{N}=1$ chiral multiplets as $\left(a_{\bar{m}}, \psi_{\bar{m}}\right)$ and $\left(\varphi_{x y}, \chi_{x y}\right)$. The equation of motion for the latter degrees of freedom can be obtained from the part of the $8 \mathrm{~d}$ action bilinear in fermions, and read $[7,15]$

$$
\begin{aligned}
\bar{\partial}_{A} \chi+i[\Phi, \psi]-2 i \sqrt{2} \omega \wedge \partial_{A} \eta & =0 \\
\bar{\partial}_{A} \psi-i \sqrt{2}[\bar{\Phi}, \eta] & =0 \\
\omega \wedge \partial_{A} \psi-\frac{1}{2}[\bar{\Phi}, \chi] & =0
\end{aligned}
$$

where for simplicity we have replaced $\langle\Phi\rangle \rightarrow \Phi$ and $\langle A\rangle \rightarrow A$, and have included the fermionic degree of freedom within the gauge multiplet $\left(A_{\mu}, \eta\right)$. The latter set of equations can be expressed in matrix notation as

$$
\mathbf{D}_{\mathbf{A}} \Psi=0
$$

where

$$
\mathbf{D}_{\mathbf{A}}=\left(\begin{array}{cccc}
0 & D_{x} & D_{y} & D_{z} \\
-D_{x} & 0 & -D_{\bar{z}} & D_{\bar{y}} \\
-D_{y} & D_{\bar{z}} & 0 & -D_{\bar{x}} \\
-D_{z} & -D_{\bar{y}} & D_{\bar{x}} & 0
\end{array}\right) \quad \Psi=\left(\begin{array}{c}
\psi_{\overline{0}} \\
\psi_{\bar{x}} \\
\psi_{\bar{y}} \\
\psi_{\bar{z}}
\end{array}\right) \equiv\left(\begin{array}{c}
-\sqrt{2} \eta \\
\psi_{\bar{x}} \\
\psi_{\bar{y}} \\
\chi_{x y}
\end{array}\right)
$$


where $D_{m}=\partial_{m}-i\left[A_{m}, \cdot\right], m=x, y, z$ is the covariant derivative. In order to define $D_{\bar{z}}$ we are identifying $A_{\bar{z}}=\Phi_{x y}$ and imposing that all fields are $z$-independent, so that $D_{\bar{z}}=-i\left[\Phi_{x y}, \cdot\right]$. As discussed in appendix A, these identifications arise from relating a system of intersecting D7-branes with a system of magnetized D9-branes by T-duality. In such D9-brane picture eq. (2.9) is nothing but the standard Dirac equation for the fermionic zero modes, $\mathbf{D}_{\mathbf{A}}$ being the usual Dirac operator.

Interestingly, the latter point allows to write down the eigenmode equation for the 7brane massive modes in a rather simple way. Indeed, by analogy with the D9-brane picture we have that a fermionic mode of mass $m_{\rho}$ must satisfy

$$
\mathbf{D}_{\mathbf{A}}^{\dagger} \mathbf{D}_{\mathbf{A}} \Psi=\left|m_{\rho}\right|^{2} \Psi
$$

where $\mathbf{D}_{\mathbf{A}}^{\dagger}$ is given by (A.11).

\subsection{A U(3) toy model}

In order to illustrate all the above features of F-theory local model building, one may consider a simple toy model made up of three intersecting D7-branes. In particular, let us consider a $\mathrm{U}(3)$ gauge theory on a four-cycle $S$ of local holomorphic coordinates $(x, y)$, and such that the transverse position field $\Phi$ has the vev

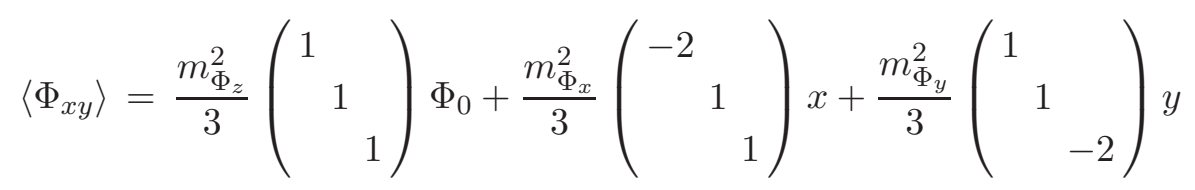

where $m_{\Phi_{x}}, m_{\Phi_{y}}$ and $m_{\Phi_{z}}$ are mass scales introduced so that $\Phi_{x y}$ has the usual dimensions of $L^{-1}$. In the following we will assume that

$$
m_{\Phi_{x}}^{2}=m_{\Phi_{y}}^{2}=m_{\Phi_{z}}^{2} \equiv m_{\Phi}^{2} \quad \text { where } \quad m_{\Phi}^{2}=(2 \pi)^{3 / 2} m_{*}^{2}
$$

leaving the more general case for appendix A. The relation between $m_{*}$ and $m_{\Phi}$ will be motivated in section 3 , around eq. (3.37).

From (2.12) it is easy to see that the initial gauge group is broken as $\mathrm{U}(3) \rightarrow \mathrm{U}(1)^{3}$ by the effect of $\langle\Phi\rangle$ alone, and there is then a rank two enhancement $\mathrm{U}(1) \rightarrow \mathrm{U}(3)$ at the point $p=\left\{(x, y, z)=\left(0,0, \Phi_{0} / 3\right)\right\}$ where the three D7-branes intersect. Such rank two enhancement being generic in the local F-theory GUT setup, one would expect this toy model to capture most of the subtleties involved in computing Yukawa couplings arising from triple intersections of matter curves.

From the geometric viewpoint, the presence of $\langle\Phi\rangle$ indicates that each of the three D7-branes of this model wraps a different four-cycle, algebraically specified by

$$
\begin{aligned}
& S_{\alpha}: 3 z+2 x-y-\Phi_{0}=0 \\
& S_{\beta}: 3 z-x-y-\Phi_{0}=0 \\
& S_{\gamma}: 3 z-x+2 y-\Phi_{0}=0
\end{aligned}
$$


that intersect in the following two-cycles of $S=\left\{z=\Phi_{0} / 3\right\}$

$$
\begin{array}{ll}
S_{\alpha} \cap S_{\beta}: & \Sigma_{a}=\{x=0\} \\
S_{\beta} \cap S_{\gamma}: & \Sigma_{b}=\{y=0\} \\
S_{\gamma} \cap S_{\alpha}: & \Sigma_{c}=\{x=y\}
\end{array}
$$

From the viewpoint of the initial U(3) gauge theory, each of these curves represent a different sector for the fluctuations of a U(3) adjoint field, like the bosonic fields $\left(\phi, a_{\bar{x}}, a_{\bar{y}}\right)$ or the fermionic fields in the vector $\Psi$ in (2.10). In particular, left-handed $4 \mathrm{~d}$ chiral fermions in the bifundamental will arise from U(3) off-diagonal fluctuations of $\Psi$, that we label as

$$
\psi_{\bar{m}}=\left(\begin{array}{ccc}
0 & a_{\bar{m}}^{+} & c_{\bar{m}}^{-} \\
a_{\bar{m}}^{-} & 0 & b_{\bar{m}}^{+} \\
c_{\bar{m}}^{+} & b_{\bar{m}}^{-} & 0
\end{array}\right) \quad \bar{m}=\overline{0}, \bar{x}, \bar{y}, \bar{z}
$$

while their CPT conjugates will be contained in the off-diagonal entries of $\psi_{m}$.

As mentioned above, one extra ingredient necessary to obtain a $4 \mathrm{~d}$ chiral model is the presence of a non-trivial background worldvolume flux $\langle F\rangle$, usually chosen so that $\left[\left\langle\Phi_{x y}\right\rangle,\langle F\rangle\right]=0$. In our $\mathrm{U}(3)$ model, a convenient choice is given by

$$
\langle F\rangle=i\left(M_{x} d x \wedge d \bar{x}+M_{y} d y \wedge d \bar{y}\right) \frac{1}{3}\left(\begin{array}{ccc}
1 & & \\
& -2 & \\
& & 1
\end{array}\right)
$$

so that the background D-term equation (2.4) is satisfied by imposing $M_{y}+M_{x}=0$ pointwise. For simplicity, in the following we will assume that $M_{x}$ and $M_{y}$ are constant but otherwise arbitrary, see [15-17] for the more general case.

In order to derive the chiral spectrum wavefunctions of this toy model let us consider eq. (2.11). In general we have that

$$
\mathbf{D}_{\mathbf{A}}^{\dagger} \mathbf{D}_{\mathbf{A}}=-\Delta \mathbb{I}_{4}-i\left(\begin{array}{cccc}
\sigma_{+++} & 0 & 0 & 0 \\
0 & \sigma_{+--} & F_{y \bar{x}} & F_{z \bar{x}} \\
0 & F_{x \bar{y}} & \sigma_{-+-} & F_{z \bar{y}} \\
0 & F_{x \bar{z}} & F_{y \bar{z}} & \sigma_{--+}
\end{array}\right)
$$

where we have defined $F_{n \bar{z}} \equiv D_{n} \Phi_{x y}$ and $^{5}$

$$
\begin{aligned}
\Delta & =\left\{D_{x}, D_{\bar{x}}\right\}+\left\{D_{y}, D_{\bar{y}}\right\}+\left\{D_{z}, D_{\bar{z}}\right\} \\
\sigma_{\epsilon_{x} \epsilon_{y} \epsilon_{z}} & =\frac{1}{2}\left(\epsilon_{x} F_{x \bar{x}}+\epsilon_{y} F_{y \bar{y}}+\epsilon_{z} F_{z \bar{z}}\right)
\end{aligned}
$$

Finally, $F_{n \bar{m}} \sim\left[F_{n \bar{m}}, \cdot\right]$ acts in the adjoint on the U(3) gauge indices of $\Psi$, which implies that the worldvolume fluxes $F_{n \bar{m}}$ are felt differently by each matter curve. Indeed, for the

\footnotetext{
${ }^{5}$ In our conventions the anticommutator is given by $\{A, B\} \equiv \frac{1}{2}(A B+B A)$.
} 
$a^{ \pm}$sector in (2.16) we have

$$
\mathbf{D}_{\mathbf{A}^{\dagger}}^{\dagger} \mathbf{D}_{\mathbf{A}}=-\left(\Delta_{a^{ \pm}} \pm M_{x y}\right) \mathbb{I}_{4} \pm\left(\begin{array}{cccc}
2 M_{x y} & 0 & 0 & 0 \\
0 & M_{x} & 0 & -i m_{\Phi}^{2} \\
0 & 0 & M_{y} & 0 \\
0 & i m_{\Phi}^{2} & 0 & 0
\end{array}\right)
$$

where $M_{x y} \equiv \frac{1}{2}\left(M_{x}+M_{y}\right)$. For the $b^{ \pm}$sector we have instead

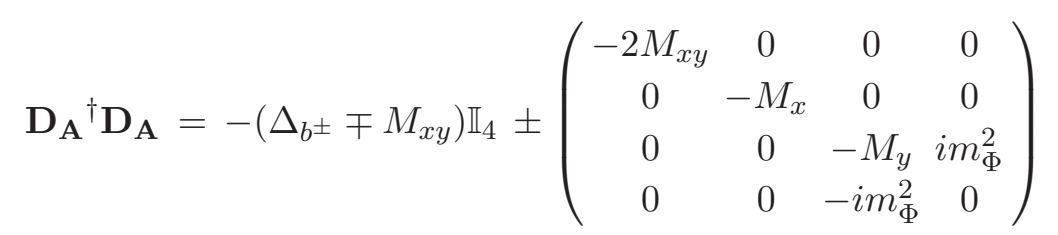

and, finally, for the $c^{ \pm}$sector we have

$$
\mathbf{D}_{\mathbf{A}}^{\dagger} \mathbf{D}_{\mathbf{A}}=-\Delta_{c^{ \pm}} \mathbb{I}_{4} \pm m_{\Phi}^{2}\left(\begin{array}{cccc}
0 & 0 & 0 & 0 \\
0 & 0 & 0 & i \\
0 & 0 & 0 & -i \\
0 & -i & i & 0
\end{array}\right)
$$

Given these expressions and the fact that $M_{x}, M_{y}$ and $m_{\Phi}$ are constant it is easy to find the spectrum of eigenvectors of $\mathbf{D}_{\mathbf{A}}{ }^{\dagger} \mathbf{D}_{\mathbf{A}}$ in terms of the eigenfunctions $-\Delta \psi_{\rho}=\rho^{2} \psi_{\rho}$ of the Laplacian. Indeed, in the case of the sector $a^{ \pm}$we find that the eigenvalues and eigenvectors of the squared Dirac operator are given by

$$
\begin{array}{ll}
\left|m_{\rho}\right|^{2}=\rho^{2} \pm M_{x y}, \Psi=\left(\begin{array}{c}
1 \\
0 \\
0 \\
0
\end{array}\right) \psi_{\rho} ; & \left|m_{\rho}\right|^{2}=\rho^{2} \pm\left(M_{y}-M_{x y}\right), \Psi=\left(\begin{array}{c}
0 \\
0 \\
1 \\
0
\end{array}\right) \psi_{\rho} \\
\left|m_{\rho}\right|^{2}=\rho^{2} \pm\left(\lambda_{a}^{+}-M_{x y}\right), \Psi=\left(\begin{array}{c}
0 \\
\frac{\lambda_{a}^{+}}{m_{\Phi}^{2}} \\
0 \\
i
\end{array}\right) \psi_{\rho} ; \quad\left|m_{\rho}\right|^{2}=\rho^{2} \pm\left(\lambda_{a}^{-}-M_{x y}\right), \Psi=\left(\begin{array}{c}
0 \\
\frac{\lambda_{a}^{-}}{m_{\Phi}^{2}} \\
0 \\
i
\end{array}\right) \psi_{\rho}
\end{array}
$$

where

$$
\lambda_{a}^{ \pm}=\frac{M_{x}}{2} \pm \sqrt{\left(\frac{M_{x}}{2}\right)^{2}+m_{\Phi}^{4}}
$$

The precise expression for $\psi_{\rho}$ does in principle depend on which sector we consider, as the Laplacian (2.19) depends non-trivially on the gauge potential $A$, which acts differently on $a^{ \pm}$. By taking a real gauge

$$
\langle A\rangle^{\text {real }}=\frac{i}{2}\left[M_{x}(x d \bar{x}-\bar{x} d x)+M_{y}(y d \bar{y}-\bar{y} d y)\right] \frac{1}{3}\left(\begin{array}{ccc}
1 & & \\
& -2 & \\
& & 1
\end{array}\right)
$$


is easy to see that the modes satisfying the zero mode equation (2.9) for the sectors $a^{ \pm}$are given by

$$
\Psi_{0, a^{ \pm}}^{\text {real }}=\left(\begin{array}{c}
0 \\
\frac{\lambda_{a}^{\mp}}{m_{\Phi}^{2}} \\
0 \\
i
\end{array}\right) e^{-\sqrt{\left(\frac{M x}{2}\right)^{2}+m_{\Phi}^{4}}|x|^{2}} e^{\mp \frac{M y}{2}|y|^{2}} f_{a^{ \pm}}(y)
$$

with $f_{a^{ \pm}}$an arbitrary holomorphic function on the intersection coordinate $y$. It is then easy to see that the zero modes on the $a^{ \pm}$sector will only converge for $\pm M_{y}>0$, reproducing the usual behavior of magnetized D-brane systems [4].

For our purposes, however, it is more convenient to express the zero mode wavefunctions in the holomorphic gauge introduced in [15], in which only the holomorphic components of $\langle A\rangle$ are non-vanishing. In the model at hand, such gauge reads

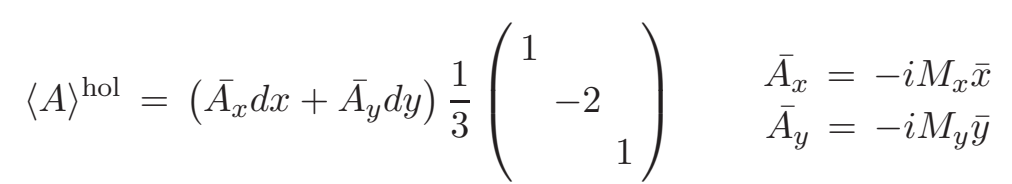

and it is easy to see that the corresponding zero mode wavefunctions are given by

$$
\Psi_{0, a^{ \pm}}^{\mathrm{hol}}=\left(\begin{array}{c}
0 \\
-i \frac{\lambda_{a}^{\mp}}{m_{\Phi}^{2}} \\
0 \\
1
\end{array}\right) \psi_{0, a^{ \pm}}, \quad \psi_{0, a^{ \pm}}=e^{ \pm \lambda_{a}^{\mp}|x|^{2}} f_{a^{ \pm}}(y)
$$

In fact, from the Laplace eigenfunction $\psi_{0, a^{ \pm}}$one may easily construct all the other eigenfunctions of the Laplace operator $\Delta_{a^{ \pm}}$, and so the full spectrum of massive modes in this sector. Indeed, following the discussion in appendix A we have that all the eigenfunctions of $\Delta_{a^{ \pm}}$are of the form

$$
\psi_{m n l, a^{ \pm}}=\left(\mathcal{D}_{x}\right)^{m}\left(\mathcal{D}_{y}\right)^{n}\left(\mathcal{D}_{\bar{z}}\right)^{l} \psi_{0, a^{ \pm}}
$$

with the operators $\mathcal{D}_{m}$ defined in (A.23).

A similar discussion can be carried out for the sectors $b^{ \pm}$and $c^{ \pm}$. Leaving the details for appendix A, we obtain that the zero mode wavefunctions in the holomorphic gauge for these sectors are

$$
\Psi_{0, b^{ \pm}}^{\mathrm{hol}}=\left(\begin{array}{c}
0 \\
0 \\
i \frac{\lambda_{b}^{\mp}}{m_{\Phi}^{2}} \\
1
\end{array}\right) \psi_{0, b^{ \pm}}, \quad \psi_{0, b^{ \pm}}=e^{ \pm \lambda_{b}^{\mp}|y|^{2}} f_{b^{ \pm}}(x)
$$

and

$$
\Psi_{0, c^{ \pm}}^{\mathrm{hol}}=\left(\begin{array}{c}
0 \\
i \frac{\lambda_{c}}{m_{\Phi}^{2}} \\
-i \frac{\lambda_{c}}{m_{\Phi}^{2}} \\
1
\end{array}\right) \psi_{0, c^{ \pm}}, \quad \psi_{0, c^{ \pm}}=\gamma_{c} m_{*} e^{\lambda_{c}|x-y|^{2}}
$$


where

$$
\lambda_{b}^{ \pm}=-\frac{M_{y}}{2} \pm \sqrt{\left(\frac{M_{y}}{2}\right)^{2}+m_{\Phi}^{4}} \quad ; \quad \lambda_{c}=-\frac{m_{\Phi}^{2}}{\sqrt{2}}
$$

and with similar expressions to (2.30) for their massive replicas. In the following we will consider our zero and massive mode wavefunctions in the holomorphic gauge, avoiding any superscript that indicates so. We will, in addition, assume that $M_{x}<0<M_{y}$, so that the sectors of interest for computing zero mode Yukawa couplings are $a^{+}, b^{+}$and $c^{+}$. Finally, in (2.32) we have introduced a normalization factor to be fixed later.

\subsection{Yukawa couplings}

The superpotential (2.1) gives rise to Yukawa couplings among the 4-dimensional charged fields since it includes the trilinear term

$$
W_{\text {Yuk }}=-i m_{*}^{4} \int_{S} \operatorname{Tr}(A \wedge A \wedge \Phi)
$$

that induces Yukawa couplings between the zero (and massive) modes of $A$ and $\Phi$. In particular, in the above setup where charged massless matter resides at curves where 7branes intersect, the Yukawa couplings $Y_{a b c}^{i j k}$ are generated at the intersection of three matter curves $\Sigma_{a}, \Sigma_{b}$ and $\Sigma_{c}$, whose zero modes are respectively indexed by $i, j, k$.

To describe the Yukawa couplings it is useful to define the vector

$$
\vec{\psi}=\left(\begin{array}{c}
\psi_{\bar{x}} \\
\psi_{\bar{y}} \\
\chi_{x y}
\end{array}\right)=\vec{\psi}_{\alpha} \mathfrak{t}_{\alpha}
$$

which is a subvector of $\Psi$ in (2.10). Here $\mathfrak{t}_{\alpha}$ is a generator of the Lie algebra $\mathfrak{g}_{p}$ of the enhanced group $G_{p}$ at the Yukawa point $p$, with the normalization $\operatorname{Tr} \mathfrak{t}_{\alpha} \mathfrak{t}_{\beta}^{\dagger}=\delta_{\alpha \beta}$. More precisely, $\mathfrak{t}_{\alpha}$ is the generator associated to a root $\alpha$ of $\mathfrak{g}_{p}$, which in turn corresponds to a matter curve $\Sigma_{\alpha}$ going through that point (see below for an example). The components of $\vec{\psi}_{\alpha}$ are scalar wavefunctions describing localized modes at such curve, and in particular its zero modes. As each curve may host several zero modes, we will label each zero mode vector by $\vec{\psi}_{\alpha}^{i}, i$ being the family index.

Recall that the $\psi_{\bar{m}}$ are the superpartners of the fluctuations $a_{\bar{m}}$ of $A$, whereas $\chi_{x y}$ belongs to the same multiplet as the fluctuations $\varphi_{x y}$ of $\Phi$. Notice that, as implicit in (2.34), the fermion $\eta$ in the gauge multiplet does not contribute to the Yukawa couplings, which is why such degree of freedom does not enter in the definition of $\vec{\psi}$. In fact, as noticed in $[7,15]$ and shown explicitly in the $\mathrm{U}(3)$ model above, matter curve zero modes do not have a non-trivial component along $\eta$. As shown in appendix $\mathrm{B}$, the same applies to the zero modes that arise in the presence of a non-perturbative deformation.

Inserting the zero modes in $W_{\text {Yuk }}$ gives the couplings

$$
Y_{a b c}^{i j k}=m_{*} f_{a b c} \int_{S} \operatorname{det}\left(\vec{\psi}_{a}^{i}, \vec{\psi}_{b}^{j}, \vec{\psi}_{c}^{k}\right) \operatorname{dvol}_{S}
$$

where $f_{a b c}=-i \operatorname{Tr}\left(\left[\mathfrak{t}_{a}, \mathfrak{t}_{b}\right] \mathfrak{t}_{c}\right)$ and the integration measure is given by $\operatorname{dvol}_{S}=2 \omega^{2}=$ $\mathrm{d} x \wedge \mathrm{d} y \wedge \mathrm{d} \bar{x} \wedge \mathrm{d} \bar{y}$. 
In the following we will compute the $Y_{a b c}^{i j k}$ for the U(3) toy model. Since the couplings are gauge invariant we can conveniently work in the holomorphic gauge in which the zero modes take a simpler form. Turning on 7-brane fluxes $M_{x}<0<M_{y}$, there will be normalizable zero modes in the $a^{+}$and $b^{+}$sectors, which couple to those in the $c^{+}$sector. Indeed, given the $\mathrm{U}(3)$ structure displayed in (2.16) we see that

$$
\mathfrak{t}_{a^{+}}=\left(\begin{array}{ccc}
0 & 1 & 0 \\
0 & 0 & 0 \\
0 & 0 & 0
\end{array}\right) \quad \mathfrak{t}_{b^{+}}=\left(\begin{array}{ccc}
0 & 0 & 0 \\
0 & 0 & 1 \\
0 & 0 & 0
\end{array}\right) \quad \mathfrak{t}_{c^{+}}=\left(\begin{array}{lll}
0 & 0 & 0 \\
0 & 0 & 0 \\
1 & 0 & 0
\end{array}\right)
$$

and so $\operatorname{Tr}\left(\left[\mathfrak{t}_{a^{+}}, \mathfrak{t}_{b^{+}}\right] \mathfrak{t}_{c^{+}}\right)=1$. We will take the Higgs to arise from the non-chiral sector which is curve $\Sigma_{c}$, while the chiral families will arise from the curves $\Sigma_{a}$ and $\Sigma_{b}$, and will be indexed by $i$ and $j$ respectively. Finally, the Yukawa couplings will be denoted by $Y^{i j}$.

From the results of subsection 2.3 and appendix A we see that the vectors (2.35) for the $\mathrm{U}(3)$ model read

$$
\vec{\psi}_{a^{+}}^{i}=\left(\begin{array}{c}
-\frac{i \lambda_{a}}{m_{\Phi}^{2}} \\
0 \\
1
\end{array}\right) \chi_{a^{+}}^{i} \quad ; \quad \vec{\psi}_{b^{+}}^{j}=\left(\begin{array}{c}
0 \\
\frac{i \lambda_{b}}{m_{\Phi}^{2}} \\
1
\end{array}\right) \chi_{b^{+}}^{j} \quad ; \quad \vec{\psi}_{c^{+}}=\left(\begin{array}{c}
\frac{i \lambda_{c}}{m_{\Phi}^{2}} \\
-\frac{i \lambda_{c}}{m_{\Phi}^{2}} \\
1
\end{array}\right) \chi_{c^{+}}
$$

where $\lambda_{a}=\lambda_{a}^{-}, \lambda_{b}=\lambda_{b}^{-}$and $\lambda_{c}$ are defined in (2.25) and (2.33), and the scalar wavefunctions $\chi$ are given by

$$
\chi_{a^{+}}^{i}=e^{\lambda_{a}|x|^{2}} f_{i}(y) \quad ; \quad \chi_{b^{+}}^{j}=e^{\lambda_{b}|y|^{2}} g_{j}(x) \quad ; \quad \chi_{c^{+}}=\gamma_{c} m_{*} e^{\lambda_{c}|x-y|^{2}}
$$

For the different zero modes we will follow [13] and take a basis in which $f_{i}(y)=\gamma_{a i} m_{*}^{4-i} y^{3-i}$ and $g_{j}(x)=\gamma_{b j} m_{*}^{4-j} x^{3-j}, i, j=1,2,3$, mimicking the physical case with three families of quarks and leptons. The normalization factors $\gamma_{a i}$ and $\gamma_{b j}$ will be fixed later.

Substituting in (2.36) readily gives the couplings

$$
Y^{i j}=-i \gamma_{c} \frac{m_{*}^{2}}{m_{\Phi}^{4}}\left[\lambda_{a} \lambda_{b}+\lambda_{c}\left(\lambda_{a}+\lambda_{b}\right)\right] \int_{S} e^{\lambda_{a}|x|^{2}+\lambda_{b}|y|^{2}+\lambda_{c}|x-y|^{2}} f_{i}(y) g_{j}(x) \operatorname{dvol}_{S}
$$

Notice that the exponential and the measure of the integral are invariant under the diagonal $\mathrm{U}(1)$ rotation $x \rightarrow e^{i \alpha} x$ and $y \rightarrow e^{i \alpha} y$. Therefore, the only non-vanishing coupling is $Y^{33}$ because $f_{3}$ and $g_{3}$ are constant. Even though we are working with a local model for $S$, to evaluate the integral in (2.40) we extend $|x|$ and $|y|$ to infinite radius. This is justified because the exponentials are localized on the matter curves and the error due to extending the Gaussian integrals is negligible. An elementary calculation then gives the exact result

$$
\int_{S} e^{\lambda_{a}|x|^{2}+\lambda_{b}|y|^{2}+\lambda_{c}|x-y|^{2}} \operatorname{dvol}_{S}=\pi^{2}\left[\lambda_{a} \lambda_{b}+\lambda_{c}\left(\lambda_{a}+\lambda_{b}\right)\right]^{-1}
$$

Hence, the only non-vanishing Yukawa is given by

$$
Y^{33}=-i \pi^{2} \frac{m_{*}^{4}}{m_{\Phi}^{4}} \gamma_{a 3} \gamma_{b 3} \gamma_{c}
$$


With normalization $\gamma_{a 3}=\gamma_{b 3}=\gamma_{c}=1$, the coupling is completely independent of the worldvolume flux. Moreover, this result holds without imposing the D-term BPS condition (2.4) on the background, as already noticed in the addendum of [15]. In [16], Yukawa independence on 7-brane worldvolume fluxes was derived from an exact residue formula.

\section{$3 \quad$ Non-perturbative effects on intersecting 7-branes}

As shown above for the $\mathrm{U}(3)$ model and more generally in [16], Yukawa couplings do not depend on 7-brane worldvolume fluxes, ${ }^{6}$ and this result has drastic consequences from the viewpoint of the fermion mass matrices. Namely, if all Yukawa couplings arise from a single triple intersection, the Yukawa matrices derived from (2.36) will have rank one for any choice of worldvolume flux, and so only one family of quarks and leptons will receive a non-trivial mass in such F-theory construction [16]. While this is a promising starting point to generate the observed hierarchical structure of fermion masses, one still needs an extra ingredient beyond the intersecting 7-brane setup that slightly perturbs the Yukawas away from this rank-one result.

As pointed out in [23], such extra contribution to the Yukawa couplings will in general arise from non-perturbative effects on a 7-brane far away from the GUT 4-cycle $S$. Indeed, if we consider a distant 7-brane whose $4 \mathrm{~d}$ gauge theory undergoes a gaugino condensation, then a non-perturbative superpotential will be generated for the GUT 7-brane fields, perturbing the previous tree-level superpotential. In particular, there will a non-trivial contribution to the tree-level Yukawa couplings, so that we will instead have

$$
Y_{\text {total }}^{i j k}=Y_{\text {tree }}^{i j k}+Y_{\mathrm{np}}^{i j k}
$$

where $Y_{\text {tree }}^{i j k}$ corresponds to the tree-level contribution (2.36), while $Y_{\mathrm{np}}^{i j k}$ stands for the new set of Yukawa couplings that arise at the non-perturbative level. In general $\left|Y_{\mathrm{np}}^{i j k}\right| \ll\left|Y_{\text {tree }}^{i j k}\right|$, and the non-perturbative couplings will provide a slight deviation from the tree-level rankone result. Finally, the same scenario applies if instead of a gaugino condensate on a 7-brane one considers the effect of an Euclidean 3-brane on the same 4-cycle. ${ }^{7}$

Remarkably, as shown in [23] such non-perturbative contribution can be computed rather precisely in the case of intersecting 7-branes. In fact, there is not only one, but rather several approaches that one may use in order to compute (3.1). The purpose of this section is to introduce each one of these approaches separately and show that, at least in the approximation scheme that we will discuss, all lead to the same result.

The first and more conventional approach consists in computing the non-perturbative effect at the level of the $4 \mathrm{~d}$ effective action, in terms of a non-perturbative superpotential $W_{\text {np }}$ generated for the $4 \mathrm{~d}$ massless and massive fields of the GUT 7-brane. The second approach consists in treating such non-perturbative superpotential as a $8 \mathrm{~d}$ deformation of (2.1). Notice that, before dimensional reduction to $4 \mathrm{~d}$, the superpotential (2.1) can be understood as a functional of the $8 \mathrm{~d}$ fields $A$ and $\Phi$. In this $8 \mathrm{~d}$ approach, the nonperturbative effect is also understood as a functional $W_{\beta}$ of $(A, \Phi)$, that adds up to the

\footnotetext{
${ }^{6}$ That is, provided that the latter satisfy the F-term BPS conditions (2.3b) at the level of background.

${ }^{7}$ See [5] for an earlier proposal along this lines for the rank-one intersecting D6-brane model of [42].
} 
functional (2.1) and modifies the wavefunction and Yukawa computation of section 2. Finally, the third approach is a variant of the $8 \mathrm{~d}$ approach, in the sense that the analysis is also performed at the level of $8 \mathrm{~d}$ fields $(\hat{A}, \hat{\Phi})$. The non-perturbative effect, however, is now seen as a non-commutative deformation of the functional (2.1), in the sense of [16].

Besides describing these different approaches, in this section we will discuss how the (commutative) 8d approach reproduces the results of the more standard effective $4 \mathrm{~d}$ approach. The matching between commutative and non-commutative $8 \mathrm{~d}$ approaches will be postponed to section 4 and appendix C. In particular, in subsection 4.2 we will provide a dictionary between those wavefunctions computed in the non-commutative $8 \mathrm{~d}$ formalism (see section 5) and those computed in the commutative 8d approach (see appendix B). As all these approaches lead to the same physics, the reader who is just interested in the final result for the Yukawa couplings may safely skip section 4 and proceed to section 5, where such Yukawas are computed explicitly for the U(3) model.

\section{$3.14 d$ approach}

In general, when computing non-perturbative effects in a string compactification, one does so at the level of the $4 \mathrm{~d}$ effective theory. In particular, for the 7-brane setup considered above one would first compute the gauge kinetic function $f_{7_{\mathrm{np}}}$ of the stack of $n 7$-branes undergoing a gaugino condensation, and then use the standard $4 \mathrm{~d}$ expression

$$
W_{\mathrm{np}}^{4 \mathrm{~d}}=\mu^{3} e^{-f_{7 \mathrm{np}} / n}
$$

to compute the gaugino condensate contribution to the $4 \mathrm{~d}$ effective superpotential. Here $\mu \sim m_{*}$ is the UV scale at which $f_{7 \mathrm{np}}$ is defined. From the IR viewpoint, $f_{7 \mathrm{np}}$ should be understood as a holomorphic function of the $4 \mathrm{~d}$ chiral multiplets of the theory, which arise either from the bulk or from the 7-brane sectors of the compactification. More precisely, such 7-brane kinetic function is of the form

$$
f_{7_{\mathrm{np}}}=T_{\mathrm{np}}+f_{7_{\mathrm{np}}}^{1-\text { loop }}\left(B_{i}, C_{j}\right)
$$

where the first contribution amounts to the gauge kinetic function $f_{7_{\mathrm{np}}}$ computed at treelevel, and is given by the complexified Kähler modulus $T_{\mathrm{np}}=\operatorname{Vol}\left(S_{\mathrm{np}}\right)+i \int_{S_{\mathrm{np}}} C_{4}$ corresponding to the 4-cycle $S_{\mathrm{np}}$ wrapped by the gaugino condensing 7-branes. The second contribution arises from threshold effects, and is given by a holomorphic function $f_{7_{\mathrm{np}}}^{1-\text { loop }}$ of the bulk/closed string fields $\left\{B_{i}\right\}$, and of the $4 \mathrm{~d}$ fields $\left\{C_{j}\right\}$ arising from the remaining 7-brane sectors of the compactification. The latter set of fields can be divided as $\left\{C_{j}\right\}=\left\{L_{j}, H_{k}, X_{l}\right\}$ where $\left\{L_{j}\right\}$ are massless and $\left\{H_{k}\right\}$ massive fields arising from 7-brane intersections and $\left\{X_{l}\right\}$ massive fields spread out along the whole 7-brane worldvolume. ${ }^{8}$ The massive fields $\left\{H_{k}\right\}$ and $\left\{X_{l}\right\}$ are usually integrated out and thus not considered in the expression for $f_{7_{\text {np }}}^{1-\text { loop }}$, but we will see that including them is crucial for our analysis.

From this $4 \mathrm{~d}$ viewpoint, the main problem is to find $f_{7_{\mathrm{np}}}^{1-\mathrm{loop}}$ as a function of massless and massive $4 \mathrm{~d}$ fields. This is however implicit in the expression

$$
f_{7_{\mathrm{np}}}^{1-\text { loop }}=-n \log \mathcal{A}-\frac{1}{8 \pi^{2}} \int_{S} \mathrm{~S} \operatorname{Tr}(\log h F \wedge F)
$$

\footnotetext{
${ }^{8}$ We are assuming that no chiral or massless fields arise from this sector.
} 
derived in [23]. Here $h$ is the divisor function of the 4-cycle $S_{\mathrm{np}}=\{h=0\}$ where the non-perturbative effect is taking place, and $\mathcal{A}$ is a function of the bulk/closed string fields $B_{i}$ which will not play any role in the following discussion and can be replaced by their vev $\left\langle B_{i}\right\rangle$. While $\log h$ is a scalar bulk quantity, when plugged into the expression (3.4) one should follow the prescription of [43] and consider its non-Abelian pull-back into $S$. That is

$$
\log h=\left.\log h\right|_{S}+m_{\Phi}^{-2} \Phi^{m}\left[\mathcal{L}_{m} \log h\right]_{S}+m_{\Phi}^{-4} \Phi^{m} \Phi^{n}\left[\mathcal{L}_{m} \mathcal{L}_{n} \log h\right]_{S}+\ldots
$$

with $\mathcal{L}_{m} \equiv \mathcal{L}_{X_{m}}$ the Lie derivative along a vector $X_{m}$ transverse to $S$. Since $h$ is holomorphic so will be $X_{m}$ and so, in the local coordinate system used above, we should take $X_{m}=z$. Also, if as we assume that $S_{\mathrm{np}}$ is distant from our GUT 4-cycle $S$, and in particular that they do not intersect, then $\left.h\right|_{S}$ will be a holomorphic function of $S$ with no zeroes or poles, hence a constant. This implies that

$$
-f_{7_{\mathrm{np}}}^{1-\mathrm{loop}}=n \log \mathcal{A}+\left.N_{\mathrm{D} 3} \log h\right|_{S}+\frac{m_{\Phi}^{-2}}{8 \pi^{2}} \int_{S}\left[\partial_{z} \log h\right]_{S} \operatorname{Tr}\left(\Phi^{z} F \wedge F\right)+\ldots
$$

where $N_{D 3}=\left(8 \pi^{2}\right)^{-1} \int_{S} \operatorname{Tr}(F \wedge F) \in \mathbb{N}$ stands for the D3-brane charge induced by the presence of $F$ and we are not displaying higher orders in $m_{\Phi}^{-2}$. Clearly, the dependence of $f_{7_{\text {np }}}^{1-\text { loop }}$ on the 7 -brane fields $\left\{C_{j}\right\}$ arises only from the third term of the rhs of (3.6), and is still implicit in the integral over the GUT 4-cycle $S$. In order to extract such dependence one must insert the internal wavefunctions for the fields $\left\{C_{j}\right\}$ in the term $\operatorname{Tr}\left(\Phi^{z} F \wedge F\right)$, and then perform the integral over $S$ in order to obtain the different $4 \mathrm{~d}$ couplings.

Once done so, it is straightforward to compute the non-perturbative contribution to the full $4 \mathrm{~d}$ superpotential. Indeed, inserting (3.6) into (3.2) we obtain

$$
\begin{aligned}
W_{\mathrm{np}}^{4 \mathrm{~d}} & =\mu^{3}\left(\left.\mathcal{A} e^{-T_{\mathrm{np}} / n} h^{N_{\mathrm{D} 3} / n}\right|_{S}\right) \exp \left[\frac{\left(m_{\Phi}^{2} n\right)^{-1}}{8 \pi^{2}} \int_{S}\left[\partial_{z} \log h\right]_{S} \operatorname{Tr}\left(\Phi^{z} F \wedge F\right)+\ldots\right] \\
& =\mu^{3} \epsilon\left(1+\frac{\left(m_{\Phi}^{2} n\right)^{-1}}{8 \pi^{2}} \int_{S}\left[\partial_{z} \log h\right]_{S} \operatorname{Tr}\left(\Phi^{z} F \wedge F\right)+\ldots\right)
\end{aligned}
$$

where we have defined $\epsilon=\left.\mathcal{A} e^{-T_{\mathrm{np}} / n} h\right|_{S} ^{N_{\mathrm{D} 3} / n}$. Upon further defining $\theta=\left.\frac{\mu^{3} / 4 \pi^{2} n}{m_{*}^{4} m_{\Phi}^{2}} \partial_{z} \log h\right|_{S}$ and up to a constant term we have

$$
W_{\mathrm{np}}^{4 \mathrm{~d}}=m_{*}^{4} \frac{\epsilon}{2} \int_{S} \theta \operatorname{Tr}\left(\Phi_{x y} F \wedge F\right)
$$

where we have identified $\Phi_{x y}=\Phi^{z}$ (see next subsection). We can then approximate the total $4 \mathrm{~d}$ superpotential by

$$
W_{\text {total }}^{4 \mathrm{~d}}=W_{\text {tree }}^{4 \mathrm{~d}}+W_{\mathrm{np}}^{4 \mathrm{~d}}=m_{*}^{4}\left[\int_{S} \operatorname{Tr}\left(\Phi_{x y} F\right) \wedge d x \wedge d y+\frac{\epsilon}{2} \int_{S} \theta \operatorname{Tr}\left(\Phi_{x y} F \wedge F\right)\right]
$$

Notice that in this approach the total $4 \mathrm{~d}$ superpotential is obtained by inserting the zero mode wavefunctions computed at tree level (i.e., the ones of section 2) into (3.9) and then performing the appropriate integral. That is, we are dimensionally reducing (3.9) with tree level wavefunctions and background values in order to obtain new $4 \mathrm{~d}$ couplings 
generated non-perturbatively, and from there performing a $4 \mathrm{~d}$ analysis. This is in contrast with the $8 \mathrm{~d}$ philosophy applied in the next subsection, where new internal wavefunctions need to be computed from the very beginning.

Following the $4 \mathrm{~d}$ approach, notice that the dimensional reduction of (2.1) can be written as

$$
W_{\text {tree }}^{4 \mathrm{~d}}=\sum_{i j} \mu^{i j} C_{i} C_{j}+\sum_{i j k} h^{i j k} C_{i} C_{j} C_{k}
$$

where

$$
\begin{aligned}
\mu^{i j} & =m_{*}^{2} \int_{S} \operatorname{Tr}\left(\vec{\psi}^{i} \cdot \bar{\partial}_{\mathbf{A}} \cdot \vec{\psi}^{j}\right) \operatorname{dvol}_{S} \\
h^{i j k} & =m_{*} f_{a b c} \int_{S} \operatorname{det}\left(\vec{\psi}_{a}^{i}, \vec{\psi}_{b}^{j}, \vec{\psi}_{c}^{k}\right) \operatorname{dvol}_{S}
\end{aligned}
$$

Here the vectors $\vec{\psi}$ are defined as in (2.35), and the operator $\bar{\partial}_{\mathbf{A}}$ is the corresponding submatrix of the operator $\mathbf{D}_{\mathbf{A}}$ in (2.10), namely

$$
\bar{\partial}_{\mathbf{A}}=\left(\begin{array}{ccc}
0 & -D_{\bar{z}} & D_{\bar{y}} \\
D_{\bar{z}} & 0 & -D_{\bar{x}} \\
-D_{\bar{y}} & D_{\bar{x}} & 0
\end{array}\right)
$$

Also, in (3.11b) - stands for the usual multiplication of such vectors and matrices. In this sense, it is understood that in (3.11a) $\vec{\psi}$ is given by (2.35) when placed at the right of $\cdot$ and by $\vec{\psi}=\left(\psi_{\bar{x}}, \psi_{\bar{y}}, \chi_{x y}\right)$ when placed at its left. Finally, recall that each of the components of $\vec{\psi}$ is a matrix itself, and that for eigenmodes localized at the matter curve $\Sigma_{\alpha}$ we can write $\vec{\psi}=\vec{\psi}_{\alpha} \mathfrak{t}_{\alpha}$, with $\mathfrak{t}_{\alpha}$ a generator of the enhanced group $G_{\Sigma_{\alpha}}$.

Focusing on such matter curve $\alpha$, from eq. (A.7) we deduce that the wavefunctions there localized must satisfy the equation

$$
i \bar{\partial}_{\mathbf{A}} \vec{\psi}_{H_{k}^{ \pm}}=m_{k} \vec{\psi}_{H_{k}^{\mp}}^{\dagger}
$$

where $H_{k}^{\alpha^{+}}$are the replicas of mass $m_{k}$ of the left-handed chiral multiplets $L_{j}^{\alpha^{+}}, H_{k}^{\alpha^{-}}$the massive chiral multiplet transforming in the conjugate gauge representation and $\vec{\psi}_{H_{k}^{ \pm}}$their corresponding wavefunctions. Finally, $\vec{\psi}^{\dagger}$ is defined as

$$
\vec{\psi}^{\dagger}=\left(\begin{array}{c}
\psi_{\bar{x}}^{\dagger} \\
\psi_{\bar{y}}^{\dagger} \\
\chi_{x y}^{\dagger}
\end{array}\right)
$$

A direct consequence of (3.13) is that, by normalizing our wavefunctions so that

$$
\begin{aligned}
\left\langle\vec{\psi}_{H_{k}^{ \pm}} \mid \vec{\psi}_{H_{l}^{ \pm}}\right\rangle & =m_{*}^{2} \int_{S} \operatorname{Tr}\left(\vec{\psi}_{H_{k}^{ \pm}} \cdot \vec{\psi}_{H_{l}^{ \pm}}^{\dagger}\right) \operatorname{dvol}_{S}=\mathcal{N}_{k} \delta_{k l} \\
\left\langle\vec{\psi}_{L_{i}^{+}} \mid \vec{\psi}_{L_{j}^{+}}\right\rangle & =m_{*}^{2} \int_{S} \operatorname{Tr}\left(\vec{\psi}_{L_{i}^{+}} \cdot \vec{\psi}_{L_{j}^{+}}^{\dagger}\right) \operatorname{dvol}_{S}=\mathcal{N}_{i} \delta_{i j}
\end{aligned}
$$


we obtain upon dimensional reduction of (2.1) the $4 \mathrm{~d}$ superpotential

$$
W_{\text {tree }}^{4 \mathrm{~d}}=-i \sum_{\alpha, k} \mathcal{N}_{k} m_{k} H_{k}^{\alpha^{+}} H_{k}^{\alpha^{-}}+\text {Yukawas }
$$

with a very simple diagonal structure for the mass terms. Now, when adding the effect of $W_{\mathrm{np}}^{4 \mathrm{~d}}$, it is easy to see that such diagonal structure will be broken, and that in order to restore it we must redefine our fields. Indeed, the dimensional reduction of (3.8) gives

$$
W_{\mathrm{np}}^{4 \mathrm{~d}}=\epsilon \sum_{i j} \mu_{\mathrm{np}}^{i j} C_{i} C_{j}+\epsilon \sum_{i j k} h_{\mathrm{np}}^{i j k} C_{i} C_{j} C_{k}
$$

where now

$$
\begin{aligned}
& \mu_{\mathrm{np}}^{i j}=m_{*}^{2} d_{a b c} \int_{S} \operatorname{det}\left(\vec{\psi}_{a}^{\theta i},\left[\vec{F}_{y}^{\theta}\right]_{b},\left[D_{x} \vec{\psi}^{\theta j}\right]_{c}\right)-\operatorname{det}\left(\vec{\psi}_{a}^{\theta i},\left[\vec{F}_{x}^{\theta}\right]_{b},\left[D_{y} \vec{\psi}^{\theta j}\right]_{c}\right) \operatorname{dvol} \operatorname{lol}_{S} \\
& h_{\mathrm{np}}^{i j k}=m_{*} d_{a b c} \int_{S} \operatorname{det}\left(\vec{\psi}_{a}^{\theta i},\left[D_{y} \vec{\psi}^{\theta j}\right]_{b},\left[D_{x} \vec{\psi}^{\theta k}\right]_{c}\right)-\operatorname{det}\left(\vec{\psi}_{a}^{\theta i},\left[D_{x} \vec{\psi}^{\theta j}\right]_{b},\left[D_{y} \vec{\psi}^{\theta k}\right]_{c}\right) \operatorname{dvol}_{S}
\end{aligned}
$$

Here $d_{a b c}=\operatorname{STr}\left(\mathfrak{t}_{a}, \mathfrak{t}_{b}, \mathfrak{t}_{c}\right)$, and $D_{x, y}$ act on each component of the vector $\vec{\psi}^{\theta}$, defined as

$$
\vec{\psi}^{\theta}=\Theta \cdot \vec{\psi}=\left(\begin{array}{c}
\psi_{\bar{x}} \\
\psi_{\bar{y}} \\
\theta \chi_{x y}
\end{array}\right) \quad \Theta=\left(\begin{array}{ccc}
1 & 0 & 0 \\
0 & 1 & 0 \\
0 & 0 & \theta
\end{array}\right)
$$

Finally, we have also defined the background vectors

$$
\vec{F}_{x}^{\theta}=\left(\begin{array}{c}
\left\langle F_{x \bar{x}}\right\rangle \\
\left\langle F_{x \bar{y}}\right\rangle \\
\partial_{x}\left(\theta\left\langle\Phi_{x y}\right\rangle\right)
\end{array}\right) \quad \vec{F}_{y}^{\theta}=\left(\begin{array}{c}
\left\langle F_{y \bar{x}}\right\rangle \\
\left\langle F_{y \bar{y}}\right\rangle \\
\partial_{y}\left(\theta\left\langle\Phi_{x y}\right\rangle\right)
\end{array}\right)
$$

that, similarly to $\vec{\psi}$ and $\vec{\psi}^{\theta}$, can be decomposed as $\vec{F}_{m}^{\theta}=\left[\vec{F}_{m}^{\theta}\right]_{\alpha} \mathfrak{t}_{\alpha}$, with $\mathfrak{t}_{\alpha} \in \mathfrak{g}_{p}$. This time, however, the generators $\mathfrak{t}_{\alpha}$ will belong to the Cartan subalgebra of $\mathfrak{g}_{p}$, as a consequence of the intersecting 7-brane setup of section 2 .

It is useful to rewrite the mass term (3.18a) in the form

$$
\mu_{\mathrm{np}}^{i j}=m_{*}^{2} \int_{S} \operatorname{Tr}\left(\vec{\psi}^{\theta i} \cdot \tilde{\mathbf{K}} \cdot \vec{\psi}^{\theta j}\right) \operatorname{dvol}_{S}
$$

where the operator $\tilde{\mathbf{K}}$ is given by

$$
\tilde{\mathbf{K}}=\left(\begin{array}{ccc}
0 & -\mathcal{K}_{\bar{z}} & \mathcal{K}_{\bar{y}} \\
\mathcal{K}_{\bar{z}} & 0 & -\mathcal{K}_{\bar{x}} \\
-\mathcal{K}_{\bar{y}} & \mathcal{K}_{\bar{x}} & 0
\end{array}\right) \quad \mathcal{K}_{\bar{m}}=\left\{F_{y \bar{m}}^{\theta}, D_{x} \cdot\right\}-\left\{F_{x \bar{m}}^{\theta}, D_{y} \cdot\right\}
$$

and where $F_{x \bar{m}}^{\theta}=\left(\vec{F}_{x}^{\theta}\right)_{\bar{m}}, F_{y \bar{m}}^{\theta}=\left(\vec{F}_{y}^{\theta}\right)_{\bar{m}}$. That is

$$
F_{l \bar{n}}^{\theta}=\left\langle F_{l \bar{n}}\right\rangle, \quad F_{l \bar{z}}^{\theta}=\partial_{x}\left(\theta\left\langle\Phi_{x y}\right\rangle\right) \quad l, n=x, y
$$


Comparing (3.21) to the tree-level 2-point function (3.11a), we have performed the replacement $\bar{\partial}_{\mathbf{A}} \rightarrow \Theta \tilde{\mathbf{K}} \Theta$. This new operator does not need to be diagonal on the eigenvectors of the Laplace operator $\bar{\partial}_{\mathbf{A}}^{\dagger} \bar{\partial}_{\mathbf{A}}$, as it was the case for $\bar{\partial}_{\mathbf{A}}$. Recall from section 2 and appendix A that the operators $D_{x, y}$ act as creation operators on the zero mode wavefunctions $\vec{\psi}_{\alpha^{+}}^{L}$ for the chiral fields $L^{\alpha^{+}}$, and so $D_{x, y} \vec{\psi}_{\alpha^{+}}^{L}$ corresponds to the wavefunction of a massive mode. In particular, if in (3.21) we substitute $\vec{\psi}^{j} \rightarrow \vec{\psi}^{L}$ the integral will in general not vanish, producing non-vanishing couplings $\mu_{\mathrm{np}}^{j k} H_{k}^{\alpha^{-}} L_{j}^{\alpha^{+}}$for some $H_{k}^{\alpha^{-}}$. This will result in a $4 \mathrm{~d}$ effective superpotential of the form

$$
i W_{\text {total }}^{4 \mathrm{~d}}=\frac{1}{2} \sum_{\alpha, k}\left(\begin{array}{lll}
L_{j}^{\alpha^{+}} & H_{k}^{\alpha^{+}} & H_{k}^{\alpha^{-}}
\end{array}\right)\left(\begin{array}{ccc}
0 & 0 & i \epsilon \mu_{\mathrm{np}}^{j k} \\
0 & 0 & \mathcal{N}_{k} m_{k} \\
i \epsilon \mu_{\mathrm{np}}^{j k} & \mathcal{N}_{k} m_{k} & 0
\end{array}\right)\left(\begin{array}{c}
L_{j}^{\alpha^{+}} \\
H_{k}^{\alpha^{+}} \\
H_{k}^{\alpha^{-}}
\end{array}\right)+\ldots
$$

and so, in order to recover the diagonal structure of the tree-level superpotential (3.16) we must redefine our fields. In particular, we obtain that the new zero mode is given by

$$
\hat{L}_{j}^{\alpha^{+}}=L_{j}^{\alpha^{+}}-i \epsilon \sum_{k} \mu_{\mathrm{np}}^{j k}\left(\mathcal{N}_{k} m_{k}\right)^{-1} H_{k}^{\alpha^{+}}
$$

In addition, we will have to redefine our massive modes as $H_{k} \rightarrow \hat{H}_{k}$. This new set $\left\{\hat{H}_{k}\right\}$ of massive modes can be safely discarded from the superpotential at energies below $m_{k}$ [44], obtaining an effective superpotential that only depends on the new zero modes $\hat{L}_{j}$

$$
W_{\text {eff }}^{4 \mathrm{~d}}=\sum_{i j k}\left(\hat{h}^{i j k}+\epsilon \hat{h}_{\mathrm{np}}^{i j k}\right) \hat{L}_{i} \hat{L}_{j} \hat{L}_{k}
$$

Note however that the holomorphic Yukawa couplings $\hat{h}^{i j k}$ and $\hat{h}_{\mathrm{np}}^{i j k}$ are no longer the ones defined in (3.11b) and (3.18b), since now we are expressing everything in the hatted basis of new zero modes (3.25). The new couplings $\hat{h}^{i j k}$ will then be a linear combination of the previous ones $h^{i j k}$, in a way consistent with (3.25). Schematically, we will have

$$
\hat{h}^{L L L}=h^{L L L}-i \epsilon \sum_{H} \frac{\mu_{\mathrm{np}}^{L H}}{m_{H}} h^{L L H}+\mathcal{O}\left(\epsilon^{2}\right)
$$

where $h^{L L L}$ is an unhatted Yukawa coupling involving three tree-level zero modes $L$, and $h^{L L H}$ are Yukawa coupling involving two zero modes and one massive mode. Similar statements apply to $\hat{h}_{\mathrm{np}}^{i j k}$ and so we obtain the Yukawa structure

$$
\hat{h}^{L L L}=h^{L L L}+\epsilon\left(h_{\mathrm{np}}^{L L L}-i \sum_{H} \frac{\mu_{\mathrm{np}}^{L H}}{m_{H}} h^{L L H}\right)+\mathcal{O}\left(\epsilon^{2}\right)
$$

which should be compared with the expression (3.1) advanced at the beginning of this section. Clearly, we have that $Y_{\text {tree }}=h^{L L L}$ and that $Y_{\text {np }}$ is suppressed with respect to $Y_{\text {tree }}$ by the small parameter $\epsilon$. The main contribution to $Y_{\mathrm{np}}$ is given by the quantity in brackets and, although not clear at this point, there can be non-trivial cancellations between the two factors therein. 
In fact, the above sketchy expressions can be made more precise by the following observation. It is easy to convince oneself that the couplings $\hat{h}^{i j k}$ and $\hat{h}_{\mathrm{np}}^{i j k}$ may be easily computed from the rhs of (3.11b), respectively (3.18b), by simply replacing the wavefunctions $\vec{\psi}^{j}$ there by the linear combination of wavefunctions

$$
\vec{\psi}^{\hat{L}_{j}}=\vec{\psi}^{L_{j}}-i \epsilon \sum_{k} \frac{\mu_{\mathrm{np}}^{j k}}{\mathcal{N}_{k} m_{k}} \vec{\psi}^{H_{k}^{+}}
$$

that correspond to the corrected zero modes (3.25). It is interesting to note that this new zero modes no longer satisfy the classical zero mode equation $\bar{\partial}_{\mathbf{A}} \vec{\psi}=0$, but rather

$$
\bar{\partial}_{\mathbf{A}} \vec{\psi}^{\hat{L}_{j}}=\epsilon \sum_{k} \mu_{\mathrm{np}}^{j k} \mathcal{N}_{k}^{-1}\left(\vec{\psi}^{H_{k}^{-}}\right)^{\dagger}
$$

On the other hand, from (3.21) and the fact that $\vec{\psi}_{\alpha^{-}}^{H_{i}^{-}}$is a complete basis of wavefunctions for the sector $\alpha^{-}$one can deduce that

$$
\sum_{k} \mu_{\mathrm{np}}^{j k} \mathcal{N}_{k}^{-1}\left(\vec{\psi}^{H_{k}^{-}}\right)^{\dagger}=\Theta \tilde{\mathbf{K}} \Theta \vec{\psi}^{L_{j}}
$$

and so

$$
\bar{\partial}_{\mathbf{A}} \vec{\psi}^{\hat{L}_{j}}=\epsilon \Theta \tilde{\mathbf{K}} \Theta \vec{\psi}^{\hat{L}_{j}}+\mathcal{O}\left(\epsilon^{2}\right)
$$

This last equation will be particularly relevant when comparing the present $4 \mathrm{~d}$ approach to the $8 \mathrm{~d}$ approach discussed in the next subsection.

Finally, note that in deriving (3.18) we have used the classical values of $\langle A\rangle$ and $\langle\Phi\rangle$. One can however show that such values are also shifted by the effect of $W_{\mathrm{np}}^{4 \mathrm{~d}}$. This can be seen from the fact that the massive 7-brane bulk fields $X_{l}$ have a term $m_{X}\left(X_{l}-\left\langle X_{l}\right\rangle\right)^{2}$ generated at tree-level and a linear term $\epsilon \lambda^{l} X_{l}$ contained in $W_{\mathrm{np}}^{4 \mathrm{~d}}$, with

$$
\lambda^{l}=m_{*}^{3} d_{a b c} \int_{S} \operatorname{det}\left(\vec{\psi}_{\theta a}^{X_{l}},\left[\vec{F}_{y}^{\theta}\right]_{b},\left[\vec{F}_{x}^{\theta}\right]_{c}\right)-\operatorname{det}\left(\vec{\psi}_{\theta a}^{X_{l}},\left[\vec{F}_{x}^{\theta}\right]_{b},\left[\vec{F}_{y}^{\theta}\right]_{c}\right) \operatorname{dvol}_{S}
$$

Hence, unless $m_{X}\left\langle X_{l}\right\rangle \gg \lambda^{l}$ the vev of $X_{l}$ will be shifted by the non-perturbative effect by a non-negligible amount compared to $\epsilon$, and so the same will apply to $\langle A\rangle$ and $\langle\Phi\rangle$. This would not only affect quantities like $\mu_{\mathrm{np}}$ and $h_{\mathrm{np}}^{i j k}$, but also the tree-level mass terms (3.11a) via a shift in the operator (3.12). In fact, the latter effect will arise at first order in $\epsilon$ and so it will correct (3.28) non-trivially. While one may compute the effect of (3.33) within the present $4 \mathrm{~d}$ approach, let us turn our attention to a $8 \mathrm{~d}$ description of the same physics. As we will see, the latter will provide a systematic approach to compute this and all the non-perturbative effects that we have discussed.

\section{$3.28 \mathrm{~d}$ approach}

An interesting point regarding the $4 \mathrm{~d}$ analysis above is that the two superpotentials $W_{\text {tree }}^{4 \mathrm{~d}}$ and $W_{\mathrm{np}}^{4 \mathrm{~d}}$ have very different origin. On the one hand, $W_{\text {tree }}^{4 \mathrm{~d}}$ is obtained from the dimensional reduction of the $8 \mathrm{~d}$ field theory on the worldvolume of a stack of 7-branes, and in 
particular from reducing the functional (2.1) that depends on the $8 \mathrm{~d}$ fields $\left(A_{\bar{m}}, \Phi_{x y}\right)$. On the other hand, $W_{\mathrm{np}}^{4 \mathrm{~d}}$ arises at the level of the $4 \mathrm{~d}$ effective action, via the expression (3.2). This means that, just like $f_{7 \mathrm{np}}, W_{\mathrm{np}}^{4 \mathrm{~d}}$ should be defined as a function of the $4 \mathrm{~d}$ fields $\left\{C_{i}\right\}=\left\{L_{j}, H_{k}, X_{l}\right\}$, rather than $\left(A_{\bar{m}}, \Phi_{x y}\right)$.

Nevertheless, as it is clear from eq. (3.9), both superpotentials may be put on equal footing, in the sense that $W_{\text {total }}^{4 \mathrm{~d}}=W_{\text {tree }}^{4 \mathrm{~d}}+W_{\mathrm{np}}^{4 \mathrm{~d}}$ may be expressed as a sum of two functionals that depend on the $8 \mathrm{~d}$ fields $\left(A_{\bar{m}}, \Phi_{x y}\right)$. The reason for this is that, when carrying the $4 \mathrm{~d}$ effective theory analysis, it was necessary to consider the full spectrum of 7 -brane massive modes $\left\{C_{i}\right\}$, which contain the same information as the $8 \mathrm{~d}$ fields $\left(A_{\bar{m}}, \Phi_{x y}\right)$. As a consequence, rather than expressing (3.9) in terms of the classical $4 \mathrm{~d}$ fields $\left\{C_{i}\right\}$, one may analyze $W_{\text {total }}^{4 \mathrm{~d}}$ directly in terms of $\left(A_{\bar{m}}, \Phi_{x y}\right)$ and obtain the same results. Notice that if we variate $W_{\text {total }}^{4 \mathrm{~d}}$ with respect to $\left(A_{\bar{m}}, \Phi_{x y}\right)$ we will obtain a set of BPS equations that are different from (2.3), and that this implies a new set of background values and zero mode wavefunctions for the 7 -brane fields $(A, \Phi)$, slightly different to those obtained in section 2 . This is indeed what we expect from the results of the $4 \mathrm{~d}$ approach. The fact that we have new values for $\langle A\rangle$ and $\langle\Phi\rangle$ corresponds to a shift in the 7-brane vacuum induced by the linear terms $\epsilon \lambda^{l} X_{l}$ in the $4 \mathrm{~d}$ effective theory, and the fact that we have a new set of zero mode wavefunctions corresponds to the result that in the $4 \mathrm{~d}$ theory the true zero modes are given by (3.25).

In the following we will analyze $W_{\text {total }}^{4 \mathrm{~d}}$ from this $8 \mathrm{~d}$ point of view, in which the main objects are given by the $8 \mathrm{~d}$ fields $\left(A_{\bar{m}}, \Phi_{x y}\right)$. Since now we have a description of the perturbative + non-perturbative dynamics in terms of the $8 \mathrm{~d}$ functional (3.9), one may wonder if there could be an underlying $8 \mathrm{~d}$ field theory from which $W_{\text {total }}^{4 \mathrm{~d}}$ could be derived, as it is the case for $W_{\text {tree }}^{4 \mathrm{~d}}$. While this latter point is more speculative, it fits nicely with the set of ideas put forward in [25], which analyzed the contribution of non-perturbative effects to $4 \mathrm{~d}$ effective superpotentials from a higher dimensional viewpoint. Indeed, in the scheme of [25] (see also [24, 45]) one would trade the 4d non-perturbative field theory effect by a deformation of the 10d classical supergravity background that engineers the same $4 \mathrm{~d}$ physics, and then extract the non-perturbative physics from a 10d supergravity analysis of this new background.

For instance, in type IIB/F-theory compactifications on warped Calabi-Yau manifolds a gaugino condensing D7-brane would be 'backreacted' to a so-called $\beta$-deformation of the background which, among other things, involves the introduction of 3-form fluxes of the IASD kind, as in [46]. While it is not known how to construct compact examples of these $\beta$-deformed backgrounds, it is possible to implement $\beta$-deformations on local Calabi-Yau geometries [24, 45-47]. In particular, one may do so around a local type IIB/F-theory model of intersecting 7-branes. Then, in this deformed 10d background 7-branes should deform their 8d action, and in particular develop a new superpotential that would take into account all the non-perturbative effects computed in the standard $4 \mathrm{~d}$ approach.

As pointed out in [23] this is indeed the case, with the new superpotential looking precisely like (3.9). The derivation can be more clearly done in the type IIB orientifold limit of F-theory and it goes as follows. In a $\beta$-deformed background a D7-brane wrapping 
a 4-cycle $S$ develops a superpotential of the form [48]

$$
W_{\text {total }}=W_{\mathrm{CY}}+W_{\beta}=\frac{\mu_{5}}{2 \pi} \int_{S} \operatorname{Tr}\left(\chi_{2} \wedge F\right)+\epsilon \frac{\mu_{3}}{8 \pi^{2}} \int_{S} \mathrm{~S} \operatorname{Tr}\left(\chi_{0} F \wedge F\right)
$$

with $\mu_{p}=(2 \pi)^{-p} \alpha^{\prime-\frac{p+1}{2}}$ and $\chi_{2}$ a holomorphic $(2,0)$-form locally defined around $S$ and such that

$$
\mathrm{d} \chi_{2}=\Omega
$$

Just like $\log h$ in (3.5), $\chi_{2}$ needs to be Taylor expanded and pulled-back into $S$

$$
\chi_{2}=\left.\chi_{2}\right|_{S}+m_{\Phi}^{-2} \Phi^{z}\left[\mathcal{L}_{z} \chi_{2}\right]_{S}+m_{\Phi}^{-4}\left(\Phi^{z}\right)^{2}\left[\mathcal{L}_{z}^{2} \chi_{2}\right]_{S}+\ldots
$$

where now $m_{\Phi}^{-2}=2 \pi \alpha^{\prime}$. Since in our local coordinate system $\mathcal{L}_{z} \chi_{2}=\iota_{z} \Omega=d x \wedge d y$ and $\mathcal{L}_{z}^{2} \chi_{2}=0$, we have that

$$
W_{\mathrm{CY}}=\frac{\mu_{5}}{2 \pi} \int_{S} \operatorname{Tr}\left(\chi_{2} \wedge F\right)=\left.\frac{\mu_{5}}{2 \pi} \int_{S} \chi_{2}\right|_{S} \wedge \operatorname{Tr} F+\frac{\mu_{3}}{4 \pi^{2}} \int_{S} \operatorname{Tr}\left(\Phi^{z} F\right) \wedge d x \wedge d y
$$

This reproduces $(2.1)$ by choosing $\chi_{2}$ such that $\left.\chi_{2}\right|_{S}=0$, and substituting $\Phi^{z} \rightarrow \Phi_{x y}$ and $\mu_{3} / 4 \pi^{2} \rightarrow m_{*}^{4}$. Note that from the last identification it follows the second equation in (2.13), which we also expect to be valid in a general F-theory compactification.

On the other hand, the extra piece $W_{\beta}$ is specified by a holomorphic 0 -form $\chi_{0}$, which is proportional to $\log h$, with $h$ some divisor function. Again, $\left.\chi_{0}\right|_{S}$ is a constant and so

$$
\begin{aligned}
\epsilon^{-1} W_{\beta} & =\frac{\mu_{3}}{8 \pi^{2}} \int_{S} \operatorname{STr}\left(\chi_{0} F \wedge F\right)=\frac{\mu_{3}}{8 \pi^{2}} \int_{S} \chi_{0} \operatorname{Tr}(F \wedge F)+\frac{\mu_{3}}{8 \pi^{2}} \int_{S} \frac{\partial_{z} \chi_{0}}{m_{\Phi}^{2}} \operatorname{Tr}\left(\Phi^{z} F \wedge F\right)+\ldots \\
& =\left.\chi_{0}\right|_{S} \mu_{3} N_{D 3}+\frac{\mu_{3}}{8 \pi^{2}} \int_{S} \theta \operatorname{Tr}\left(\Phi^{z} F \wedge F\right)+\ldots
\end{aligned}
$$

where we have defined $\left.\theta \equiv m_{\Phi}^{-2} \partial_{z} \chi_{0}\right|_{S}$ and ignored higher powers of $\Phi^{z}=\Phi_{x y}$. Hence, up to constant terms and up to linear order in $\Phi_{x y}$ we are left with the superpotential

$$
W_{\text {total }}=m_{*}^{4}\left[\int_{S} \operatorname{Tr}\left(\Phi_{x y} F\right) \wedge d x \wedge d y+\frac{\epsilon}{2} \int_{S} \theta \operatorname{Tr}\left(\Phi_{x y} F \wedge F\right)\right]
$$

which indeed reproduces (3.9).

Let us now analyze this functional. Recall that in the $8 \mathrm{~d}$ approach the prescription is to variate the whole of (3.39) with respect to $\left(A_{\bar{m}}, \Phi_{x y}\right)$. We then obtain the F-term equations

$$
\begin{array}{r}
D_{\bar{m}} \Phi_{x y}+\epsilon\left[\left\{F_{\bar{m} x}, D_{y}\left(\theta \Phi_{x y}\right)\right\}+\left\{F_{y \bar{m}}, D_{x}\left(\theta \Phi_{x y}\right)\right\}+\left\{F_{x y}, D_{\bar{m}}\left(\theta \Phi_{x y}\right)\right\}\right]=0 \\
F \wedge d x \wedge d y+\epsilon \theta \frac{1}{2} F \wedge F=0
\end{array}
$$

with $\bar{m}=\bar{x}, \bar{y}$ and where we have used the Bianchi identity $D_{[m} F_{n p]}=0$. Clearly, eqs. (3.40) reduce to (2.3) for $\epsilon=0$, and become much harder to solve for non-vanishing $\epsilon$. This problem becomes somewhat easier for small values of $\epsilon$ (as is the case in our setup), since 
then we can apply perturbation theory to solve the new F-term equations. Indeed, one can then perform a perturbative expansion of the fields in the parameter $\epsilon$

$$
\Phi_{x y}=\Phi_{x y}^{(0)}+\epsilon \Phi_{x y}^{(1)}+\ldots \quad A_{\bar{m}}=A_{\bar{m}}^{(0)}+\epsilon A_{\bar{m}}^{(1)}+\ldots
$$

and then solve (3.40) order by order in $\epsilon$. It is then easy to see that to zeroth order these equations are given by the unperturbed F-term equations (2.3)

$$
\left(D_{\bar{m}} \Phi_{x y}\right)^{(0)}=F_{\bar{x} \bar{y}}^{(0)}=0
$$

while to first order we have

$$
\begin{aligned}
\left(D_{\bar{m}} \Phi_{x y}\right)^{(1)} & =-\left(\left\{F_{\bar{m} x}, D_{y}\left(\theta \Phi_{x y}\right)\right\}+\left\{F_{y \bar{m}}, D_{x}\left(\theta \Phi_{x y}\right)\right\}\right)^{(0)} \\
F^{(1)} \wedge d x \wedge d y & =-\theta \frac{1}{2} F^{(0)} \wedge F^{(0)}
\end{aligned}
$$

One should then solve these equations at the level of the 7-brane background and then at the level of the fluctuations. At the level of the background and at zeroth order in $\epsilon$ one may use, as in the previous section, the holomorphic gauge $\left\langle A_{\bar{x}}\right\rangle^{(0)}=\left\langle A_{\bar{y}}\right\rangle^{(0)}=0$, which drastically simplifies eqs. (3.43). Indeed, (3.43a) then reads

$$
\partial_{\bar{m}}\left\langle\Phi_{x y}\right\rangle^{(1)}=\partial_{\bar{m}}\left\langle A_{y} \partial_{x}\left(\theta \Phi_{x y}\right)-A_{x} \partial_{y}\left(\theta \Phi_{x y}\right)\right\rangle^{(0)}
$$

where similarly to the previous section we are assuming that $\left[\left\langle A_{m}\right\rangle^{(0)},\left\langle\Phi_{x y}\right\rangle^{(0)}\right]=0$. It is then easy to see that a solution to the $\mathcal{O}(\epsilon)$ background F-term equations is given by

$$
\begin{aligned}
\left\langle\Phi_{x y}\right\rangle^{(1)} & =\left\langle A_{y}\right\rangle^{(0)} \partial_{x}\left(\theta\left\langle\Phi_{x y}\right\rangle^{(0)}\right)-\left\langle A_{x}\right\rangle^{(0)} \partial_{y}\left(\theta\left\langle\Phi_{x y}\right\rangle^{(0)}\right) \\
\left\langle A_{\bar{x}}\right\rangle^{(1)} & =\frac{1}{2} \theta\left\langle A_{y} F_{x \bar{x}}-A_{x} F_{y \bar{x}}\right\rangle^{(0)} \\
\left\langle A_{\bar{y}}\right\rangle^{(1)} & =\frac{1}{2} \theta\left\langle A_{y} F_{x \bar{y}}-A_{x} F_{y \bar{y}}\right\rangle^{(0)}
\end{aligned}
$$

These corrections to the 7-brane background fields $\left\langle A_{\bar{m}}\right\rangle,\left\langle\Phi_{x y}\right\rangle$ correspond to the shifts induced by the operators (3.33) derived in the $4 \mathrm{~d}$ approach, as one may explicitly check. Notice that (3.45) is no longer compatible with the holomorphic gauge and that $\langle\Phi\rangle^{(1)}$ is not holomorphic. We then find that the correction $W_{\text {np }}$ to the 7-brane superpotential (2.1) spoils the holomorphic structure of the 7-brane background F-term in two different ways. On the one hand $\langle\Phi\rangle$ cannot be holomorphic and on the other hand we cannot maintain the holomorphic gauge $\left\langle A_{\bar{x}}\right\rangle=\left\langle A_{\bar{y}}\right\rangle=0$. These two non-holomorphic features arise already at first order in $\epsilon$ and, as we will see in section 4.2, they can be understood in terms of the holomorphic structure of a related non-commutative theory.

Similarly, one may solve for the zero mode wavefunctions order by order in $\epsilon$. First, the expansion (3.41) implies that at the level of the fluctuations we have a similar expansion

$$
\varphi_{x y}=\varphi_{x y}^{(0)}+\epsilon \varphi_{x y}^{(1)}+\ldots \quad a_{\bar{m}}=a_{\bar{m}}^{(0)}+\epsilon a_{\bar{m}}^{(1)}+\ldots
$$

Second, the equations of motion for the zeroth order wavefunctions $\left(\varphi_{x y}^{(0)}, a_{\bar{x}}^{(0)}, a_{\bar{y}}^{(0)}\right)$ are given by expanding (3.42) to first order in fluctuations. These are nothing but eqs. (2.7a) 
and $(2.7 \mathrm{~b})$ which, together with the D-term equation (2.7c), precisely specify the zero mode equations discussed in section 2.2. Thus, as expected, to zeroth order in $\epsilon$ our wavefunctions match the zero modes of the unperturbed 7-brane superpotential (2.1). Finally, the equations for $\left(\varphi_{x y}^{(1)}, a_{\bar{x}}^{(1)}, a_{\bar{y}}^{(1)}\right)$ are obtained by expanding (3.43) to first order in fluctuations. For instance, from (3.43a) one obtains

$$
\begin{aligned}
\partial_{\bar{m}} \varphi^{(1)}-i\left[a_{\bar{m}}^{(1)},\left\langle\Phi_{x y}\right\rangle^{(0)}\right]= & \left(\left\{F_{x \bar{m}}, D_{y}\left(\theta \Phi_{x y}\right)\right\}-\left\{F_{y \bar{m}}, D_{x}\left(\theta \Phi_{x y}\right)\right\}\right)^{(0)} \\
& +i\left[\left\langle A_{\bar{m}}\right\rangle^{(1)}, \varphi^{(0)}\right]+i\left[a_{\bar{m}}^{(0)},\left\langle\Phi_{x y}\right\rangle^{(1)}\right]
\end{aligned}
$$

where the first line of the rhs of (3.47) is still to be expanded to linear order in fluctuations. That is, we must replace

$$
\begin{aligned}
\left\{F_{x \bar{m}}, D_{y}\left(\theta \Phi_{x y}\right)\right\}^{(0)} \rightarrow & \left\{\left\langle F_{x \bar{m}}\right\rangle^{(0)}, \partial_{y}\left(\theta \varphi^{(0)}\right)-i \theta\left[\left\langle A_{y}\right\rangle^{(0)}, \varphi^{(0)}\right]\right\} \\
& +\left\{\partial_{x} a_{\bar{m}}^{(0)}-i\left[\left\langle A_{x}\right\rangle^{(0)}, a_{\bar{m}}^{(0)}\right], \partial_{y}\left(\theta\langle\Phi\rangle^{(0)}\right)\right\}
\end{aligned}
$$

and similarly for $\left\{F_{y \bar{m}}, D_{x}\left(\theta \Phi_{x y}\right)\right\}^{(0)}$. Note that the first line of (3.47) matches exactly with two of the three equations in (3.32), the remaining eq. arising from expanding (3.43b). Hence, we see that the linear combination of wavefunctions (3.29) found in the $4 \mathrm{~d}$ approach correspond to the solutions to the $\beta$-deformed zero mode equations. ${ }^{9}$ From this point of view, one can identify the corrections $\left(\varphi_{x y}^{(1)}, a_{\bar{x}}^{(1)}, a_{\bar{y}}^{(1)}\right)$ with linear combinations of tree-level wavefunctions for 7-brane massive modes $\left\{H_{k}\right\}$, a fact that will be used in appendix B in order to find explicit solutions for (3.46).

Iterating the above procedure one can obtain the background and wavefunction corrections $\vec{\psi}^{(n)}=\left(\varphi_{x y}^{(n)}, a_{\bar{x}}^{(n)}, a_{\bar{y}}^{(n)}\right)$ to order $\epsilon^{n}$. One then computes the corrected Yukawa couplings by inserting back these data into the functional (3.39) and integrating over the 4-cycle $S$. It is then easy to see that the corrected Yukawa couplings look like

$$
Y_{a b c}^{i j k}=\left(Y_{\text {tree }}^{(0)}\right)_{a b c}^{i j k}+\epsilon\left(Y_{\text {tree }}^{(1)}+Y_{\mathrm{np}}^{(0)}\right)_{a b c}^{i j k}+\mathcal{O}\left(\epsilon^{2}\right)
$$

in agreement with the previous structure (3.28). Indeed, $Y_{\text {tree }}^{(0)}$ is given by the previous expression (2.36), with all the three vectors in the determinant being zeroth order wavefunctions $\vec{\psi}^{(0)}$. The $\mathcal{O}(\epsilon)$ contribution $Y_{\text {tree }}^{(1)}$ arises from a similar expression, but now with two zeroth order wavefunction and one first order correction $\vec{\psi}^{(1)}$. More precisely

$$
\begin{gathered}
\left(Y_{\text {tree }}^{(1)}\right)_{a b c}^{i j k}=m_{*} f_{a b c} \int_{S}\left[\operatorname{det}\left(\left(\vec{\psi}_{a}^{i}\right)^{(1)},\left(\vec{\psi}_{b}^{j}\right)^{(0)},\left(\vec{\psi}_{c}^{k}\right)^{(0)}\right)+\operatorname{det}\left(\left(\vec{\psi}_{a}^{i}\right)^{(0)},\left(\vec{\psi}_{b}^{j}\right)^{(1)},\left(\vec{\psi}_{c}^{k}\right)^{(0)}\right)\right. \\
\left.+\operatorname{det}\left(\left(\vec{\psi}_{a}^{i}\right)^{(0)},\left(\vec{\psi}_{b}^{j}\right)^{(0)},\left(\vec{\psi}_{c}^{k}\right)^{(1)}\right)\right] d \operatorname{vol}_{S}
\end{gathered}
$$

Finally, the $\mathcal{O}(\epsilon)$ contribution $Y_{\mathrm{np}}^{(0)}$ arises from inserting zeroth order wavefunctions into $W_{\text {np }}$ in (3.39). That is

$$
\begin{aligned}
\left(Y_{\mathrm{np}}^{(0)}\right)_{a b c}^{i j k}= & m_{*} d_{a b c} \int_{S} \theta\left(\varphi_{x y}^{(0)}\right)_{a}^{i} \partial_{\langle A\rangle}\left(a^{(0)}\right)_{b}^{j} \wedge \partial_{\langle A\rangle}\left(a^{(0)}\right)_{c}^{k} \\
& + \text { cyclic permutations in } a b c
\end{aligned}
$$

\footnotetext{
${ }^{9}$ Obviously, the same thing will happen when we include the effect of $\left\langle A_{\bar{m}}\right\rangle^{(1)}$ and $\left\langle\Phi_{x y}\right\rangle^{(1)}$.
} 
where $\partial_{\langle A\rangle}=\partial-i\langle A\rangle^{(0)} \wedge$. As in our $4 \mathrm{~d}$ results, from these expressions we reproduce a Yukawa structure of the form (3.1). In particular, from $Y_{\text {tree }}^{(0)}$ we obtain a rank-one Yukawa matrix with $\mathcal{O}(1)$ coefficients, and so this contribution will play the role of $Y_{\text {tree }}$ in (3.1). The extra contribution to Yukawa couplings arising from non-perturbative physics will be given by $\epsilon\left(Y_{\text {tree }}^{(1)}+Y_{\mathrm{np}}^{(0)}\right)+\mathcal{O}\left(\epsilon^{2}\right)$ and, while with suppressed $\mathcal{O}(\epsilon)$ coefficients, will generically raise the total Yukawa matrix to its maximal rank.

\subsection{Non-commutative approach}

A quite interesting variation of the $8 \mathrm{~d}$ approach consist of expressing the $\beta$-deformed 7brane superpotential (3.34) in terms of $8 \mathrm{~d}$ non-commutative fields $\hat{A}_{\bar{m}}$ and $\hat{\Phi}$. Indeed, as shown in [23] and in appendix C, by applying a generalized Seiberg-Witten map the superpotential $W_{\mathrm{CY}}+W_{\beta}$ is transformed to

$$
\hat{W}=m_{*}^{4} \int_{S} \operatorname{Tr}(\hat{\Phi} \circledast \hat{F})
$$

up to $\mathcal{O}\left(\epsilon^{2}\right)$ terms. This new superpotential is a non-commutative version of $W_{\mathrm{CY}}$ in the sense that it has the same structure, but now the 7-brane fields $\hat{A}$ and $\hat{\Phi}$ should be multiplied according to a non-commutative version of the usual scalar and wedge products. More precisely, two scalar functions $f$ and $g$ will be multiplied by the holomorphic Moyal product, which reads

$$
f * g=f g+\frac{i}{2} \epsilon \theta^{i j} \partial_{i} f \partial_{j} g+\mathcal{O}\left(\epsilon^{2}\right) \quad \theta^{y x}=-\theta^{x y}=\theta
$$

whenever $\theta$ is a constant. For non-constant $\theta=\theta(x, y)$ this definition has to be modified, as explained in appendix B of [16], where also the non-commutative version $\circledast$ of the ordinary wedge product was discussed for this case.

In fact, the non-commutative superpotential (3.52) was already proposed in [16] as a way to overcome the Yukawa rank one problem discussed in section 2. As a proof of concept, in [16] an explicit example was analyzed, for which the Yukawa couplings were computed using the non-commutative approach. This showed that, indeed, the Yukawa rank one result no longer holds when one considers the non-commutative deformation (3.52) of the superpotential (2.1).

While perhaps less intuitive, the non-commutative approach has a number of advantages for the computation of wavefunctions and Yukawa couplings. In particular, as we will discuss in section 5, it allows to realize that even if now the Yukawa matrices have maximal rank, still they do not depend on the worldvolume magnetic fluxes $\hat{F}$ on the 7-branes. As discussed in the introduction, this could still pose a severe phenomenological handicap for local F-theory models.

Just like for its commutative counterpart (2.1) we may compute the F-term equations for (3.52). Following [16] they read

$$
\begin{aligned}
\bar{\partial}_{A \circledast \hat{\Phi}} & =\bar{\partial} \hat{\Phi}-i[\hat{A}, \hat{\Phi}]_{*}=0 \\
\hat{F}^{(0,2)} & =\bar{\partial} \hat{A}-i \hat{A} \circledast \hat{A}=0
\end{aligned}
$$


where $\hat{A}=\hat{A}_{\bar{x}} d \bar{x}+\hat{A}_{\bar{y}} d \bar{y}$ is a $(0,1)$-form. As in section 2 , these equations greatly simplify if we take a non-commutative version of the holomorphic gauge of [15], namely setting $\left\langle\hat{A}_{\bar{m}}\right\rangle=$ 0 for $\bar{m}=\bar{x}, \bar{y}$. Indeed, we then have that at the level of the background they amount to set $\left\langle\hat{\Phi}_{x y}\right\rangle$ holomorphic. In addition, defining the non-commutative fields fluctuations as

$$
\hat{\Phi}_{x y}=\left\langle\hat{\Phi}_{x y}\right\rangle+\hat{\varphi}_{x y} \quad \hat{A}_{\bar{m}}=\left\langle\hat{A}_{\bar{m}}\right\rangle+\hat{a}_{\bar{m}}
$$

and expanding (3.54) to first order in fluctuations we find the wavefunction equations

$$
\begin{aligned}
& \bar{\partial}_{\bar{m}} \hat{\varphi}_{x y}-i\left[\hat{a}_{\bar{m}},\left\langle\hat{\Phi}_{x y}\right\rangle\right]+\epsilon \sum_{i j}\left\{\partial_{i} \hat{a}_{\bar{m}}, \partial_{j}\left(\theta^{i j}\left\langle\hat{\Phi}_{x y}\right\rangle\right)\right\}=\mathcal{O}\left(\epsilon^{2}\right) \\
& \bar{\partial}_{\bar{x}} \hat{a}_{\bar{y}}-\bar{\partial}_{\bar{y}} \hat{a}_{\bar{x}}=\mathcal{O}\left(\epsilon^{2}\right)
\end{aligned}
$$

where now all products are commutative, $i, j=x, y$ and again $\theta^{y x}=-\theta^{x y}=\theta$.

Besides modifying the wavefunctions, the non-commutative superpotential (3.52) also induces $\theta$ depending corrections to the Yukawa couplings. In particular, $\hat{W}$ includes the trilinear term

$$
\hat{W}_{\text {Yuk }}=-i m_{*}^{4} \int_{S} \operatorname{Tr}(\hat{A} \circledast \hat{A} \circledast \hat{\Phi})
$$

which has the $\epsilon$ expansion

$$
\hat{W}_{\text {Yuk }}=\hat{W}_{0}+\epsilon \hat{W}_{1}+\mathcal{O}\left(\epsilon^{2}\right)
$$

To zeroth order in $\epsilon$ such trilinear term reads

$$
\hat{W}_{0}=-i m_{*}^{4} \int_{S} \operatorname{Tr}(\hat{A} \wedge \hat{A} \wedge \hat{\Phi})
$$

while the first order correction turns out to be

$$
\hat{W}_{1}=m_{*}^{4} d_{a b c} \int_{S} \theta \partial \hat{A}_{a} \wedge \partial \hat{A}_{b} \hat{\Phi}_{c x y}+\text { cyclic permutations in } a, b, c
$$

where a surface term has been dropped. The $\mathcal{O}(\epsilon)$ corrections to the Yukawa couplings due to $\hat{W}_{1}$ are the non-commutative counterpart of the non-perturbative couplings of equation (3.51). There will be in addition an $\mathcal{O}(\epsilon)$ correction analogous to (3.50), arising from inserting the the zero mode wavefunctions solving (3.56) within $\hat{W}_{0}$, and then expanding to first order in $\epsilon$.

We will continue the discussion of non-commutative zero modes and Yukawa couplings in section 5, to which the reader may safely jump if not interested in the relation between commutative and non-commutative formalisms to be discussed in the next section. In particular, in subsection 4.2 we will relate the two set of equations (3.47) and (3.56a) by a Seiberg-Witten map and show that, up to $\mathcal{O}\left(\epsilon^{2}\right)$ corrections, solving one of them is equivalent to solving the other.

\section{Corrected wavefunctions}

As discussed in the previous section, the effect of a non-perturbative superpotential for the chiral matter fields of a local F-theory model can be understood in terms of a deformation 
of their internal wavefunctions. Namely, the new wavefunctions read

$$
\Psi=\Psi_{0}+\sum_{\lambda} c_{\lambda} \Psi_{\lambda}
$$

where $\Psi_{0}$ is the wavefunction for the chiral field before the non-perturbative effect has been taken into account, $\left\{\Psi_{\lambda}\right\}$ is an appropriate basis of wavefunctions and $c_{\lambda}$ are complex coefficients that vanish when the strength $\epsilon$ of the non-perturbative effect does.

In this section we describe the corrected wavefunction $\Psi$ in terms of the $8 \mathrm{~d}$ approach of section 3.2. That is, the new wavefunction $\Psi$ will be the solution of a new set of zero mode equations, which arise from deforming the previous F-term equations (2.3) to the more complicated ones (3.40). While computing $\Psi$ exactly is quite involved, one may simplify the problem by expanding $\Psi$ in the small parameter $\epsilon$ and then applying perturbation theory. In the following we will describe this perturbative strategy for the deformed Fterms of section 3.2, and obtain the equations for the $\mathcal{O}(\epsilon)$ correction to $\Psi_{0}$ in this case. In fact, the same perturbative strategy may be applied to the non-commutative F-term equations of section 3.3, where the new zero mode equations are given by (3.56). We will do so and show that, up to $\mathcal{O}\left(\epsilon^{2}\right)$ corrections, the commutative and non-commutative corrected wavefunctions are related by a simple Seiberg-Witten map.

As both kind of wavefunctions are equivalent, in section 5 we will focus on solving for corrected wavefunctions within the non-commutative formalism, leaving the computation in the commutative formalism for appendix B. It will become clear from the latter analysis that a basis of wavefunctions $\left\{\Psi_{\lambda}\right\}$ suitable to solve (4.1) is given by the tower of unperturbed massive modes at the matter curve (i.e., those wavefunctions satisfying eq. (2.11)) as already suggested by the $4 \mathrm{~d}$ analysis of section 3.1 .

\subsection{Wavefunctions and perturbation theory}

Let us consider a chiral zero mode wavefunction $\Psi_{0}$ in the absence of non-perturbative effect $/ \beta$-deformation. By the results of section $2, \Psi_{0}$ must satisfy the equation

$$
\mathbf{D}_{\mathbf{A}} \Psi_{0}=0
$$

where the matrix operator $\mathbf{D}_{\mathbf{A}}$ and the wavefunction vector $\Psi$ are defined as in (2.10).

Let us now assume that the system is perturbed such that the zero mode equations are modified to

$$
\mathbf{D}_{\mathbf{A}} \Psi=\mathbf{K} \Psi
$$

where $\mathbf{K}$ is a linear operator that may contain derivatives and functions but does not depend on $\Psi$, and admits an expansion of the form

$$
\mathbf{K}=\epsilon \mathbf{K}^{(1)}+\epsilon^{2} \mathbf{K}^{(2)}+\ldots
$$

with $\epsilon$ a small parameter. It is then natural to consider an expansion of the form

$$
\Psi=\Psi^{(0)}+\epsilon \Psi^{(1)}+\epsilon^{2} \Psi^{(2)}+\ldots
$$


and to solve (4.3) order by order in $\epsilon$. Clearly, to zeroth order in $\epsilon$ we just need to impose $\Psi^{(0)}=\Psi_{0}$, while to $\mathcal{O}(\epsilon)$ we have

$$
\mathbf{D}_{\mathbf{A}} \Psi^{(1)}=\mathbf{K}^{(1)} \Psi_{0}
$$

Note that this perturbative method may be applied to solve any equation of motion of the form (4.3). ${ }^{10}$ In the following, however, we will focus on those corrections that only deform the F-term equations (2.7a) and (2.7b), and leave the D-term equation (2.7c) unchanged. This means that

$$
\mathbf{K}=\left(\begin{array}{ll}
0 & 0 \\
0 & \mathbf{k}
\end{array}\right) \quad \mathbf{k}=\epsilon \mathbf{k}^{(1)}+\epsilon^{2} \mathbf{k}^{(2)}+\ldots
$$

for some $3 \times 3$ submatrix k. Eq. (4.6) then reduces to the standard D-term equation and the deformed $\mathrm{F}$-term equations

$$
\bar{\partial}_{\mathbf{A}} \vec{\psi}^{(1)}=\mathbf{k}^{(1)} \vec{\psi}_{0}
$$

where $\vec{\psi}=\vec{\psi}_{0}+\epsilon \vec{\psi}^{(1)}+\ldots$ and $\bar{\partial}_{\mathbf{A}}$ are defined by (2.35) and (3.12), respectively.

\section{Commutative equations}

By the results of section 3.2, the wavefunction equations that arise from the $\beta$-deformed F-terms (3.40) are indeed of the form (4.3) and (4.7). More precisely we have that

$$
\mathbf{k}^{(1)}=-\Theta(\tilde{\mathbf{K}}+i \tilde{\mathbf{A}})^{(0)} \Theta
$$

with $\tilde{\mathbf{K}}$ and $\Theta$ defined as in (3.22) and (3.19), respectively. The operator $\tilde{\mathbf{A}}$ contains the terms arising from the second line of (3.47). Using the background solutions (3.45), we find that it reads

$$
\tilde{\mathbf{A}}=\left(\begin{array}{ccc}
0 & -\mathcal{A}_{\bar{z}} & \frac{1}{2} \mathcal{A}_{\bar{y}} \\
\mathcal{A}_{\bar{z}} & 0 & -\frac{1}{2} \mathcal{A}_{\bar{x}} \\
-\frac{1}{2} \mathcal{A}_{\bar{y}} & \frac{1}{2} \mathcal{A}_{\bar{x}} & 0
\end{array}\right) \quad \mathcal{A}_{\bar{m}}=\left[\left\langle F_{y \bar{m}}^{\theta} A_{x}-F_{x \bar{m}}^{\theta} A_{y}\right\rangle, \cdot\right]
$$

where $F_{n \bar{m}}^{\theta}$ is again given by (3.23). Finally, the superscript (0) in (4.9) indicates that within $\tilde{\mathbf{K}}$ and $\tilde{\mathbf{A}}$ one should take the uncorrected vevs $\left\langle A_{\bar{m}}\right\rangle^{(0)}$ and $\left\langle\Phi_{x y}\right\rangle^{(0)}$.

That is, we have that the $\mathcal{O}(\epsilon)$ wavefunction equation is given by (4.8) with

$$
\begin{aligned}
\mathbf{k}^{(1)}=- & -\Theta(\tilde{\mathbf{K}}+i \tilde{\mathbf{A}})^{(0)} \Theta \\
& -(\tilde{\mathbf{K}}+i \tilde{\mathbf{A}})=\left(\begin{array}{ccc}
0 & \mathcal{K}_{\bar{z}}+i \mathcal{A}_{\bar{z}} & -\left(\mathcal{K}_{\bar{y}}+\frac{i}{2} \mathcal{A}_{\bar{y}}\right) \\
-\left(\mathcal{K}_{\bar{z}}+i \mathcal{A}_{\bar{z}}\right) & 0 & \mathcal{K}_{\bar{x}}+\frac{i}{2} \mathcal{A}_{\bar{x}} \\
\mathcal{K}_{\bar{y}}+\frac{i}{2} \mathcal{A}_{\bar{y}} & -\left(\mathcal{K}_{\bar{x}}+\frac{i}{2} \mathcal{A}_{\bar{x}}\right) & 0
\end{array}\right)
\end{aligned}
$$

and

$$
\begin{aligned}
\mathcal{K}_{\bar{m}} & =\left\{F_{y \bar{m}}^{\theta}, D_{x} \cdot\right\}-\left\{F_{x \bar{m}}^{\theta}, D_{y} \cdot\right\} & \mathcal{A}_{\bar{m}} & =\left[\left\langle F_{y \bar{m}}^{\theta} A_{x}-F_{x \bar{m}}^{\theta} A_{y}\right\rangle, \cdot\right] \\
F_{l \bar{n}}^{\theta} & =\left\langle F_{l \bar{n}}\right\rangle \quad F_{l \bar{z}}^{\theta}=\partial_{l}\left(\theta\left\langle\Phi_{x y}\right\rangle\right) & l, n & =x, y
\end{aligned}
$$

\footnotetext{
${ }^{10}$ See for instance [41] for its application to compute chiral wavefunctions in warped backgrounds.
} 


\section{Non-commutative equations}

In order to write the analogous equations in the non-commutative formalism it is useful to consider the non-commutative version of the vectors (2.35) and (3.19)

$$
\hat{\psi}=\left(\begin{array}{c}
\hat{a}_{\bar{x}} \\
\hat{a}_{\bar{y}} \\
\hat{\varphi}_{x y}
\end{array}\right)=\hat{\psi}_{\alpha} \mathfrak{t}_{\alpha} \quad \hat{\psi}^{\theta}=\Theta \cdot \hat{\psi}=\left(\begin{array}{c}
\hat{a}_{\bar{x}} \\
\hat{a}_{\bar{y}} \\
\theta \hat{\varphi}_{x y}
\end{array}\right)
$$

So that eqs. (3.56) can be rewritten as

$$
\bar{\partial}_{\mathbf{A}} \hat{\psi}=\epsilon\left(\begin{array}{ccc}
0 & \mathcal{Q}_{\bar{z}}^{(0)} & 0 \\
-\mathcal{Q}_{\bar{z}}^{(0)} & 0 & 0 \\
0 & 0 & 0
\end{array}\right) \hat{\psi}+\mathcal{O}\left(\epsilon^{2}\right) \quad \mathcal{Q}_{\bar{m}}=\left\{F_{y \bar{m}}^{\theta}, \partial_{x} \cdot\right\}-\left\{F_{x \bar{m}}^{\theta}, \partial_{y} \cdot\right\}
$$

where as before $F_{n \bar{m}}^{\theta}$ is given by (3.23). This again implies a corrected zero mode wavefuction of the form

$$
\hat{\psi}=\hat{\psi}_{0}+\epsilon \hat{\psi}^{(1)}+\ldots \quad \text { with } \quad \bar{\partial}_{\mathbf{A}} \hat{\psi}_{0}=0, \quad \bar{\partial}_{\mathbf{A}} \hat{\psi}^{(1)}=\hat{\mathbf{k}}^{(1)} \hat{\psi}_{0}
$$

and

$$
\hat{\mathbf{k}}^{(1)}=\left(\begin{array}{ccc}
0 & \mathcal{Q}_{\bar{z}}^{(0)} & 0 \\
-\mathcal{Q}_{\bar{z}}^{(0)} & 0 & 0 \\
0 & 0 & 0
\end{array}\right)
$$

Finally, note that for $\theta \neq 0$ eq. (4.13) is equivalent to

$$
\bar{\partial}_{\mathbf{A}}^{\theta} \hat{\psi}^{\theta}=\epsilon \theta \hat{\mathbf{k}}^{(1)} \hat{\psi}^{\theta}+\mathcal{O}\left(\epsilon^{2}\right)
$$

with $\bar{\partial}_{\mathbf{A}}^{\theta}$ given by

$$
\bar{\partial}_{\mathbf{A}}^{\theta}=\left(\begin{array}{ccc}
0 & i\left[\theta\left\langle\Phi_{x y}\right\rangle^{(0)}, \cdot\right] & \partial_{\bar{y}} \\
-i\left[\theta\left\langle\Phi_{x y}\right\rangle^{(0)}, \cdot\right] & 0 & -\partial_{\bar{x}} \\
-\partial_{\bar{y}} & \partial_{\bar{x}} & 0
\end{array}\right)
$$

This alternative expression will be important in proving the equivalence between the commutative and non-commutative zero mode equations, to which we now turn.

\subsection{Relating commutative and non-commutative wavefunctions}

As pointed out in [23], the $8 \mathrm{~d} \beta$-deformed superpotential (3.34) can be expressed in terms of the non-commutative superpotential $\hat{W}$ in (3.52), upon applying a Seiberg-Witten map relating the non-commutative 7 -brane fields $(\hat{A}, \hat{\Phi})$ to the standard ones $(A, \Phi)$. As shown explicitly in appendix C.2 such SW map is given by

$$
\begin{aligned}
& \hat{A}_{\bar{m}}=A_{\bar{m}}+\tilde{A}_{\bar{m}}=A_{\bar{m}}-\frac{\epsilon}{2} \theta^{i j}\left\{A_{i}, \partial_{j} A_{\bar{m}}+F_{j \bar{m}}\right\}+\mathcal{O}\left(\epsilon^{2}\right) \\
& \hat{\Phi}_{x y}=\Phi_{x y}+\tilde{\Phi}_{x y}=\Phi_{x y}-\frac{\epsilon}{2}\left\{A_{i},\left(\partial_{j}+D_{j}\right)\left(\theta^{i j} \Phi_{x y}\right)\right\}+\mathcal{O}\left(\epsilon^{2}\right)
\end{aligned}
$$

where all the products in the rhs are standard ones. As in (3.56), $\theta^{y x}=-\theta^{x y}=\theta$. 
One can gain some intuition on the meaning of this Seiberg-Witten map by applying it at the level of the background. We have that $\left\langle\hat{A}_{\bar{m}}\right\rangle=\left\langle A_{\bar{m}}\right\rangle+\left\langle\tilde{A}_{\bar{m}}\right\rangle$, where

$$
\begin{aligned}
& \left\langle A_{\bar{m}}\right\rangle=\left\langle A_{\bar{m}}\right\rangle^{(0)}+\epsilon\left\langle A_{\bar{m}}\right\rangle^{(1)}+\mathcal{O}\left(\epsilon^{2}\right) \\
& \left\langle\tilde{A}_{\bar{m}}\right\rangle=\frac{\epsilon}{2} \theta\left\langle A_{x}\left(\partial_{y} A_{\bar{m}}+F_{y \bar{m}}\right)-A_{y}\left(\partial_{x} A_{\bar{m}}+F_{x \bar{m}}\right)\right\rangle^{(0)}+\mathcal{O}\left(\epsilon^{2}\right)
\end{aligned}
$$

and that $\left\langle\hat{\Phi}_{x y}\right\rangle=\left\langle\Phi_{x y}\right\rangle+\left\langle\tilde{\Phi}_{x y}\right\rangle$, with

$$
\begin{aligned}
& \left\langle\Phi_{x y}\right\rangle=\left\langle\Phi_{x y}\right\rangle^{(0)}+\epsilon\left\langle\Phi_{x y}\right\rangle^{(1)}+\mathcal{O}\left(\epsilon^{2}\right) \\
& \left\langle\tilde{\Phi}_{x y}\right\rangle=\epsilon\left\langle A_{x} \partial_{y}\left(\theta \Phi_{x y}\right)-A_{y} \partial_{x}\left(\theta \Phi_{x y}\right)\right\rangle^{(0)}+\mathcal{O}\left(\epsilon^{2}\right)
\end{aligned}
$$

Both in (4.19) and in (4.20) we have used our previous assumption that $\left\langle A_{m}\right\rangle^{(0)},\left\langle A_{\bar{m}}\right\rangle^{(0)}$ and $\left\langle\Phi_{x y}\right\rangle^{(0)}$ commute. In particular, taking the holomorphic gauge $\left\langle A_{\bar{m}}\right\rangle^{(0)}=0$ and the corresponding first order perturbations $\left\langle A_{\bar{m}}\right\rangle^{(1)}$ and $\left\langle\Phi_{x y}\right\rangle^{(1)}$ found in eqs. (3.45), we find that $\left\langle\tilde{A}_{\bar{m}}\right\rangle=-\epsilon\left\langle A_{\bar{m}}\right\rangle^{(1)}$ and $\left\langle\tilde{\Phi}_{x y}\right\rangle=-\epsilon\left\langle\Phi_{x y}\right\rangle^{(1)}$. Hence

$$
\begin{aligned}
\left\langle\hat{A}_{\bar{m}}\right\rangle & =0+\mathcal{O}\left(\epsilon^{2}\right) \\
\left\langle\hat{\Phi}_{x y}\right\rangle & =\left\langle\Phi_{x y}\right\rangle^{(0)}+\mathcal{O}\left(\epsilon^{2}\right)
\end{aligned}
$$

and so the background values for the non-commutative fields $\hat{A}, \hat{\Phi}$ correspond to those of the commutative fields $A, \Phi$ at zeroth order in $\epsilon$, that is before the $\beta$-deformation/nonperturbative effect was taken into account. Note in particular that in the non-commutative variables we recover the holomorphic structure (that is, the holomorphic gauge for $\langle\hat{A}\rangle$ and the fact that $\langle\hat{\Phi}\rangle$ is holomorphic) that was lost for $\langle A\rangle,\langle\Phi\rangle$ when we included the effect of the $\beta$-deformation. In this sense, the SW map (4.18) relates rather directly the holomorphic gauge introduced in [15] and its non-commutative version. We see that the non-commutative holomorphic gauge used in [16] is nothing but a deformation of the standard holomorphic gauge such that it makes the latter compatible with the $\beta$-deformed equations of motion.

In addition, the map (4.18) between commutative and non-commutative $8 \mathrm{~d}$ fields implies a well-defined dictionary between the corresponding 7-brane wavefunctions, and in particular between the $\theta$-corrected zero modes of appendix $\mathrm{B}$ and their non-commutative counterparts solving eqs. (3.56). Indeed, let us build explicitly such dictionary between commutative and non-commutative zero modes. For this we need to expand (4.18) to first order in the fluctuations $\left(\hat{a}_{\bar{m}}, \hat{\varphi}_{x y}\right)$ defined in $(3.55)$. We find

$$
\hat{a}_{\bar{m}}=a_{\bar{m}}+\tilde{a}_{\bar{m}} \quad \hat{\varphi}_{x y}=\varphi_{x y}+\tilde{\varphi}_{x y}
$$

where

$$
\begin{aligned}
\tilde{a}_{\bar{m}} & =\epsilon \theta\left[\left\{\left\langle A_{x}\right\rangle, \frac{1}{2}\left(\partial_{y}+D_{y}\right) a_{\bar{m}}\right\}-\left\{\left\langle A_{y}\right\rangle, \frac{1}{2}\left(\partial_{x}+D_{x}\right) a_{\bar{m}}\right\}\right] \\
& =\epsilon \theta\left(\left\{\left\langle A_{x}\right\rangle, \partial_{y} a_{\bar{m}}\right\}-\left\{\left\langle A_{y}\right\rangle, \partial_{x} a_{\bar{m}}\right\}\right) \\
\tilde{\varphi}_{x y} & =\epsilon\left[\left\{\left\langle A_{x}\right\rangle, \frac{1}{2}\left(\partial_{y}+D_{y}\right)\left(\theta \varphi_{x y}\right)\right\}-\left\{\left\langle A_{y}\right\rangle, \frac{1}{2}\left(\partial_{x}+D_{x}\right)\left(\theta \varphi_{x y}\right)\right\}\right] \\
& =\epsilon\left(\left\{\left\langle A_{x}\right\rangle, \partial_{y}\left(\theta \varphi_{x y}\right)\right\}-\left\{\left\langle A_{y}\right\rangle, \partial_{x}\left(\theta \varphi_{x y}\right)\right\}\right)
\end{aligned}
$$


up to $\mathcal{O}\left(\epsilon^{2}\right)$ corrections. This map can be expressed in a more compact way in terms of the vector $\hat{\psi}^{\theta}$ defined in (4.12). Indeed, we have that

$$
\hat{\psi}^{\theta}=\left(\mathbb{I}-\epsilon \theta \mathcal{Q}^{(0)}\right) \vec{\psi}^{\theta} \quad \mathcal{Q}=\left\{\left\langle A_{y}\right\rangle, \partial_{x} \cdot\right\}-\left\{\left\langle A_{x}\right\rangle, \partial_{y} \cdot\right\}
$$

with $\vec{\psi}^{\theta}$ given by (3.19).

Let us now see that (4.24) maps non-commutative zero modes to $\beta$-deformed zero modes. Combining the non-commutative zero mode equation (4.16) with (4.24) we obtain

$$
\bar{\partial}_{\mathbf{A}}^{\theta} \vec{\psi}^{\theta}=\epsilon \theta\left[\hat{\mathbf{k}}^{(1)}+\bar{\partial}_{\mathbf{A}}^{\theta} \mathcal{Q}^{(0)}\right] \vec{\psi}^{\theta}+\mathcal{O}\left(\epsilon^{2}\right)
$$

We may now perform an expansion on the wavefunction $\vec{\psi}^{\theta}$

$$
\vec{\psi}^{\theta}=\left(\vec{\psi}^{\theta}\right)^{(0)}+\epsilon\left(\vec{\psi}^{\theta}\right)^{(1)}+\ldots
$$

and write down (4.25) order by order in $\epsilon$. At zeroth order we have that

$$
\bar{\partial}_{\mathbf{A}}^{\theta}\left(\vec{\psi}^{\theta}\right)^{(0)}=0 \quad \Longleftrightarrow \quad \bar{\partial}_{\mathbf{A}} \vec{\psi}^{(0)}=0
$$

and to first order we obtain

$$
\begin{aligned}
\bar{\partial}_{\mathbf{A}}^{\theta}\left(\vec{\psi}^{\theta}\right)^{(1)} & =\theta\left[\hat{\mathbf{k}}^{(1)}+\left[\bar{\partial}_{\mathbf{A}}^{\theta}, \mathcal{Q}^{(0)}\right]\right]\left(\vec{\psi}^{\theta}\right)^{(0)} \\
& =\theta\left(\begin{array}{ccc}
0 & \mathcal{Q}_{\bar{z}}+i \mathcal{R}_{\bar{z}}-\mathcal{Q}_{\bar{y}} \\
-\left(\mathcal{Q}_{\bar{z}}+i \mathcal{R}_{\bar{z}}\right) & 0 & \mathcal{Q}_{\bar{x}} \\
\mathcal{Q}_{\bar{y}} & -\mathcal{Q}_{\bar{x}} & 0
\end{array}\right)^{(0)}\left(\vec{\psi}^{\theta}\right)^{(0)}
\end{aligned}
$$

where we have defined

$$
\mathcal{R}_{\bar{m}}=\left\{\left\langle A_{x}\right\rangle,\left[F_{y \bar{m}}^{\theta}, \cdot\right]\right\}-\left\{\left\langle A_{y}\right\rangle,\left[F_{x \bar{m}}^{\theta}, \cdot\right]\right\}
$$

and made use of the fact that $\left[\partial_{\bar{n}}, \mathcal{Q}\right]=-\mathcal{Q}_{\bar{n}}$.

To show that (4.28) is equivalent to (4.8), (4.11) notice that they can be rewritten as

$$
\bar{\partial}_{\mathbf{A}}\left(\vec{\psi}^{\theta}\right)^{(1)}=-\theta(\tilde{\mathbf{K}}+i \tilde{\mathbf{A}})^{(0)}\left(\vec{\psi}^{\theta}\right)^{(0)}
$$

and so one just needs to show that the elements of $-(\tilde{\mathbf{K}}+i \tilde{\mathbf{A}})^{(0)}$ and of the matrix in (4.28) are the same. For this one needs to notice that

$$
\mathcal{K}_{\bar{m}}+i \mathcal{A}_{\bar{m}}=\mathcal{Q}_{\bar{m}}+i \mathcal{R}_{\bar{m}}
$$

where $\mathcal{K}_{\bar{m}}$ and $\mathcal{A}_{\bar{m}}$ are defined as in (4.11). Finally, we have that ${ }^{11}$

$$
\mathcal{K}_{\bar{n}}+\frac{i}{2} \mathcal{A}_{\bar{n}}=\mathcal{Q}_{\bar{n}} \quad n=x, y
$$

\footnotetext{
${ }^{11} \operatorname{In}(4.32)$ we are using that, whenever $[A, B]=0$ we have the identity

$$
\{A,[B, C]\}-\frac{1}{2}[A B, C]=\frac{1}{2}(B C A-A C B)
$$

and that the rhs of this equation vanishes if $A$ and $B$ differ by multiplication of a function.
} 
whenever $\left\langle A_{x}\right\rangle^{(0)}$ and $\left\langle A_{y}\right\rangle^{(0)}$ are proportional matrices, which should be the case if the D-term equation (2.4) is to be satisfied for some choice of Kähler parameters. ${ }^{12}$

We have then shown that, upon the change of variables (4.22) the zero mode equations for non-commutative fields $\left(\hat{a}_{\bar{m}}, \hat{\varphi}_{x y}\right)$ is mapped to the $\beta$-deformed zero mode equations for the standard commutative fields $\left(a_{\bar{m}}, \varphi_{x y}\right)$. One may, in addition, show that the Yukawa couplings deduced in both formalisms match. In particular, this can be deduced from the results of appendix C.2, where the equivalence of the $8 \mathrm{~d}$ commutative and non-commutative formalisms is shown at the level of the superpotential. In the following we will focus on the computation of wavefunctions and Yukawa couplings in the non-commutative formalism which, as we will see, is particularly useful for deducing some important features of Yukawa couplings in intersecting 7-brane models.

\section{Wavefunctions and Yukawas in the non-commutative formalism}

In this section we discuss the equations of motion that follow from the non-perturbative superpotential $\hat{W}$ in (3.52). Our main motivation is to use the zero mode solutions to determine the $\theta$ corrections to the Yukawa couplings due to the trilinear term in $\hat{W}$.

In the previous section we have explained that the background values for the noncommutative fields $\langle\hat{A}\rangle$ and $\left\langle\hat{\Phi}_{x y}\right\rangle$ are equal to those of the commutative fields at zeroth order in $\epsilon$. More precisely, it is possible to take the holomorphic gauge in which $\left\langle\hat{A}_{\bar{m}}\right\rangle=0$, for $\bar{m}=\bar{x}, \bar{y}$, and therefore the equations of motion for the background imply that $\left\langle\hat{\Phi}_{x y}\right\rangle$ is holomorphic. The equations of motion for the fluctuations will then take a simpler form. In particular, in the holomorphic gauge the F-term equations for the fluctuations are given in equations (3.56). In addition there is a D-term which is the non-commutative extension of (2.4) [16]. Expanding the non-commutative wedge product according to the prescription in [16], and defining the fluctuations as in (3.55), yields the D-term equation

$$
\omega \wedge \partial_{\langle\hat{A}\rangle} \hat{a}-\frac{1}{2}[\langle\hat{\bar{\Phi}}\rangle, \hat{\varphi}]=0
$$

Notice that this equation does not receive $\mathcal{O}(\epsilon)$ corrections.

In the following we will use the $\mathrm{U}(3)$ toy model in order to carry out explicit calculations in the non-commutative approach. With the resulting zero modes we will also check that the non-commutative wave functions are related to the corrected wave functions obtained in appendix B via the dictionary established in (4.23).

\subsection{Non-commutative zero modes in the U(3) toy model}

The background value for $\left\langle\hat{\Phi}_{x y}\right\rangle$ is given exactly by (2.12). Just as in the commutative case the non zero vev breaks $\mathrm{U}(3)$ to $\mathrm{U}(1)^{3}$, with full enhancement occurring at the point of intersection of the three curves $\Sigma_{a}=\{x=0\}, \Sigma_{b}=\{y=0\}$, and $\Sigma_{c}=\{x=y\}$.

\footnotetext{
${ }^{12}$ Indeed, note that for our assumptions $\left[\left\langle A_{x}\right\rangle,\left\langle A_{y}\right\rangle\right]=0$ and $[\langle\Phi\rangle,\langle\bar{\Phi}\rangle]=0$ the D-term equation $(2.4)$ reduces to $\omega_{y \bar{y}} \partial_{\bar{x}}\left\langle A_{x}\right\rangle+\omega_{x \bar{x}} \partial_{\bar{y}}\left\langle A_{y}\right\rangle=0$ at the level of the background. The matrices $\left\langle A_{x}\right\rangle$ and $\left\langle A_{x}\right\rangle$ are clearly proportional if the D-term equation is satisfied. Changing the Kähler moduli of the compactification can be understood as changing the value of $\omega_{m \bar{m}}$, which will not change the fact that $\left\langle A_{x}\right\rangle^{(0)}$ and $\left\langle A_{y}\right\rangle^{(0)}$ must be proportional.
} 
Associated to each curve $\Sigma_{\alpha}$ there is a different set of fluctuations for which we use the notation

$$
\hat{\vec{\psi}}_{(\alpha)}=\left(\begin{array}{c}
\hat{\psi}_{\alpha \bar{x}} \\
\hat{\psi}_{\alpha \bar{y}} \\
\hat{\chi}_{\alpha}
\end{array}\right) \mathfrak{t}_{\alpha}=\hat{\vec{\psi}}_{\alpha} \mathfrak{t}_{\alpha}
$$

For simplicity we write $\chi=\chi_{x y}$. We are using the fermionic fluctuations which satisfy the same equations as their supersymmetric partners $\left(\hat{a}_{\alpha \bar{x}}, \hat{a}_{\alpha \bar{y}}, \hat{\varphi}_{\alpha x y}\right)$.

To obtain a $4 \mathrm{~d}$ chiral model we turn on a worldvolume flux of the form (2.17). We then choose the holomorphic gauge in which

$$
\langle\hat{A}\rangle=-\frac{i}{3}\left(M_{x} \bar{x} d x+M_{y} \bar{y} d y\right) \operatorname{diag}(1,-2,1)
$$

exactly as in (2.28). As in the commutative case we take $M_{x}<0<M_{y}$, so that the normalizable zero modes appear in the $a^{+}, b^{+}$and $c^{+}$sectors. The corresponding $\mathfrak{t}_{\alpha}$ generators for each curve are those given in (2.37).

The F-term equation $(3.56 \mathrm{~b})$ arising from $\hat{F}^{(0,2)}=0$ takes the same form in all sectors and will not be written separately. To first order in $\epsilon$ it implies that $\bar{\partial}_{\bar{x}} \hat{\psi}_{\alpha \bar{y}}=\bar{\partial}_{\bar{y}} \hat{\psi}_{\alpha \bar{x}}$, which will indeed be satisfied by the zero modes determined below.

With the main ingredients at hand we next proceed to obtain the zero modes in each sector. We will work out in more detail the case of $\theta$ constant. In section 5.1.1 we will briefly discuss an example with $\theta$ depending on the coordinates.

\section{Sector $a^{+}$}

Given the generator $\mathfrak{t}_{a^{+}}$it is straightforward to evaluate the various commutators and anticommutators in the F-term equations (3.56a). For generic $\theta$ we find

$$
\begin{aligned}
\bar{\partial}_{\bar{m}} \hat{\chi} & -i m_{\Phi}^{2} x \hat{\psi}_{\bar{m}}-\frac{\epsilon m_{\Phi}^{2}}{6}\left[\theta+\partial_{x} \theta\left(x-2 y-2 \Phi_{0}\right)\right] \partial_{y} \hat{\psi}_{\bar{m}} \\
& -\frac{\epsilon m_{\Phi}^{2}}{6}\left[2 \theta-\partial_{y} \theta\left(x-2 y-2 \Phi_{0}\right)\right] \partial_{x} \hat{\psi}_{\bar{m}}=\mathcal{O}\left(\epsilon^{2}\right)
\end{aligned}
$$

for $\bar{m}=\bar{x}, \bar{y}$. We have dropped the subindex $\alpha=a^{+}$that will be reinserted at the end. The D-term equation derived from (5.1) turns out to be

$$
\left(\partial_{x}-M_{x} \bar{x}\right) \hat{\psi}_{\bar{x}}+\left(\partial_{y}-M_{y} \bar{y}\right) \hat{\psi}_{\bar{y}}+i m_{\Phi}^{2} \bar{x} \hat{\chi}=0
$$

Notice that the worldvolume fluxes $M_{x}$ and $M_{y}$ only appear in the D-term equation. In practice this feature greatly simplifies the search of zero mode solutions. More importantly, it makes clear from the beginning that the Yukawa couplings, which only depend on the F-terms, will turn out to be independent of fluxes [16].

To find the zero modes we make an Ansatz motivated by the form of the solutions when $\theta=0$ collected in eq. (2.38). In particular we set $\hat{\psi}_{\bar{y}}=0$, which then implies $\bar{\partial}_{\bar{y}} \hat{\psi}_{\bar{x}}=\bar{\partial}_{\bar{y}} \hat{\chi}=0$. To solve the remaining equations we have to treat separately the cases of $\theta$ constant and $\theta$ coordinate dependent. 
When $\theta$ is constant we further impose

$$
\hat{\psi}_{\bar{x}}=-\frac{i \lambda_{a}}{m_{\Phi}^{2}} \hat{\chi} \quad ; \quad \hat{\chi}=e^{\lambda_{a}|x|^{2}} G_{a}(y, \bar{x})
$$

In this way the D-term equation is satisfied with $\lambda_{a}=\lambda_{a}^{-}$, defined in (2.25). The root $\lambda_{a}^{+}$is discarded because it yields zero modes that are not localized on the curve $x=0$. It remains to solve the F-term (5.4). Inserting the Ansatz for $\hat{\psi}_{\bar{x}}$ and $\hat{\chi}$ gives an equation for $G_{a}(\bar{x}, y)$ that is easily solved to first order in $\epsilon$. The final result can be written as

$$
\hat{\vec{\psi}}_{a^{+}}^{i}=\left(\begin{array}{c}
-\frac{i \lambda_{a}}{m_{\Phi}^{2}} \\
0 \\
1
\end{array}\right) \hat{\chi}_{a^{+}}^{i} \quad ; \quad \hat{\chi}_{a^{+}}^{i}=e^{\lambda_{a}|x|^{2}}\left(f_{i}(y)-\frac{i \epsilon \theta}{6} \lambda_{a}^{2} \bar{x}^{2} f_{i}(y)-\frac{i \epsilon \theta}{6} \lambda_{a} \bar{x} f_{i}^{\prime}(y)\right)
$$

where $f_{i}(y)$ is an arbitrary holomorphic function, and $i$ labels the different zero modes.

It is interesting to compare the non-commutative wavefunctions with those obtained in appendix B within a commutative approach. In sector $a^{+}$we can read $\chi_{a^{+}}^{i}$ from (B.21) after applying the operators $\mathcal{D}_{x}$ and $\mathcal{D}_{y}$ as indicated. We find

$$
\chi_{a^{+}}^{i}=e^{\lambda_{a}|x|^{2}}\left\{f_{i}(y)-\frac{i \epsilon \theta}{6} \lambda_{a}^{2} \bar{x}^{2} f_{i}(y)-\frac{i \epsilon \theta}{6} \bar{x}\left[\left(\lambda_{a}+M\right) f_{i}^{\prime}(y)+M \lambda_{a} \bar{y} f_{i}(y)\right]\right\}
$$

where we used that $\psi_{0, a^{+}}=e^{\lambda_{a}|x|^{2}} f_{i}(y)$. To first order in $\epsilon$ we see that the difference with (5.7) is given by

$$
\tilde{\chi}_{a^{+}}^{i}=\hat{\chi}_{a^{+}}^{i}-\chi_{a^{+}}^{i}=\frac{i \epsilon \theta}{6} M\left(\bar{x} \partial_{y} \chi_{a^{+}}^{i}+\bar{y} \partial_{x} \chi_{a^{+}}^{i}\right)
$$

For $\theta$ constant this is precisely the result expected from the map established in (4.23b). Finally, notice that $\psi_{a^{+} \bar{x}}^{i}$ and $\hat{\psi}_{a^{+} \bar{x}}^{i}$ are also correctly related.

\section{Sector $b^{+}$}

In this sector the F-term equations (3.56a) reduce to

$$
\begin{aligned}
\bar{\partial}_{\bar{m}} \hat{\chi} & +i m_{\Phi}^{2} y \hat{\psi}_{\bar{m}}+\frac{\epsilon m_{\Phi}^{2}}{6}\left[\theta-\partial_{y} \theta\left(2 x-y+2 \Phi_{0}\right)\right] \partial_{x} \hat{\psi}_{\bar{m}} \\
& +\frac{\epsilon m_{\Phi}^{2}}{6}\left[2 \theta+\partial_{x} \theta\left(2 x-y+2 \Phi_{0}\right)\right] \partial_{y} \hat{\psi}_{\bar{m}}=\mathcal{O}\left(\epsilon^{2}\right)
\end{aligned}
$$

for $\bar{m}=\bar{x}, \bar{y}$. The $\mathrm{D}$-term equation is given by

$$
\left(\partial_{x}+M_{x} \bar{x}\right) \hat{\psi}_{\bar{x}}+\left(\partial_{y}+M_{y} \bar{y}\right) \hat{\psi}_{\bar{y}}-i m_{\Phi}^{2} \bar{y} \hat{\chi}=0
$$

The subindex $\alpha=b^{+}$will be omitted until the final result. Now it is consistent to set $\hat{\psi}_{\bar{x}}=0$, which then requires $\bar{\partial}_{\bar{x}} \hat{\psi}_{\bar{y}}=\bar{\partial}_{\bar{x}} \hat{\chi}=0$.

Our previous results for $\theta$ constant suggest the Ansatz

$$
\hat{\psi}_{\bar{y}}=\frac{i \lambda_{b}}{m_{\Phi}^{2}} \hat{\chi} \quad ; \quad \hat{\chi}=e^{\lambda_{b}|y|^{2}} G_{b}(x, \bar{y})
$$


The D-term equation is then verified with $\lambda_{b}=\lambda_{b}^{-}$, defined in (2.33). The auxiliary function $G_{b}$ is determined to first order in $\epsilon$ substituting the Ansatz in the F-term (5.10). In the end we obtain

$$
\hat{\vec{\psi}}_{b^{+}}^{j}=\left(\begin{array}{c}
0 \\
\frac{i \lambda_{b}}{m_{\Phi}^{2}} \\
1
\end{array}\right) \hat{\chi}_{b^{+}}^{j} \quad ; \quad \hat{\chi}_{b^{+}}^{j}=e^{\lambda_{b}|y|^{2}}\left(g_{j}(x)-\frac{i \epsilon \theta}{6} \lambda_{b}^{2} \bar{y}^{2} g_{j}(x)-\frac{i \epsilon \theta}{6} \lambda_{b} \bar{y} g_{j}^{\prime}(x)\right)
$$

with $j$ indexing different zero modes.

In this sector the commutative wavefunction $\chi_{b^{+}}^{j}$ obtained from (B.29) reads

$$
\chi_{b^{+}}^{j}=e^{\lambda_{b}|y|^{2}}\left\{g_{j}(x)-\frac{i \epsilon \theta}{6} \lambda_{b}^{2} \bar{y}^{2} g_{j}(x)-\frac{i \epsilon \theta}{6} \bar{y}\left[\left(\lambda_{b}+M\right) g_{j}^{\prime}(x)+M \lambda_{b} \bar{x} g_{j}(x)\right]\right\}
$$

where we took $\psi_{0, b^{+}}=e^{\lambda_{b}|y|^{2}} g_{j}(x)$. It is easy to check that $\hat{\chi}_{b^{+}}^{j}$ is recovered after adding $\tilde{\chi}_{b^{+}}^{j}$ computed according to $(4.23 \mathrm{~b})$.

\section{Sector $c^{+}$}

Substituting the fluctuations and the vevs in (3.56a) yields the F-term equations

$$
\begin{aligned}
\bar{\partial}_{\bar{m}} \hat{\chi} & +i m_{\Phi}^{2}(x-y) \hat{\psi}_{\bar{m}}+\frac{\epsilon m_{\Phi}^{2}}{6}\left[\theta+\partial_{y} \theta\left(x+y-2 \Phi_{0}\right)\right] \partial_{x} \hat{\psi}_{\bar{m}} \\
& -\frac{\epsilon m_{\Phi}^{2}}{6}\left[\theta+\partial_{x} \theta\left(x+y-2 \Phi_{0}\right)\right] \partial_{y} \hat{\psi}_{\bar{m}}=\mathcal{O}\left(\epsilon^{2}\right)
\end{aligned}
$$

for $\bar{m}=\bar{x}, \bar{y}$. As before the subindex $\alpha=c^{+}$is dropped. The D-term equation takes the simple form

$$
\partial_{x} \hat{\psi}_{\bar{x}}+\partial_{y} \hat{\psi}_{\bar{y}}-i m_{\Phi}^{2}(\bar{x}-\bar{y}) \hat{\chi}=0
$$

In this case the condition $\hat{\psi}_{\bar{x}}=-\hat{\psi}_{\bar{y}}$ can be imposed consistently with (3.56b).

For $\theta$ constant the Ansatz inspired by known results is now

$$
\hat{\psi}_{\bar{x}}=-\hat{\psi}_{\bar{y}}=\frac{i \lambda_{c}}{m_{\Phi}^{2}} \hat{\chi} \quad ; \quad \hat{\chi}=e^{\lambda_{c}|x-y|^{2}} G_{c}(\bar{x}, \bar{y})
$$

Inserting the Ansatz in the equations shows that it works with $\lambda_{c}$ given in (2.33). Summarizing we obtain

$$
\hat{\vec{\psi}}_{c^{+}}=\left(\begin{array}{c}
\frac{i \lambda_{c}}{m_{\Phi}^{2}} \\
-\frac{i \lambda_{c}}{m_{\Phi}^{2}} \\
1
\end{array}\right) \hat{\chi}_{c^{+}} \quad ; \quad \hat{\chi}_{c^{+}}=\gamma_{c} m_{*} e^{\lambda_{c}|x-y|^{2}}\left(1-\frac{i \epsilon \theta}{6} \lambda_{c}^{2}(\bar{x}-\bar{y})^{2}\right)
$$

To determine $G_{c}$ we assumed that it is constant at lowest order in $\epsilon$. The reason is that at $\Sigma_{c}$ there is only one set of zero modes corresponding to the Higgs. The constant is taken to be $\gamma_{c} m_{*}$, where $\gamma_{c}$ is an adimensional normalization factor.

The commutative wavefunction $\chi_{c^{+}}$computed from (B.36), using $\psi_{0, c^{+}}=\gamma_{c} m_{*} e^{\lambda_{c}|x-y|^{2}}$, is given by

$$
\chi_{c^{+}}=\gamma_{c} m_{*} e^{\lambda_{c}|x-y|^{2}}\left[1-\frac{2 i \epsilon \theta}{3} \lambda_{c}^{2}(\bar{x}-\bar{y})^{2}\left(\frac{1}{4}+\frac{M}{2 \lambda_{c}}\right)\right]
$$

There is again perfect matching with $\hat{\chi}_{c^{+}}$after the map (4.23b). 


\subsubsection{Zero modes with $\theta$ coordinate dependent}

As we will see, with $\theta$ constant it is not possible to generate a realistic pattern for the Yukawa couplings. This motivates us to consider a more general case with $\theta$ coordinate dependent. We have been able to find the zero modes in closed form when $\theta$ is the linear function

$$
\theta=\theta_{0}+\theta_{1} x+\theta_{2} y
$$

where the $\theta_{\ell}$ are constants. In this case it is no longer consistent to make an Ansatz such as (5.17) in which the non-zero $\hat{\psi}_{\alpha \bar{m}}$ are proportional to $\hat{\chi}_{\alpha}$.

To illustrate the strategy that works for the linear $\theta$ let us focus in the $a^{+}$sector. Again it is allowed to take $\hat{\psi}_{a^{+} \bar{y}}=0$. The new Ansatz for the non-trivial fluctuations consists of first setting

$$
\hat{\psi}_{a^{+} \bar{x}}=-\frac{i \lambda_{a}}{m_{\Phi}^{2}} e^{\lambda_{a}|x|^{2}} H_{a}(y, x, \bar{x})
$$

and then solving for $\hat{\chi}_{a^{+}}$from the D-term equation (5.5). The constant $\lambda_{a}$ is again equal to the $\lambda_{a}^{-}$defined in (2.25). It thus follows that

$$
\hat{\chi}_{a^{+}}=e^{\lambda_{a}|x|^{2}}\left(H_{a}+\frac{\lambda_{a}}{m_{\Phi}^{4} \bar{x}} \partial_{x} H_{a}\right)
$$

We also impose the condition that $\hat{\chi}_{a^{+}} \rightarrow f_{i}(y)$ when $x \rightarrow 0$. To determine the function $H_{a}$ we substitute the above $\hat{\psi}_{a^{+} \bar{x}}$ and $\hat{\chi}_{a^{+}}$into the F-term (5.4) and solve to first order in $\epsilon$. In this way we find

$$
\begin{aligned}
H_{a} & =f_{i}(y)+\epsilon \theta_{0} \bar{x} \alpha_{0}+\epsilon \theta_{1}\left(\bar{x} \alpha_{1}+\frac{i \lambda_{a}^{2}}{3\left(\lambda_{a}^{2}+m_{\Phi}^{4}\right)} f_{i}^{\prime}(y)\right)+\epsilon \theta_{2} \bar{x} \alpha_{2} \\
\alpha_{0} & =-\frac{i}{6} \lambda_{a}^{2} \bar{x} f_{i}(y)-\frac{i}{6} \lambda_{a} f_{i}^{\prime}(y) \\
\alpha_{1} & =\frac{i \lambda_{a}^{2}}{3\left(\lambda_{a}^{2}+2 m_{\Phi}^{4}\right)}\left(\lambda_{a}-m_{\Phi}^{4} x \bar{x}\right) f_{i}(y)-\frac{i \lambda_{a}}{3}\left(\frac{m_{\Phi}^{4}}{\left(\lambda_{a}^{2}+m_{\Phi}^{4}\right)} x-y-\Phi_{0}\right) f_{i}^{\prime}(y) \\
\alpha_{2} & =-\frac{i \lambda_{a}}{6} y f_{i}^{\prime}(y)-\frac{i \lambda_{a}^{2}}{6}\left(\frac{\lambda_{a}-m_{\Phi}^{4} x \bar{x}}{\left(\lambda_{a}^{2}+2 m_{\Phi}^{4}\right)}+2 y \bar{x}+\Phi_{0} \bar{x}\right) f_{i}(y)
\end{aligned}
$$

Notice that for $\theta$ constant we recover our previous result (5.7). We remark that the F-term equations are satisfied to $\mathcal{O}(\epsilon)$ for any $\lambda_{a}$. Hence, we expect the $\lambda_{a}$ dependence to drop out completely in the computation of Yukawa couplings.

The wavefunctions in the $b^{+}$sector can be found in a similar fashion. We start with $\hat{\psi}_{b^{+} \bar{x}}=0$, together with

$$
\hat{\psi}_{b^{+} \bar{y}}=\frac{i \lambda_{b}}{m_{\Phi}^{2}} e^{\lambda_{b}|y|^{2}} H_{b}(x, y, \bar{y})
$$

where $\lambda_{b}$ is equal to $\lambda_{b}^{-}$defined in (2.33). The corresponding $\hat{\chi}_{b^{+}}$is such that the D-term equation (5.11) is verified and is required to satisfy $\hat{\chi}_{b^{+}} \rightarrow g_{j}(x)$ when $y \rightarrow 0$. The function $H_{b}$ is determined from the F-term eq. (5.10). To order $\epsilon$ it is given by

$$
H_{b}=g_{j}(x)+\epsilon \theta_{0} \bar{y} \beta_{0}+\epsilon \theta_{1} \bar{y} \beta_{1}+\epsilon \theta_{2}\left(\bar{y} \beta_{2}+\frac{i \lambda_{b}^{2}}{3\left(\lambda_{b}^{2}+m_{\Phi}^{4}\right)} g_{j}^{\prime}(x)\right)
$$


The $\beta_{\ell}$ are obtained from the $\alpha_{\ell}$ in eq. (5.23) as $\beta_{0}=\alpha_{0}, \beta_{1}=\alpha_{2}$, and $\beta_{2}=\alpha_{1}$, upon the exchanges $x \rightarrow y, \lambda_{a} \rightarrow \lambda_{b}$, and $f_{i}(y) \rightarrow g_{j}(x)$. The F-term equations do not constrain the value of $\lambda_{b}$.

In the $c^{+}$sector we take

$$
\hat{\psi}_{c^{+} \bar{x}}=\frac{i \lambda_{c}}{m_{\Phi}^{2}} e^{\lambda_{c}|x-y|^{2}} H_{c}(x, \bar{x}, y, \bar{y})=-\hat{\psi}_{c^{+}} \bar{y}
$$

where $\lambda_{c}=-m_{\Phi}^{2} / \sqrt{2}$. From the D-term equation (5.16) $\hat{\chi}_{c^{+}}$is then determined to be

$$
\hat{\chi}_{c^{+}}=e^{\lambda_{c}|x-y|^{2}}\left(H_{c}+\frac{\lambda_{c}}{m_{\Phi}^{4}} \frac{\partial_{x} H_{c}-\partial_{y} H_{c}}{\bar{x}-\bar{y}}\right)
$$

For $H_{c}$ we make the Ansatz

$$
H_{c}=m_{*} \gamma_{c}\left[1+\epsilon(\bar{x}-\bar{y})\left(\theta_{0} \nu_{0}+\theta_{1} \nu_{1}+\theta_{2} \nu_{2}\right)\right]
$$

and deduce the $\nu_{\ell}$ substituting in the F-term eqs. (5.15). This procedure yields

$$
\begin{aligned}
& \nu_{0}=-\frac{i}{6} \lambda_{c}^{2}(\bar{x}-\bar{y}) \\
& \nu_{1}=\frac{i \lambda_{c}^{2}}{12\left(\lambda_{c}^{2}+m_{\Phi}^{4}\right)}\left\{2 \lambda_{c}-(\bar{x}-\bar{y})\left[\left(2 \lambda_{c}^{2}+3 m_{\Phi}^{4}\right) x+\left(2 \lambda_{c}^{2}+m_{\Phi}^{4}\right) y-2\left(\lambda_{c}^{2}+m_{\Phi}^{4}\right) \Phi_{0}\right]\right\} \\
& \nu_{2}=-\frac{i \lambda_{c}^{2}}{12\left(\lambda_{c}^{2}+m_{\Phi}^{4}\right)}\left\{2 \lambda_{c}+(\bar{x}-\bar{y})\left[\left(2 \lambda_{c}^{2}+3 m_{\Phi}^{4}\right) x+\left(2 \lambda_{c}^{2}+m_{\Phi}^{4}\right) y-2\left(\lambda_{c}^{2}+m_{\Phi}^{4}\right) \Phi_{0}\right]\right\}
\end{aligned}
$$

These solutions can be simplified inserting the actual value of $\lambda_{c}$. However, the F-term equations are satisfied for generic $\lambda_{c}$.

\subsection{Non-commutative Yukawa couplings}

The starting point is the $\epsilon$ expansion of the trilinear superpotential $\hat{W}_{\text {Yuk }}$ given in eq. (3.58). To compute the couplings we further need to substitute the zero modes collected in the vector $\hat{\vec{\psi}}_{\alpha}$. From $\hat{W}_{0}$ there is a coupling $Y_{0}$ that is given by $(2.36)$ replacing $\vec{\psi}_{\alpha}$ by $\hat{\vec{\psi}}_{\alpha}$. From $\hat{W}_{1}$ in (3.60) we obtain a contribution

$$
\begin{aligned}
\left(Y_{1}\right)_{a b c}^{i j k} & =-m_{*} d_{a b c} \int_{S} \theta\left(\partial_{x} \hat{\psi}_{a \bar{x}}^{i} \partial_{y} \hat{\psi}_{b \bar{y}}^{j}-\partial_{y} \hat{\psi}_{a \bar{x}}^{i} \partial_{x} \hat{\psi}_{b \bar{y}}^{j}-\partial_{x} \hat{\psi}_{a \bar{y}}^{i} \partial_{y} \hat{\psi}_{b \bar{x}}^{j}+\partial_{y} \hat{\psi}_{a \bar{y}}^{i} \partial_{x} \hat{\psi}_{b \bar{x}}^{j}\right) \hat{\chi}_{c}^{k} d \operatorname{vol}_{S} \\
& + \text { cyclic permutations in } a, b, c
\end{aligned}
$$

Both pieces $Y_{0}$ and $Y_{1}$ have an $\epsilon$ expansion since the zero modes can be written as $\hat{\vec{\psi}}=\hat{\vec{\psi}}^{(0)}+\epsilon \hat{\vec{\psi}}^{(1)}+\mathcal{O}\left(\epsilon^{2}\right)$. Notice that at zeroth order the non-commutative and commutative wavefunctions do coincide and in fact $\hat{\vec{\psi}}^{(0)}=\vec{\psi}^{(0)}$. Taking into account the expansion of $\hat{W}_{\text {Yuk }}$ we see that indeed the Yukawa couplings have the schematic structure

$$
Y=Y_{0}^{(0)}+\epsilon\left(Y_{0}^{(1)}+Y_{1}^{(0)}\right)+\mathcal{O}\left(\epsilon^{2}\right)
$$

where we have omitted indices for simplicity. Here $Y_{0}^{(0)}$ and $Y_{0}^{(1)}$ both originate from $Y_{0}$. More concretely, $Y_{0}^{(0)}$ is computed from $(2.36)$ replacing $\vec{\psi}_{\alpha}^{i}$ by $\left(\vec{\psi}_{\alpha}^{i}\right)^{(0)}$, whereas $Y_{0}^{(1)}$ is given by (3.50) with $\vec{\psi}_{\alpha}^{i}$ replaced by $\hat{\vec{\psi}}_{\alpha}^{i}$. On the other hand, the $\mathcal{O}(\epsilon)$ contribution $Y_{1}^{(0)}$ is computed inserting the uncorrected wavefunctions $\left(\vec{\psi}_{\alpha}^{i}\right)^{(0)}$ in (5.30). 


\section{Corrected Yukawa couplings in the U(3) model}

The calculation of the Yukawa couplings corrected to $\mathcal{O}(\epsilon)$ can be implemented in the $\mathrm{U}(3)$ model using the wavefunctions determined in section 5.1. As before, we take the Higgs to arise from the curve $\Sigma_{c}$, whereas the quark and lepton families come from the curves $\Sigma_{a}$ and $\Sigma_{b}$, and are indexed by $i$ and $j$ respectively. The full Yukawa couplings are denoted by $Y^{i j}$, and the two types of contributions by $Y_{0}^{i j}$ and $Y_{1}^{i j}$. For the holomorphic functions appearing in the wavefunctions we again adopt the basis of [13] in which $f_{i}(y)=\gamma_{a i} m_{*}^{4-i} y^{3-i}$ and $g_{j}(x)=\gamma_{b j} m_{*}^{4-j} x^{3-j}$, with $i, j=1,2,3$. The normalization factors $\gamma_{a i}$ and $\gamma_{b j}$ will be specified later.

We will first determine the couplings when $\theta$ is constant. The calculation simplifies considerably because the wavefunctions $\hat{\psi}_{\alpha \bar{m}}$ are either zero or are proportional to $\hat{\chi}_{\alpha}$ as shown in equations (5.6), (5.12) and (5.17). From section 2.4 we already know that $\left(Y_{0}^{(0)}\right)^{i j}$ is zero for $i \neq 3, j \neq 3$, and $\left(Y_{0}^{(0)}\right)^{33}=-i \pi^{2} \gamma_{a 3} \gamma_{b 3} \gamma_{c} m_{*}^{4} / m_{\Phi}^{4}$. We will then concentrate on the pieces $Y_{0}^{(1)}$ and $Y_{1}^{(0)}$ for which we can write down explicit expressions using the properties of the wavefunctions derived in section 5.1.

Inserting the known wavefunctions in $(3.50)$ we obtain the $\mathcal{O}(\epsilon)$ contribution from $\hat{W}_{0}$

$$
\begin{aligned}
\left(Y_{0}^{(1)}\right)^{i j}= & -\frac{\theta m_{*}^{2} \gamma_{c}}{6 m_{\Phi}^{4}}\left(\lambda_{a} \lambda_{b}+\lambda_{a} \lambda_{c}+\lambda_{b} \lambda_{c}\right) I_{0}^{i j} \\
I_{0}^{i j}= & \int_{S}\left\{f_{i}(y) g_{j}(x)\left[\lambda_{c}^{2}(\bar{x}-\bar{y})^{2}+\lambda_{a}^{2} \bar{x}^{2}+\lambda_{b}^{2} \bar{y}^{2}\right]\right. \\
& \left.+\left[\lambda_{a} \bar{x} f_{i}^{\prime}(y) g_{j}(x)+\lambda_{b} \bar{y} f_{i}(y) g_{j}^{\prime}(x)\right]\right\} e^{\lambda_{a}|x|^{2}+\lambda_{b}|y|^{2}+\lambda_{c}|x-y|^{2}} d \operatorname{vol}_{S}
\end{aligned}
$$

Substituting in (5.30) yields the $\mathcal{O}(\epsilon)$ contribution from $\hat{W}_{1}$

$$
\begin{aligned}
\left(Y_{1}^{(0)}\right)^{i j}= & -\frac{m_{*}^{2} \gamma_{c}}{2 m_{\Phi}^{4}} I_{1}^{i j} \\
I_{1}^{i j}= & \int_{S} \theta\left\{\lambda_{c}^{2}(\bar{x}-\bar{y})\left[\left(\lambda_{a}^{2} \bar{x}-\lambda_{b}^{2} \bar{y}\right) f_{i}(y) g_{j}(x)+\lambda_{a} f_{i}^{\prime}(y) g_{j}(x)-\lambda_{b} f_{i}(y) g_{j}^{\prime}(x)\right]\right. \\
& \left.+\lambda_{a} \lambda_{b}\left[\lambda_{a} \lambda_{b} \bar{x} \bar{y} f_{i}(y) g_{j}(x)-f_{i}^{\prime}(y) g_{j}^{\prime}(x)\right]\right\} e^{\lambda_{a}|x|^{2}+\lambda_{b}|y|^{2}+\lambda_{c}|x-y|^{2}} d \operatorname{vol}_{S}
\end{aligned}
$$

This formula is also valid when $\theta$ is coordinate dependent. Here we have used that in the $\mathrm{U}(3)$ model $d_{a^{+} b^{+} c^{+}}=\mathrm{S} \operatorname{Tr}\left(\mathfrak{t}_{a^{+}}, \mathfrak{t}_{b+}, \mathfrak{t}_{c^{+}}\right)=\frac{1}{2}$.

The coupling $Y^{33}$ does not receive $\mathcal{O}(\epsilon)$ corrections. Indeed, the integrals appearing in $\left(Y_{0}^{(1)}\right)^{33}$ and $\left(Y_{1}^{(0)}\right)^{33}$ vanish because the product of wavefunctions in the integrand is not invariant under the diagonal $\mathrm{U}(1)$ rotation $x \rightarrow e^{i \alpha} x$ and $y \rightarrow e^{i \alpha} y$. By the same token we also conclude that for constant $\theta$ only the couplings $Y^{22}, Y^{31}$, and $Y^{13}$ can be different from zero.

To evaluate the integrals we extend $|x|$ and $|y|$ to infinity as we did in absence of $\theta$ corrections. After a simple calculation we obtain the couplings

$$
Y^{22}=\frac{\epsilon \pi^{2} \theta m_{*}^{6}}{3 m_{\Phi}^{4}} \gamma_{a 2} \gamma_{b 2} \gamma_{c} \quad ; \quad Y^{31}=-\frac{\epsilon \pi^{2} \theta m_{*}^{6}}{3 m_{\Phi}^{4}} \gamma_{a 3} \gamma_{b 1} \gamma_{c} \quad ; \quad Y^{31}=-\frac{\epsilon \pi^{2} \theta m_{*}^{6}}{3 m_{\Phi}^{4}} \gamma_{a 1} \gamma_{b 3} \gamma_{c}
$$


We find exactly the same couplings following the commutative approach, using the wavefunctions computed in appendix B. Observe that, up to normalization, the couplings are independent of worldvolume flux, in agreement with the general result of [16].

We have also found the couplings for perturbations linear in the local coordinates, i.e.

$$
\theta=3 i\left(\theta_{0}+\theta_{1} x+\theta_{2} y\right)
$$

where the $3 i$ factor is added to simplify the results. Inserting the wavefunctions given in section 5.1.1 and evaluating the integrals leads to the Yukawa matrix

$$
\frac{Y}{Y^{33}}=\left(\begin{array}{ccc}
\mathcal{O}\left(\epsilon^{2}\right) & \mathcal{O}\left(\epsilon^{2}\right) & \epsilon m_{*}^{2} \frac{\gamma_{a 1}}{\gamma_{a 3}}\left(\theta_{0}+\theta_{1} \Phi_{0}\right) \\
\mathcal{O}\left(\epsilon^{2}\right) & \epsilon m_{*}^{2} \frac{\gamma_{a 2} \gamma_{b 2}}{\gamma_{a 3} \gamma_{b 3}}\left[\left(\theta_{1}+\theta_{2}\right) \Phi_{0}-\theta_{0}\right] & \epsilon m_{*} \frac{\gamma_{a 2}}{\gamma_{a 3}} \theta_{2} \\
\epsilon m_{*}^{2} \frac{\gamma_{b 1}}{\gamma_{b 3}}\left(\theta_{0}+\theta_{2} \Phi_{0}\right) & \epsilon m_{*} \frac{\gamma_{b 2}}{\gamma_{b 3}} \theta_{1} & 1
\end{array}\right)
$$

As expected, up to normalization the couplings are independent of worldvolume fluxes. We have obtained the same results using the residue techniques of [16] where a similar calculation was performed but only in the case $\theta_{0}=0$ and $\theta_{1}=-\theta_{2}$. In the next section we will discuss possible phenomenological implications of this type of Yukawa structure.

\section{Flux dependence, D-terms and Yukawa couplings}

\subsection{The $Y(D)=Y(L)$ problem}

It is interesting to see to what extent a simplified model like the $\mathrm{U}(3)$ example above with constant fluxes and linearly dependent perturbations can display a hierarchical structure which could be useful in a more realistic local SU(5) F-theory GUT. Setting $\gamma_{a i}=\gamma_{b i}=1$, one observes that the Yukawa matrix eq. (5.36) has eigenvalues of order 1, $\epsilon, \epsilon^{2}$ and hence has a promising structure to generate such mass hierarchies. However the Yukawa couplings computed above are flux independent. In an SU(5) local GUT the Yukawa couplings of D-quark and leptons come from couplings $\mathbf{1 0} \times \overline{\mathbf{5}} \times \overline{\mathbf{5}}_{\mathbf{H}}$ and before the addition of hypercharge fluxes one has identical Yukawa couplings for D-quarks and leptons of all three generations, i.e., $Y^{i j}(D)=Y^{i j}(L)$. Since eq. (5.36) is independent of fluxes this equality will persist even after the addition of hypercharge fluxes. These equalities are however not consistent with the measured quark and lepton masses of the first two generations. ${ }^{13}$ This seems to be a general problem of Yukawa couplings in local F-theory GUT models, and is ultimately connected to the existence of the holomorphic gauge discussed in previous sections. Using this holomorphic gauge the fluxes disappear from the local F-term equations and the resulting holomorphic Yukawas are flux independent.

\footnotetext{
${ }^{13}$ After renormalization group corrections one finds $m_{b}\left(M_{Z}\right) / m_{\tau}\left(M_{Z}\right) \simeq\left(\alpha_{3}\left(M_{Z}\right) / \alpha_{3}\left(M_{*}\right)\right)^{\gamma / 2 b_{3}} \simeq$ $\left(\alpha_{3}\left(M_{Z}\right) / \alpha_{3}\left(M_{*}\right)\right)^{8 / 9} \simeq 2.5$, in reasonable agreement with experiment. However for the lightest two generations such a prediction fails. Experimental results are better described by boundary conditions $m_{\mu} \simeq 3 m_{s}$ and $m_{e} \simeq m_{d} / 3$ at the unification scale. In standard GUT's getting these boundary conditions requires higher dimensional Higgs representations in a $\mathbf{4 5}$ of SU(5) [49] or else non-renormalizable interactions [50] involving the adjoint Higgs, i.e. $10 \times \Phi_{\mathbf{2} 4}^{n} \times \overline{\mathbf{5}} \times \overline{\mathbf{5}}_{H}$, both options using additional, poorly motivated, flavour symmetries.
} 


\subsection{Normalization and flux dependence in physical couplings}

There is however a relevant point we did not take into account yet. The wave functions we have used to compute the Yukawa couplings were not normalized as they should if one is to compare with physical quantities. To see the effect of normalization it will be sufficient for our purposes to compute the normalization of the wave functions in the absence of the perturbations. Let us consider the $a$ sector and to simplify let us impose the BPS condition on the fluxes, i.e. $M_{x}=M$ and $M_{y}=-M$. We take $M<0$ so that the normalizable zero modes are in the $a^{+}$sector. We need to use the wave functions in real gauge as given in eq. (2.27), i.e.

$$
\vec{\psi}_{a i}^{\text {real }}=\left(\begin{array}{c}
-\frac{i \lambda_{a}}{m_{\Phi}^{2}} \\
0 \\
1
\end{array}\right) \quad \chi_{a i}^{\text {real }}
$$

where $\lambda_{a}=\lambda_{a}^{-}$is defined in eq. (2.24) and the real scalar wavefunction is

$$
\chi_{a i}^{\text {real }}=e^{-\sqrt{\left(\frac{M}{2}\right)^{2}+m_{\Phi}^{4}}|x|^{2}} e^{-\frac{|M|}{2}|y|^{2}} f_{i}(y) .
$$

In the real gauge an exponential suppression along the $y$ coordinate is made explicit. The wave function is only sensitive to local physics and one can normalize the wave function without the addition of any volume dependent cut-off. To normalize the states we perform the integration

$$
\left\|\chi_{a i}^{\mathrm{real}}\right\|^{2}=\int_{S}\left|\chi_{a i}^{\mathrm{real}}\right|^{2} d \operatorname{vol}_{S}=\left|\gamma_{i}\right|^{2} \frac{\pi^{2}(3-i) !}{2 \sqrt{\left(\frac{M}{2}\right)^{2}+m_{\Phi}^{4}}\left(|M| / m_{*}^{2}\right)^{4-i}},
$$

where we have extended the integration along $|x|$ and $|y|$ to infinite radius as in the calculation of Yukawa couplings. The normalization condition amounts to imposing

$$
\left\langle\vec{\psi}_{a i}^{\mathrm{real}} \mid \vec{\psi}_{a j}^{\mathrm{real}}\right\rangle=m_{*}^{2} \int_{S} \operatorname{Tr}\left(\vec{\psi}_{a i}^{\mathrm{real}} \cdot \vec{\psi}_{a j}^{\dagger \text { real }}\right) \mathrm{dvol}_{S}=\delta_{i j}
$$

where the $\delta_{i j}$ structure arises because the exponentials in the wavefunctions, as well as the measure, are invariant under the diagonal $\mathrm{U}(1)$ rotation $x \rightarrow e^{i \theta} x$ and $y \rightarrow e^{i \theta} y$. Upon normalization we get

$$
\gamma_{a i}^{2}=\frac{\left(|M| / m_{*}^{2}\right)^{3-i}}{(3-i) !} \times \mathcal{N}_{a}^{-1}, \quad \mathcal{N}_{a}=\frac{\pi^{2} m_{*}^{4}}{2 m_{\Phi}^{4}}\left(1+\sqrt{1+\frac{4 m_{\Phi}^{4}}{|M|^{2}}}\right),
$$

where $\mathcal{N}_{a}$ is generation independent. Note that in the dilute flux limit $m_{*}, m_{\Phi} \gg|M|$ one has $\mathcal{N}_{a} \simeq m_{*}^{4} /\left(m_{\Phi}^{2}|M|\right)$. If the flux scales like $|M| \simeq 1 / R^{2}$ this correspond to a metric for the matter fields $K_{i i} \simeq 1 / R^{2}$. This is analogous to the modulus behavior of of the metrics of perturbative intersecting D7-brane models, see e.g. refs. [51, 52].

Similar results are obtained for the matter fields in curve $b$. In the case of the curve $c$ with the flux choice in section 2 there is no flux along the curve. In this case the exponential 
damping along the curve is missing and one has to take a volume dependent cut-off in order to normalize the wave function. However, we will not need to do so because in the Yukawa coupling ratios in eq. (5.36) the normalization of the c curve cancels out. Plugging these values for $\gamma_{a i}, \gamma_{b i}$ in eq. (5.36) one obtains a matrix

$$
\frac{Y}{Y^{33}}=\left(\begin{array}{ccc}
\mathcal{O}\left(\epsilon^{2}\right) & \mathcal{O}\left(\epsilon^{2}\right) & \frac{|M|}{\sqrt{2}} \epsilon\left(\theta_{0}+\theta_{1} \Phi_{0}\right) \\
\mathcal{O}\left(\epsilon^{2}\right) & |M| \epsilon\left[\left(\theta_{1}+\theta_{2}\right) \Phi_{0}-\theta_{0}\right] & |M|^{1 / 2} \epsilon \theta_{2} \\
\frac{|M|}{\sqrt{2}} \epsilon\left(\theta_{0}+\theta_{2} \Phi_{0}\right) & |M|^{1 / 2} \epsilon \theta_{1} & 1
\end{array}\right)
$$

As we can see, the physical Yukawa couplings now depend on the fluxes. Interestingly enough these physical couplings only depend on the dimensionless combinations $\left(\epsilon \theta_{0}|M|\right)$, $\left(\epsilon \theta_{1,2} \Phi_{0}|M|\right)$ and $\left(\epsilon \theta_{1,2}|M|^{1 / 2}\right)$. In other words, there is an invariance under the rescalings

$$
\left(\epsilon \theta_{0}, \epsilon \theta_{1,2}, \Phi_{0}, M\right) \rightarrow\left(\lambda^{2} \epsilon \theta_{0}, \lambda \epsilon \theta_{1,2}, \lambda \Phi_{0}, \lambda^{-2} M\right)
$$

and there is no explicit dependence on the "stringy" scales $m_{*}, m_{\Phi}$. This is as it should since Yukawa couplings in type IIB string compactifications may be computed in the large volume limit just in terms of the compactified 10d field theory, without explicit reference to any string theoretical scale. Note that, had we included $\theta$ corrections to the wave functions in the normalization we would have obtained corrections for each entry of higher order in $\left(\epsilon \theta_{0} M\right),\left(\epsilon \theta_{i} \Phi_{0}|M|\right)$, etc.

\subsection{A model of quark-lepton hierarchies}

We can take the $\mathrm{U}(3) \rightarrow \mathrm{U}(1)$ model as a toy model for the $\mathrm{SO}(12) \rightarrow \mathrm{SU}(5)$ and $E_{6} \rightarrow$ SU(5) symmetry structure underlying the $\mathbf{1 0} \times \overline{\mathbf{5}} \times \overline{\mathbf{5}}_{\mathbf{H}}$ and $\mathbf{1 0} \times \mathbf{1 0} \times \mathbf{5}_{\mathbf{H}}$ Yukawas in a local SU(5) GUT. In F-theory SU(5) GUT's one breaks the gauge symmetry down to the SM by the addition of $F_{Y}$ fluxes along the hypercharge direction. The matter localized at the curves then feel both the flux in the curves and the hypercharge flux in the bulk $S$. Although the net hypercharge flux on the matter curves associated to quark and leptons is assumed to vanish (so that the SM family spectrum is not spoiled), the value of the hypercharge flux density at the intersection Yukawa point is in general non-vanishing. This effect can then make the physical Yukawa couplings sensitive to the hypercharge flux. One can model out this situation by taking the above wave functions with constant fluxes and making the replacement $M=M_{0}+Y M_{1}$ in the physical Yukawa couplings in eq. (6.6). Here $M_{0}, M_{1}$ are the curve flux and the bulk hypercharge flux respectively, and $Y=1,-4,2,-3,6$ are the hypercharges of the SM fields $Q_{L}, U_{R}, D_{R}, L, E_{R}$ respectively, in standard notation. This may be a good approximation to the extent that the local flux in the vicinity of the Yukawa point may be slowly varying. This might be the case recalling that the physical Yukawa coupling and the normalized wave functions are only sensitive to backgrounds in the vicinity of this Yukawa point. Left- and right-handed fermions in a given coupling have different hypercharges $Y_{L, R}$ so that one finds that the ratio of physical Yukawa couplings 
have a hypercharge dependence of the form

$$
\frac{Y}{Y^{33}}=\left(\begin{array}{ccc}
\mathcal{O}\left(\epsilon^{2}\right) & \mathcal{O}\left(\epsilon^{2}\right) & \left(\theta_{0}+\theta_{1} \Phi_{0}\right) \frac{\epsilon}{\sqrt{2}}\left|M_{R}\right| \\
\mathcal{O}\left(\epsilon^{2}\right) & \epsilon\left[\left(\theta_{1}+\theta_{2}\right) \Phi_{0}-\theta_{0}\right]\left|M_{L} M_{R}\right|^{1 / 2} & \epsilon \theta_{2}\left|M_{R}\right|^{1 / 2} \\
\left(\theta_{0}+\theta_{2} \Phi_{0}\right) \frac{\epsilon}{\sqrt{2}}\left|M_{L}\right| & \epsilon \theta_{1}\left|M_{L}\right|^{1 / 2} & 1
\end{array}\right)
$$

where

$$
M_{L}=M_{0}+Y_{L} M_{1}, M_{R}=M_{0}+Y_{R} M_{1} .
$$

Note that the leading QCD low energy running for $D$-quarks cancels out in this matrix, so that it is easier the comparison with the leptonic hierarchies. It is interesting to check numerically whether this kind of structure is able to describe the observed hierarchy of fermion masses and their mixing. Since the above matrix is not hermitian it is simpler to compute the eigenvalues and eigenvectors of its product by its adjoint and take the square root. In SU(5) GUT's the right handed $D$-quarks live in a $\overline{\mathbf{5}}$ matter curve whereas the lefthanded quarks $Q_{L}$ live in a 10. For the leptons the opposite happens, left-handed leptons $L$ live in the $\overline{\mathbf{5}}$ and the right-handed leptons in the 10. This means that when going from a $D$-quark Yukawa matrix to a lepton matrix we have to interchange $Y_{R}, M_{R} \leftrightarrow Y_{L}, M_{L}$. In the case of $U$-quark masses Yukawa couplings come from an intersection $\mathbf{1 0} \times \mathbf{1 0} \times \mathbf{5}_{\mathbf{H}}$, both left and right $U$-quarks are in a $\mathbf{1 0}$ and one has to symmetrize the Yukawa coupling. Note also that in this case, as noted in [8] the 10-plet matter curve must self-pinch or rather both 10-plet branches must be related by some discrete symmetry [14] in order to be able to get eventually rank $=3$ matrices. We will further assume that there is a single intersection point of matter curves for each of the two types of Yukawa couplings.

We have looked for values of the six parameters $\epsilon \theta_{0}, \epsilon \theta_{1}, \epsilon \theta_{2}, \Phi_{0}, M_{0}, M_{1}$ (with all parameters real for simplicity) able to reproduce the observed fermion hierarchies and mixings. Note that in a realistic setting these sets of parameters are different for the $D / L$ and the $U$ physical Yukawa couplings since the corresponding intersection points are in general different in a SU(5) local GUT. We will compare the results with the observed ratios of physical Yukawa couplings all evaluated at a scale of order the electroweak scale. One has for those (see e.g. [53] )

$$
\begin{aligned}
& \left(\frac{Y_{1}}{Y_{3}}\right)_{U}=(0.5-1.6) \cdot 10^{-5} \text { and }\left(\frac{Y_{2}}{Y_{3}}\right)_{U}=(3-4) \cdot 10^{-3} \\
& \left(\frac{Y_{1}}{Y_{3}}\right)_{D}=(0.6-1.8) \cdot 10^{-3} \text { and }\left(\frac{Y_{2}}{Y_{3}}\right)_{D}=(1-3) \cdot 10^{-2} \\
& \left(\frac{Y_{1}}{Y_{3}}\right)_{L}=(2.8) \cdot 10^{-4} \quad \text { and }\left(\frac{Y_{2}}{Y_{3}}\right)_{L}=(5.9) \cdot 10^{-2} .
\end{aligned}
$$

The experimental CKM mixing matrix with $90 \%$ CL is (see [54])

$$
\left|V_{C K M}\right|=\left(\begin{array}{ccc}
0.9741-0.9756 & 0.219-0.226 & 0.0025-0.0048 \\
0.219-0.226 & 0.9732-0.9748 & 0.038-0.044 \\
0.004-0.014 & 0.037-0.044 & 0.9990-0.9993
\end{array}\right)
$$

As an example take parameters

$$
\left(\epsilon \theta_{0}, \epsilon \theta_{1}, \epsilon \theta_{2}, \Phi_{0}, M_{0}, M_{1}\right)_{D, L}=(-0.066,0.10,-0.27,0.5,1.41,-0.31)
$$




$$
\left(\epsilon \theta_{0}, \epsilon \theta_{1}, \epsilon \theta_{2}, \Phi_{0}, M_{0}, M_{1}\right)_{U}=(-0.033,-0.27,-0.33,-0.1,-1.1,-0.47) .
$$

for the backgrounds at the $D / L$ and $U$ Yukawa intersecting points respectively. One then obtains mass ratios

$$
\begin{aligned}
& \left(m_{1}, m_{2}, m_{3}\right)_{U}=\left(6.9 \cdot 10^{-5}, 3.8 \cdot 10^{-3}, 1\right) \\
& \left(m_{1}, m_{2}, m_{3}\right)_{D}=\left(0.65 \cdot 10^{-3}, 2.96 \cdot 10^{-2}, 1\right) \\
& \left(m_{1}, m_{2}, m_{3}\right)_{L}=\left(4.8 \cdot 10^{-4}, 5.3 \cdot 10^{-2}, 1\right)
\end{aligned}
$$

with a CKM mixing matrix

$$
V_{C K M}=\left(\begin{array}{lll}
0.9834 & 0.1812 & 0.0056 \\
0.1809 & 0.9827 & 0.0382 \\
0.0125 & 0.0365 & 0.9992
\end{array}\right)
$$

The agreement is quite good taking into account the simplicity of the model and the uncertainties. In particular the first (smallest) eigenvalues are less reliable since in the computation of the Yukawa couplings we have neglected effects of order $\epsilon^{2}$ which could be relevant for the masses and mixings of the first generation. Many other solutions leading to similarly acceptable results exist. Note that due to the scale invariance in eq. (6.7) the same numerical results may be obtained with magnetic fluxes a factor $1 / \lambda^{2}$ smaller by compensating taking larger values for the rest of the parameters. Note also that in the above we have computed ratios of physical Yukawa couplings. However, flux effects do also affect the relative size of the third generation physical Yukawa couplings. Indeed, from eq. (6.5) one obtains

$$
\frac{Y^{33}(L)}{Y^{33}(D)}=\frac{\left(\mathcal{N}_{D} \mathcal{N}_{Q}\right)^{1 / 2}}{\left(\mathcal{N}_{L} \mathcal{N}_{E}\right)^{1 / 2}}
$$

As we saw, in the dilute flux limit with $m_{*}, m_{\Phi} \gg|M|$ one has $\mathcal{N}_{a} \simeq m_{*}^{4} /\left(m_{\Phi}^{2}|M|\right)$ so

$$
\frac{Y^{33}(L)}{Y^{33}(D)} \simeq \frac{\left(\left|M_{0}+Y_{L} M_{1}\right|\left|M_{0}+Y_{E} M_{1}\right|\right)^{1 / 2}}{\left(\left|M_{0}+Y_{D} M_{1}\right|\left|M_{0}+Y_{Q} M_{1}\right|\right)^{1 / 2}}=\frac{\left(\left|M_{0}-3 M_{1}\right|\left|M_{0}+6 M_{1}\right|\right)^{1 / 2}}{\left(\left|M_{0}+2 M_{1}\right|\left|M_{0}+M_{1}\right|\right)^{1 / 2}} .
$$

For the above choice of parameters this leads to $Y^{33}(L) / Y^{33}(D) \simeq 1.13$, so that the (successful) standard $\mathrm{SU}(5)$ prediction $m_{b}\left(M_{G U T}\right)=m_{\tau}\left(M_{G U T}\right)$ is not much distorted.

In summary, although the above estimations are based on the results obtained for a simple U(3) model with constant magnetic flux, the lesson seems to be more general. Nonperturbative effects from distant 7 -branes sectors (or, equivalently, local closed string $(1,2)$ fluxes) can give rise to the observed hierarchy of quark and lepton masses. On the other hand turning on hypercharge fluxes on SU(5) models seems able to explain the difference in masses between the D-quarks and charged leptons of the lightest generations. Although in a different setup, the effect of hypercharge flux in the leptonic and down type Yukawas was also considered in [13].

\section{Final comments and conclusions}

In this paper we have analyzed the dependence of Yukawa couplings on two important ingredients of F-theory GUT models: 7-brane worldvolume fluxes and corrections which 
are non-perturbative on Kähler moduli couplings. The former are essential in building the local F-theory model itself, since they are needed to obtain a $4 \mathrm{~d}$ chiral spectrum and, in most scenarios, to perform the gauge symmetry breaking $G_{G U T} \rightarrow G_{M S S M}$. The latter can be built independently of the local GUT model, since they take place on extra 7-branes distant from the GUT 4-cycle $S$ (see figure 1), but are nevertheless crucial for achieving full moduli stabilization in the type IIB/F-theory context $[55,56]$.

As shown in [15-17], before such non-perturbative corrections holomorphic Yukawas do not depend on 7-brane worldvolume fluxes, ${ }^{14}$ which implies that the rank of the matrix of physical Yukawas does not depend on them either. Here we have found that the same result applies in the presence of gaugino condensate/D3-instanton effects, and that only such non-perturbative corrections may modify the number of quark/lepton generations that are massive in a local F-theory model.

While the above picture is rather simple, it opens up a quite interesting scenario. First, in the context of the SU(5) models described in the introduction, one needs nonperturbative effects in order to give mass to the two lightest generations of quarks and leptons. The resulting Yukawa matrix then displays a clear hierarchical structure, just like (5.36) for the $\mathrm{U}(3)$ toy model that we have analyzed, but is nevertheless independent of the worldvolume fluxes. As the hypercharge flux is the only ingredient that breaks the GUT symmetry down to the MSSM, the D-quark and lepton Yukawas are identical even after the breaking $G_{G U T} \rightarrow G_{M S S M}$, except from the fact that they have different normalization. Taking into account that wavefunction normalization does depend on worldvolume fluxes, we obtain a matrix of physical Yukawas of the form (6.6). This flux dependence allows to accommodate the observed MSSM mass ratios in a simple toy model of quark/lepton hierarchies.

In fact, the above scenario is quite robust, in the sense that it also applies to F-theory models built in supersymmetric backgrounds with IASD (1,2)-fluxes in the three-fold base, dubbed in the literature as $\beta$-deformed backgrounds. ${ }^{15}$ Indeed, as pointed out in [23], a $\beta$-deformation on the closed string background induces a non-commutative deformation on the GUT 7-brane gauge theory in the sense of [16], and this deformation is equivalent to the one induced by non-perturbative effects on the 7-brane superpotential. We have elaborated on such equivalence and given an specific dictionary between internal wavefunctions in the non-perturbative and non-commutative scenarios, and verified that the Yukawas in both formalisms are identical to first order in the non-perturbative/non-commutative parameter $\epsilon$. It would be interesting to check whether such equivalence holds to higher orders in $\epsilon$.

For the purposes of this paper the non-commutative formalism has been perhaps more useful than the commutative one, in the sense that the flux independence of the Yukawa

\footnotetext{
${ }^{14}$ For the type IIB limit of F-theory, this can be guessed from the fact that worldvolume fluxes enter into the 7-brane action via their density, which depends on the Kähler moduli of the compactification. A dependence of the holomorphic Yukawas on the worldvolume fluxes would then imply a dependence of the superpotential on the Kähler moduli, and the latter is forbidden for type IIB compactifications on Calabi-Yau manifolds from general worldsheet arguments [57].

${ }^{15}$ Actually, such backgrounds have only been built in the type IIB limit. It would be interesting to generalize the concept of $\beta$-deformation to genuine F-theory compactifications.
} 
couplings is explicit. Nevertheless, the commutative formalism is bound to play a more prominent role when generalizing the computations made here for the $\mathrm{U}(3)$ model to more involved F-theory setups. Indeed, as can be deduced from the discussion in section 3, the correction to both the wavefunctions and the Yukawa couplings is not only proportional to the parameter $\epsilon$, but also to the symmetric tensor $d_{a b c}=\operatorname{STr}\left(\mathfrak{t}_{a}, \mathfrak{t}_{b}, \mathfrak{t}_{c}\right)$ of the group of enhanced symmetry $G_{p}$ at the Yukawa point $p$. While for $\mathrm{U}(N)$ Yukawa points $d_{a b c}$ is non-zero, the Yukawa points of main interest in F-theory have either $G_{p}=\mathrm{SO}(2 N)$ or $E_{n}$, for which $d_{a b c}=0$ and hence the non-perturbative corrections to Yukawas would seem to vanish identically.

This is however not so, in the following sense. Recall that in order to arrive to the $\beta$ deformed superpotential (3.39) in the commutative formalism we had to perform the expansion (3.38) on the scalar function $\chi_{0}$ that defines the non-perturbative effect/ $\beta$-deformation. While we based our analysis on the first non-trivial term in the Taylor expansion, we could have considered higher order terms, so that

$$
\frac{1}{2} \int_{S} \mathrm{~S} \operatorname{Tr}\left(\chi_{0} F \wedge F\right)=\left.\chi_{0}\right|_{S} N_{D 3}+\frac{1}{2} \int_{S} \theta \operatorname{Tr}\left(\Phi_{x y} F \wedge F\right)+\frac{1}{4} \int_{S} \theta^{\prime} \mathrm{S} \operatorname{Tr}\left(\Phi_{x y}^{2} F \wedge F\right)+\ldots
$$

where again $\left.\theta \equiv m_{\Phi}^{-2} \partial_{z} \chi_{0}\right|_{S}$ and we have defined $\left.\theta^{\prime} \equiv m_{\Phi}^{-4} \partial_{z}^{2} \chi_{0}\right|_{S}$. The second term in this expansion vanishes identically whenever $d_{a b c}=0$, but the third one does not. Hence for those cases where, e.g., $G_{p}=\mathrm{SO}(2 N)$ we will have the total superpotential

$$
W_{\text {total }}=m_{*}^{4}\left[\int_{S} \operatorname{Tr}\left(\Phi_{x y} F\right) \wedge d x \wedge d y+\frac{\epsilon}{4} \int_{S} \theta^{\prime} \mathrm{S} \operatorname{Tr}\left(\Phi_{x y}^{2} F \wedge F\right)\right]
$$

instead of the previous expression (3.39). Naively, for the purpose of computing Yukawa couplings, this new superpotential can be related to the previous one by the replacement $\theta \rightarrow \theta^{\prime}\left\langle\Phi_{x y}\right\rangle$, so one would expect that the results derived for $\mathrm{U}(N)$ groups via (3.39) have an analogue for $\mathrm{SO}(2 N)$ groups in terms of this new superpotential. It is however of obvious interest to generalize the analysis of this paper to include the above superpotential. In particular, it would be interesting to see whether it can also be understood as a non-commutative deformation of (2.1). These and other related issues are currently under investigation.

\section{Acknowledgments}

We thank P.G. Cámara, I. García-Etxebarria, L. Martucci, S. Theisen and A. Uranga for useful discussions. F.M. thanks the CERN TH group and HKUST-IAS for hospitality during the completion of this paper. A.F. thanks the IFT UAM/CSIC, the AEI-Potsdam, as well as the AS ICTP, for hospitality and support during progress of this work; and CDCH-UCV for a research grant No. PI-03-007127-2008. This work has been partially supported by the grants FPA 2009-09017, FPA 2009-07908, Consolider-CPAN (CSD200700042) from the MICINN, HEPHACOS-S2009/ESP1473 from the C.A. de Madrid and the contract "UNILHC" PITN-GA-2009-237920 of the European Commission. F.M. is supported by the MICINN Ramón y Cajal programme through the grant RYC-2009-05096. 


\section{A Magnetized D9-branes and KK modes}

The aim of this section is to describe the intersecting 7-brane models discussed in the text from the T-dual vantage point of magnetized D9-branes, which have been extensively studied for the purpose of computing Yukawa couplings [4, 38, 58-60]. As we will see this D9-brane picture will also allow to describe the spectrum of open string massive modes in a rather natural way. Recall from section 2 that the local intersecting 7-brane configuration considered in the main text are based on a compact divisor $S$ of a threefold $B$, and that Yukawas arise from points of intersection of matter curves $\Sigma_{i} \subset S$. Near such intersection points we can describe our geometry by the local holomorphic coordinates $(x, y, z) \in B$, with $(x, y) \in S, z$ a transverse coordinate to $S$, and such that the fundamental form of $S$ is given by (2.5).

To proceed, let us assume that all the 7-branes are in fact D7-branes and that $B$ is elliptically fibered over $S$. We can then perform fiberwise T-duality to a system of magnetized D9-branes on a threefold $\tilde{B}$. While the D7-brane worldvolume fluxes $F_{x \bar{x}}$, etc. remain as magnetic fluxes in the D9-brane picture, the D7-brane field $\Phi$ is translated into the D9-brane magnetic fluxes $F_{x \bar{z}}=D_{x} \Phi_{x y}$ and $F_{y \bar{z}}=D_{y} \Phi_{x y}$. Hence, a generic system of intersecting D7-branes, for which $\left\langle\Phi_{x y}\right\rangle \neq 0$, translates into a D9-brane system where $F_{x \bar{z}}$ and $F_{y \bar{z}}$ have a non-trivial background value.

For concreteness, let us consider the U(3) model introduced in subsection 2.3. ${ }^{16}$ After performing two T-dualities along the transverse coordinates $(z, \bar{z})$, one obtains a model with a stack of 3 D9-branes, with a gauge flux $F_{D 9}$ given by

$$
\begin{aligned}
\left\langle F_{D 9}\right\rangle= & i\left(M_{x} d x \wedge d \bar{x}+M_{y} d y \wedge d \bar{y}\right) \frac{1}{3}\left(\begin{array}{ccc}
1 & & \\
& -2 & \\
& & 1
\end{array}\right) \\
& +\frac{M_{\Phi_{x}}}{3}\left(\begin{array}{ccc}
-2 & & \\
& 1 & \\
& & 1
\end{array}\right) d \bar{x} \wedge d z+\frac{M_{\Phi_{y}}}{3}\left(\begin{array}{lll}
1 & \\
& 1 & \\
& & -2
\end{array}\right) d \bar{y} \wedge d z+\text { c.c. }
\end{aligned}
$$

where the first line arises from T-dualizing (2.17), while the second line is the T-dual version of (2.12) for $M_{\Phi_{x}}=m_{\Phi_{x}}^{2}$ and $M_{\Phi_{y}}=m_{\Phi_{y}}^{2}$. In addition, the piece of (2.12) proportional to $\Phi_{0}$ will translate into a Wilson line background for $A_{\bar{z}}$, which for simplicity is set to zero in the following.

Similarly to the D7-brane picture, the D9-brane background (A.1) breaks the gauge group as $\mathrm{U}(3) \rightarrow \mathrm{U}(1)^{3}$. The wavefunctions for open string zero modes can then be computed by simply solving the Dirac or Laplace equations. One may do so by choosing a

\footnotetext{
${ }^{16}$ In particular, one may easily embed this U(3) model into a global geometry such as $\left(\mathbf{T}^{2}\right)_{x} \times\left(\mathbf{T}^{2}\right)_{y} \times\left(\mathbf{T}^{2}\right)_{z}$ or orbifolds thereof, so that $S=\left(\mathbf{T}^{2}\right)_{x} \times\left(\mathbf{T}^{2}\right)_{y}$ and to arrive to the magnetized D9-brane picture we simply need to perform two T-dualities along $\left(\mathbf{T}^{2}\right)_{z}$.
} 
gauge for $A$ analogous to (2.28), which in the D9-brane picture reads

$$
\begin{aligned}
\left\langle A_{D 9}\right\rangle= & -\frac{i}{3}\left(M_{x} \bar{x} d x+M_{y} \bar{y} d y\right)\left(\begin{array}{ccc}
1 & & \\
& -2 & \\
& & 1
\end{array}\right) \\
& +\frac{2}{3} M_{\Phi_{x}} \operatorname{Re}(\bar{x} d z)\left(\begin{array}{ccc}
-2 & & \\
& 1 & \\
& & 1
\end{array}\right)+\frac{2}{3} M_{\Phi_{y}} \operatorname{Re}(\bar{y} d z)\left(\begin{array}{lll}
1 & & \\
& 1 & \\
& & -2
\end{array}\right)
\end{aligned}
$$

Unlike in the D7-brane case, (A.2) is not a pure holomorphic gauge because $\left\langle A_{\bar{z}}\right\rangle \neq 0$. Note, however, that a purely holomorphic gauge would imply a $z$-dependent $\left\langle A_{D 9}\right\rangle$, which would prevent to T-dualize this background back to a system of intersecting D7-branes.

Given (A.2), and a local set of coordinates such that the fundamental form of $\tilde{B}$ reads

$$
\mathcal{J}=\frac{i}{2}(d x \wedge d \bar{x}+d y \wedge d \bar{y}+d z \wedge d \bar{z}) .
$$

it is straightforward to write down the zero mode equation for the D9-brane fermionic degrees of freedom (see e.g. $[4,39,40]$ ). Indeed, we may describe them by a negative chirality 10d Majorana-Weyl spinor $\chi$ in the adjoint of $\mathrm{U}(3)$, which we decompose as

$$
\chi=\zeta+\mathcal{B}^{*} \zeta^{*} \quad \zeta=\chi_{4} \otimes \chi_{6}
$$

with $\chi_{6}$ a $6 \mathrm{~d}$ Weyl spinor of negative chirality, $\mathcal{B}=\mathcal{B}_{4} \otimes \mathcal{B}_{6}$ a Majorana matrix and $\chi_{4}$ is a $4 \mathrm{~d}$ Weyl spinor of positive chirality satisfying

$$
\gamma_{(4)} \not_{\mathbb{R}^{1,3}} \mathcal{B}_{4}^{*} \chi_{4}^{*}=-m_{\rho} \chi_{4}
$$

where $\gamma_{(4)}$ is the chirality operator in $4 \mathrm{~d}$ (see subsection A.2 for explicit expressions). Then, the $10 \mathrm{~d}$ Dirac equation $\not D_{10} \chi=0$ reduces to the $6 \mathrm{~d}$ internal KK mode equation

$$
\not D_{\tilde{B}} \chi_{6}=m_{\rho} \mathcal{B}_{6}^{*} \chi_{6}^{*}
$$

with $\not D_{\tilde{B}}=\tilde{\gamma}^{m} D_{m}$ the Dirac operator on the internal 6 d manifold $\tilde{B}$. Taking the conventions of subsection A.2, this last equation can be expressed as

$$
i \mathbf{D}_{\mathbf{A}} \Psi=m_{\rho} \Psi^{\dagger}
$$

with

$$
\Psi=\left(\begin{array}{c}
\psi^{0} \\
\psi^{1} \\
\psi^{2} \\
\psi^{3}
\end{array}\right) \quad \Psi^{\dagger}=\left(\begin{array}{c}
\left(\psi^{0}\right)^{\dagger} \\
\left(\psi^{1}\right)^{\dagger} \\
\left(\psi^{2}\right)^{\dagger} \\
\left(\psi^{3}\right)^{\dagger}
\end{array}\right)
$$

and

$$
\mathbf{D}_{\mathbf{A}}=\left(\begin{array}{cccc}
0 & D_{x} & D_{y} & D_{z} \\
-D_{x} & 0 & -D_{\bar{z}} & D_{\bar{y}} \\
-D_{y} & D_{\bar{z}} & 0 & -D_{\bar{x}} \\
-D_{z} & -D_{\bar{y}} & D_{\bar{x}} & 0
\end{array}\right)
$$


with $D_{m}=\partial_{m}-i\left[A_{m}, \cdot\right]$ the usual covariant derivative. Note that in principle (A.7) for $m_{\rho}=0$ is not the same zero mode equation as (2.9), since in (A.9) $D_{\bar{z}}=\partial_{\bar{z}}-i\left[A_{\bar{z}}, \cdot\right]$. However, if we want to T-dualize along the $(z, \bar{z})$ coordinates back to a system of intersecting D7-branes, we need to smooth out our wavefunctions $\psi^{m}$ along such coordinates, and more precisely we need to impose that $\partial_{z} \Psi=\partial_{\bar{z}} \Psi=0$. Hence, under such assumption the operators $D_{z}, D_{\bar{z}}$ in (A.9) reduce to those in (2.10), and so solutions to (A.7) are also solutions to (2.9) and (2.8).

Finally, applying twice (A.7) we obtain

$$
\mathbf{D}_{\mathbf{A}}^{\dagger} \mathbf{D}_{\mathbf{A}} \Psi=\left|m_{\rho}\right|^{2} \Psi
$$

where

$$
\mathbf{D}_{\mathbf{A}}^{\dagger}=\left(\begin{array}{cccc}
0 & D_{\bar{x}} & D_{\bar{y}} & D_{\bar{z}} \\
-D_{\bar{x}} & 0 & -D_{z} & D_{y} \\
-D_{\bar{y}} & D_{z} & 0 & -D_{x} \\
-D_{\bar{z}} & -D_{y} & D_{x} & 0
\end{array}\right)
$$

and so by solving such eigenvalue equation we can also obtain the wavefunctions for the D9-brane massive replicas of the previous zero modes. Again, in order to translate such result to a D7-brane wavefunction we need to assume that $\Psi$ is $(z, \bar{z})$-independent.

As in the main text, in order to solve for the most general eigenmode of (A.10) it is useful to notice that

$$
\mathbf{D}_{\mathbf{A}}{ }^{\dagger} \mathbf{D}_{\mathbf{A}}=-\Delta \mathbb{I}_{4}-i \mathbf{F} \quad \mathbf{F}=\left(\begin{array}{cccc}
\sigma_{+++} & F_{\bar{y} \bar{z}} & F_{\bar{z} \bar{x}} & F_{\bar{x} \bar{y}} \\
F_{z y} & \sigma_{+--} & F_{y \bar{x}} & F_{z \bar{x}} \\
F_{x z} & F_{x \bar{y}} & \sigma_{-+-} & F_{z \bar{y}} \\
F_{y x} & F_{x \bar{z}} & F_{y \bar{z}} & \sigma_{--+}
\end{array}\right)
$$

where $\Delta$ and $\sigma_{\epsilon_{x} \epsilon_{y} \epsilon_{z}}$ are defined as in (2.19), (2.20), respectively. If the entries of $\mathbf{F}$ are constant, then the operators $\Delta \mathbb{I}_{4}$ and $\mathbf{F}$ commute and one can diagonalize them simultaneously. Indeed, imposing the F-term condition $F^{(0,2)}=0$, one finds that the solutions for (A.10) are of the form ${ }^{17}$

$$
\begin{aligned}
& \left|m_{\rho}\right|^{2}=\rho^{2}-i \sigma_{+++}, \Psi=\left(\begin{array}{l}
1 \\
0 \\
0 \\
0
\end{array}\right) \psi_{\rho} \quad\left|m_{\rho}\right|^{2}=\rho^{2}+\lambda_{1}, \Psi=J\left(\begin{array}{l}
0 \\
1 \\
0 \\
0
\end{array}\right) \psi_{\rho} \\
& \left|m_{\rho}\right|^{2}=\rho^{2}+\lambda_{2}, \Psi=J\left(\begin{array}{l}
0 \\
0 \\
1 \\
0
\end{array}\right) \psi_{\rho} \quad\left|m_{\rho}\right|^{2}=\rho^{2}+\lambda_{3}, \Psi=J\left(\begin{array}{l}
0 \\
0 \\
0 \\
1
\end{array}\right) \psi_{\rho}
\end{aligned}
$$

\footnotetext{
${ }^{17}$ Imposing the D9-brane D-term condition $\operatorname{Tr} F^{2} \wedge J=0$ implies that $\sigma_{+++}=0$ and so $\lambda_{1}+\lambda_{2}+\lambda_{3}=0$.
} 
where $-\Delta \psi_{\rho}=\rho^{2} \psi_{\rho}$, and the $4 \times 4$ unitary matrix $J$ is defined by

$$
J=\left(\begin{array}{ll}
1 & 0 \\
0 & j
\end{array}\right) \quad \text { such that } \quad J^{-1} \mathbf{F} J=i\left(\begin{array}{lll}
-i \sigma_{+++} & & \\
& \lambda_{1} & \\
& & \\
& \lambda_{2} & \\
& & \lambda_{3}
\end{array}\right)
$$

In particular, the fermionic zero mode satisfying the Dirac equation (A.7) for $m_{\rho}=0$ will be of this form. Let us for instance assume that it has of the form of the second solution in (A.13b). Then it is easy to see that the corresponding Laplace eigenfunction $\psi_{0}$ is such that $\rho^{2}=-\lambda_{3}$ and

$$
\mathcal{D}_{\bar{x}} \psi_{0}=\mathcal{D}_{\bar{y}} \psi_{0}=\mathcal{D}_{z} \psi_{0}=0
$$

where the operators $\mathcal{D}_{m}$ are linear combinations of the covariant derivatives $D_{m}$ defined by

$$
\left(\begin{array}{l}
\mathcal{D}_{x} \\
\mathcal{D}_{y} \\
\mathcal{D}_{z}
\end{array}\right)=j^{t}\left(\begin{array}{c}
D_{x} \\
D_{y} \\
D_{z}
\end{array}\right) \quad \text { and } \quad\left(\begin{array}{c}
\mathcal{D}_{\bar{x}} \\
\mathcal{D}_{\bar{y}} \\
\mathcal{D}_{\bar{z}}
\end{array}\right)=j^{-1}\left(\begin{array}{c}
D_{\bar{x}} \\
D_{\bar{y}} \\
D_{\bar{z}}
\end{array}\right)
$$

To obtain all possible eigenvalues of $\Delta$ we can use its commutation relations with the $\mathcal{D}_{m}$ to show that $\mathcal{D}_{x}, \mathcal{D}_{y}$ and $\mathcal{D}_{\bar{z}}$ act as creation operators whereas $\mathcal{D}_{\bar{x}}, \mathcal{D}_{\bar{y}}$ and $\mathcal{D}_{z}$ are annihilation operators as implied by (A.15). The final result is that the eigenfunctions of $\Delta$ are all of the form

$$
\psi^{m n l}=\left(\mathcal{D}_{x}\right)^{m}\left(\mathcal{D}_{y}\right)^{n}\left(\mathcal{D}_{\bar{z}}\right)^{l} \psi_{0}
$$

Note that in the above discussion we have suppressed the gauge indices of $\psi_{\rho}$, which in practice will be a matrix of the form (2.16) with only one non-vanishing entry or sector $\alpha^{ \pm}$. As the worldvolume flux $F_{n \bar{m}} \sim\left[F_{n \bar{m}}, \cdot\right]$ acts in the adjoint for such gauge indices, each sector (i.e., each matter curves in the T-dual D7-brane picture) will feel a different Laplace operator $\Delta$, a different matrix $\mathbf{F}$ and a different rotation matrix $J$. Rather than giving a description of these sectors for a general D9-brane model, we will illustrate them for the particular case of the $\mathrm{U}(3)$ model introduced in subsection 2.3.

\section{A.1 Massive modes for the U(3) model}

\section{Sector $a$}

Similarly to section 2 , the matrix $\mathbf{F}$ for the sectors $a^{ \pm}$reads

$$
\mathbf{F}_{a^{ \pm}}= \pm i\left(\begin{array}{cccc}
\frac{1}{2}\left(M_{x}+M_{y}\right) & 0 & 0 & 0 \\
0 & \frac{1}{2}\left(M_{x}-M_{y}\right) & 0 & -i M_{\Phi_{x}} \\
0 & 0 & -\frac{1}{2}\left(M_{x}-M_{y}\right) & 0 \\
0 & i M_{\Phi_{x}} & 0 & -\frac{1}{2}\left(M_{x}+M_{y}\right)
\end{array}\right)
$$

Note that in order to reproduce eq. (2.21) we just need to impose $M_{\Phi_{x}}=m_{\Phi}^{2}$. The rotation matrix that satisfies (A.14) is given by

$$
J_{a}=\left(\begin{array}{cc}
1 & 0 \\
0 & j_{a}
\end{array}\right), \quad \text { where } \quad j_{a}=\left(\begin{array}{ccc}
\cos \phi_{a} & 0 & -i \sin \phi_{a} \\
0 & 1 & 0 \\
-i \sin \phi_{a} & 0 & \cos \phi_{a}
\end{array}\right)
$$


where we have defined

$$
\cos \phi_{a}=\frac{1}{\sqrt{\left(\tilde{\lambda}_{a,-}\right)^{2}+1}} \quad \sin \phi_{a}=\frac{\tilde{\lambda}_{a,-}}{\sqrt{\left(\tilde{\lambda}_{a,-}\right)^{2}+1}}
$$

and

$$
\lambda_{a, \pm}=\frac{M_{x}}{2} \pm \sqrt{\left(\frac{M_{x}}{2}\right)^{2}+M_{\Phi_{x}}^{2}} \quad \tilde{\lambda}_{a, \pm}=\frac{\lambda_{a, \pm}}{M_{\Phi_{x}}}
$$

Indeed, it is easy to check that

$$
\mathcal{D}_{\mathbf{A}}=J_{a}^{t} \cdot \mathbf{D}_{\mathbf{A}} \cdot J_{a}=\left(\begin{array}{cccc}
0 & \mathcal{D}_{x} & \mathcal{D}_{y} & \mathcal{D}_{z} \\
-\mathcal{D}_{x} & 0 & -\mathcal{D}_{\bar{z}} & \mathcal{D}_{\bar{y}} \\
-\mathcal{D}_{y} & \mathcal{D}_{\bar{z}} & 0 & -\mathcal{D}_{\bar{x}} \\
-\mathcal{D}_{z} & -\mathcal{D}_{\bar{y}} & \mathcal{D}_{\bar{x}} & 0
\end{array}\right)
$$

where, as in (A.16), we have

$$
\begin{aligned}
& \left(\begin{array}{l}
\mathcal{D}_{x} \\
\mathcal{D}_{y} \\
\mathcal{D}_{z}
\end{array}\right)=j_{a}^{t}\left(\begin{array}{c}
D_{x} \\
D_{y} \\
D_{z}
\end{array}\right)=\left(\begin{array}{c}
c_{a} D_{x}-i s_{a} D_{z} \\
D_{y} \\
c_{a} D_{z}-i s_{a} D_{x}
\end{array}\right)=\left(\begin{array}{c}
c_{a}\left(\partial_{x} \mp \bar{x} \lambda_{a,+}\right) \\
\partial_{y} \mp \bar{y} M_{y} \\
-i s_{a}\left(\partial_{x} \mp \bar{x} \lambda_{a,-}\right)
\end{array}\right) \\
& \left(\begin{array}{c}
\mathcal{D}_{\bar{x}} \\
\mathcal{D}_{\bar{y}} \\
\mathcal{D}_{\bar{z}}
\end{array}\right)=j_{a}^{-1}\left(\begin{array}{c}
D_{\bar{x}} \\
D_{\bar{y}} \\
D_{\bar{z}}
\end{array}\right)=\left(\begin{array}{c}
c_{a} D_{\bar{x}}+i s_{a} D_{\bar{z}} \\
D_{\bar{y}} \\
c_{a} D_{\bar{z}}+i s_{a} D_{\bar{x}}
\end{array}\right)=\left(\begin{array}{c}
c_{a}\left(\partial_{\bar{x}} \mp x \lambda_{a,-}\right) \\
\partial_{\bar{y}} \\
i s_{a}\left(\partial_{\bar{x}} \mp x \lambda_{a,+}\right)
\end{array}\right)
\end{aligned}
$$

in the semi-holomorphic gauge (A.2) and for the $a^{ \pm}$sectors respectively, and where we have taken the abbreviations $c_{a}=\cos \phi_{a}, s_{a}=\sin \phi_{a}$. One may also check that in this rotated basis the operator (A.12) becomes

$$
\mathcal{D}_{\mathbf{A}}^{\dagger} \mathcal{D}_{\mathbf{A}}=J_{a}^{-1} \mathbf{D}_{\mathbf{A}}^{\dagger} \mathbf{D}_{\mathbf{A}} J_{a}=-\left(\Delta_{a^{ \pm}} \pm M_{x y}\right) \mathbb{I}_{4} \pm\left(\begin{array}{cccc}
2 M_{x y} & 0 & 0 & 0 \\
0 & \lambda_{a,+} & 0 & 0 \\
0 & 0 & M_{y} & 0 \\
0 & 0 & 0 & \lambda_{a,-}
\end{array}\right)
$$

where $M_{x y} \equiv \frac{1}{2}\left(M_{x}+M_{y}\right)$ and $\Delta$ may be expressed either by (2.19) or by

$$
\Delta=\left\{\mathcal{D}_{x}, \mathcal{D}_{\bar{x}}\right\}+\left\{\mathcal{D}_{y}, \mathcal{D}_{\bar{y}}\right\}+\left\{\mathcal{D}_{z}, \mathcal{D}_{\bar{z}}\right\}
$$

From (A.24) it is easy to see that the eigenvectors of $\mathbf{D}_{\mathbf{A}}{ }^{\dagger} \mathbf{D}_{\mathbf{A}}$ in the $a^{ \pm}$sector are indeed of the form (A.13), with the replacement $J \rightarrow J_{a}$. For $M_{x}<0<M_{y}$, the lowest eigenvalue in the $a^{-}$sector will arise from the first eigenvector in (A.13b) with lowest eigenvalue of $\Delta_{a^{-}}$, while in the $a^{+}$sector it will arise from the second eigenvector in (A.13b) with lowest eigenvalue of $\Delta_{a^{+}}$. Similarly to the discussion around eq. (2.27) one can see that only the sector $a^{+}$contains zero modes, which in the semi-holomorphic gauge (A.2) are given by

$$
\Psi_{0, a^{+}}=J_{a}\left(\begin{array}{l}
0 \\
0 \\
0 \\
1
\end{array}\right) \psi_{0, a^{+}}, \quad \psi_{0, a^{+}}=e^{\lambda_{a,-}|x|^{2}} f_{a}(y)
$$


with $f_{a}$ a holomorphic function to be determined by boundary conditions. Since the wavefunction $\psi_{0, a^{+}}$satisfies

$$
\mathcal{D}_{\bar{x}} \psi_{0, a^{+}}=\mathcal{D}_{\bar{y}} \psi_{0, a^{+}}=\mathcal{D}_{z} \psi_{0, a^{+}}=0
$$

for the operators $\mathcal{D}_{m}$ of the $a^{+}$sector, it is easy to check that (A.26) satisfies the zero mode equation (2.9). From (A.27) and the commutation relations $\left[\mathcal{D}_{m}, \mathcal{D}_{\bar{m}}\right]$ one can also check that $\psi_{0, a^{+}}$is an eigenfunction of the Laplacian such that

$$
\Delta_{a^{+}} \psi_{0, a^{+}}=-\frac{1}{2}\left(\left(\lambda_{a,+}-\lambda_{a,-}\right)+M_{y}\right) \psi_{0, a^{+}}=\left(\lambda_{a,-}-M_{x y}\right) \psi_{0, a^{+}}
$$

as expected from the fact that (A.26) is a zero mode of $\mathbf{D}_{\mathbf{A}}^{\dagger} \mathbf{D}_{\mathbf{A}}$ as well. In fact, the commutation relations $\left[\mathcal{D}_{m}, \mathcal{D}_{\bar{m}}\right]$ allow to reproduce the whole spectrum of eigenvalues of $\Delta_{a^{+}}$. Indeed, let us first rewrite them as

$$
\begin{aligned}
{\left[\Delta_{a^{+}}, \mathcal{D}_{x}\right] } & =-\lambda_{a,+} \mathcal{D}_{x} & {\left[\Delta_{a^{+}}, \mathcal{D}_{\bar{x}}\right] } & =\lambda_{a,+} \mathcal{D}_{\bar{x}} \\
{\left[\Delta_{a^{+}}, \mathcal{D}_{y}\right] } & =-M_{y} \mathcal{D}_{y} & {\left[\Delta_{a^{+}}, \mathcal{D}_{\bar{y}}\right] } & =M_{y} \mathcal{D}_{\bar{y}} \\
{\left[\Delta_{a^{+}}, \mathcal{D}_{z}\right] } & =-\lambda_{a,-} \mathcal{D}_{z} & {\left[\Delta_{a^{+}}, \mathcal{D}_{\bar{z}}\right] } & =\lambda_{a,-} \mathcal{D}_{\bar{z}}
\end{aligned}
$$

from which it is easy to see that the remaining eigenfunctions are of the form

$$
\psi_{m n l, a^{+}}=\left(\mathcal{D}_{x}\right)^{m}\left(\mathcal{D}_{y}\right)^{n}\left(\mathcal{D}_{\bar{z}}\right)^{l} \psi_{0, a^{+}}
$$

with eigenvalue $-\left(m \lambda_{a,+}+n M_{y}-(l+1) \lambda_{a,-}+M_{x y}\right)$. The full set of eigenfunctions and eigenvalues of $\mathbf{D}_{\mathbf{A}}{ }^{\dagger} \mathbf{D}_{\mathbf{A}}$ for the $a^{+}$sector is displayed in table 1.

\section{Sector $b$}

For sectors $b^{ \pm}$we have that the matrix $\mathbf{F}$ reads

$$
\mathbf{F}_{b^{ \pm}}= \pm i\left(\begin{array}{cccc}
-M_{x y} & 0 & 0 & 0 \\
0 & -M_{x}+M_{x y} & 0 & 0 \\
0 & 0 & -M_{y}+M_{x y} & i M_{\Phi_{y}} \\
0 & 0 & -i M_{\Phi_{y}} & M_{x y}
\end{array}\right)
$$

and so

$$
J_{b}=\left(\begin{array}{cc}
1 & 0 \\
0 & j_{b}
\end{array}\right), \quad \text { where } \quad j_{b}=\left(\begin{array}{ccc}
1 & 0 & 0 \\
0 & \cos \phi_{b} & i \sin \phi_{b} \\
0 & i \sin \phi_{b} & \cos \phi_{b}
\end{array}\right)
$$

where now the rotation angle is specified by

$$
c_{b}=\cos \phi_{b}=\frac{1}{\sqrt{\left(\tilde{\lambda}_{b,-}\right)^{2}+1}} \quad s_{b}=\sin \phi_{b}=\frac{\tilde{\lambda}_{b,-}}{\sqrt{\left(\tilde{\lambda}_{b,-}\right)^{2}+1}}
$$

and

$$
\lambda_{b, \pm}=-\frac{M_{y}}{2} \pm \sqrt{\left(\frac{M_{y}}{2}\right)^{2}+M_{\Phi_{y}}^{2}} \quad \tilde{\lambda}_{b, \pm}=\frac{\lambda_{b, \pm}}{M_{\Phi_{y}}}
$$




\begin{tabular}{|c|c|}
\hline mass $^{2}$ & $a^{+}$mode $H_{a^{+}}$ \\
\hline$m \lambda_{a,+}+n M_{y}+l\left|\lambda_{a,-}\right|$ & $\left(\begin{array}{c}0 \\
-\frac{i \lambda_{a,-}}{M_{\Phi_{x}}} \\
0 \\
1\end{array}\right) \psi_{m n l}$ \\
\hline$m \lambda_{a,+}+(n+1) M_{y}+(l+1)\left|\lambda_{a,-}\right|$ & $\left(\begin{array}{c}0 \\
0 \\
1 \\
0\end{array}\right) \psi_{m n l}$ \\
\hline$(m+1) \lambda_{a,+}+n M_{y}+(l+1)\left|\lambda_{a,-}\right|$ & $\left(\begin{array}{c}0 \\
1 \\
0 \\
i \lambda_{a,-} \\
M_{\Phi_{x}}\end{array}\right) \psi_{m n l}$ \\
\hline$m \lambda_{a,+}+n M_{y}+(l+1)\left|\lambda_{a,-}\right|+2 M_{x y}$ & $\left(\begin{array}{l}1 \\
0 \\
0 \\
0\end{array}\right) \psi_{m n l}$ \\
\hline
\end{tabular}

Table 1. Massive modes in the $a^{+}$sector. Here $\psi_{m n l}=\psi_{m n l, a^{+}}$is constructed as in (A.30).

matching the rotation angle in the $a^{ \pm}$sectors for $M_{x}=-M_{y}$ and $M_{\Phi_{y}}=m_{\Phi}^{2}$. Again, we can define a rotated Dirac operator $\mathcal{D}_{A}$ as in (A.22), with the replacement $J_{a} \rightarrow J_{b}$. The entries of such operator are then given by

$$
\begin{aligned}
& \left(\begin{array}{l}
\mathcal{D}_{x} \\
\mathcal{D}_{y} \\
\mathcal{D}_{z}
\end{array}\right)=j_{b}^{t}\left(\begin{array}{c}
D_{x} \\
D_{y} \\
D_{z}
\end{array}\right)=\left(\begin{array}{c}
D_{x} \\
c_{b} D_{y}+i s_{b} D_{z} \\
c_{b} D_{z}+i s_{b} D_{x}
\end{array}\right)=\left(\begin{array}{c}
\partial_{x} \pm \bar{x} M_{x} \\
c_{b}\left(\partial_{y} \mp \bar{y} \lambda_{b,+}\right) \\
i s_{b}\left(\partial_{y} \mp \bar{y} \lambda_{b,-}\right)
\end{array}\right) \\
& \left(\begin{array}{c}
\mathcal{D}_{\bar{x}} \\
\mathcal{D}_{\bar{y}} \\
\mathcal{D}_{\bar{z}}
\end{array}\right)=j_{b}^{-1}\left(\begin{array}{c}
D_{\bar{x}} \\
D_{\bar{y}} \\
D_{\bar{z}}
\end{array}\right)=\left(\begin{array}{c}
D_{\bar{x}} \\
c_{b} D_{\bar{y}}-i s_{a} D_{\bar{z}} \\
c D_{\bar{z}}-i s D_{\bar{y}}
\end{array}\right)=\left(\begin{array}{c}
\partial_{\bar{x}}\left(\partial_{\bar{y}} \mp y \lambda_{b,-}\right) \\
i s_{b}\left(\partial_{\bar{y}} \mp y \lambda_{b,+}\right)
\end{array}\right)
\end{aligned}
$$

in the semi-holomorphic gauge (A.2) and for the $b^{ \pm}$sectors respectively. In this rotated basis we have

$$
\mathcal{D}_{\mathbf{A}}^{\dagger} \mathcal{D}_{\mathbf{A}}=J_{b}^{-1} \mathbf{D}_{\mathbf{A}}^{\dagger} \mathbf{D}_{\mathbf{A}} J_{b}=-\left(\Delta_{b^{ \pm}} \mp M_{x y}\right) \mathbb{I}_{4} \pm\left(\begin{array}{cccc}
-2 M_{x y} & 0 & 0 & 0 \\
0 & -M_{x} & 0 & 0 \\
0 & 0 & \lambda_{b,+} & 0 \\
0 & 0 & 0 & \lambda_{b,-}
\end{array}\right)
$$

and so the eigenvalues of $\mathbf{D}_{\mathbf{A}}^{\dagger} \mathbf{D}_{\mathbf{A}}$ in the $b^{ \pm}$sector are also of the form (A.13), now with the replacement $J \rightarrow J_{b}$. For $M_{x}<0<M_{y}$, the zero mode arises in the $b^{+}$sector, and it 
is given by

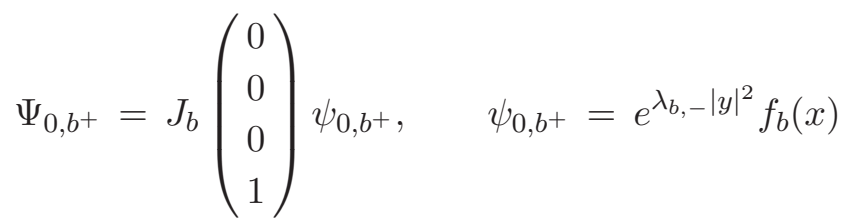

with $f_{b}$ holomorphic. Note that again, in the $b^{+}$sector

$$
\mathcal{D}_{\bar{x}} \psi_{0, b^{+}}=\mathcal{D}_{\bar{y}} \psi_{0, b^{+}}=\mathcal{D}_{z} \psi_{0, b^{+}}=0
$$

and so the spectrum of eigenvectors can be obtained by using an algebra of creation an annihilation operators. Such algebra is codified in the commutation relations

$$
\begin{aligned}
{\left[\Delta_{b^{ \pm}}, \mathcal{D}_{x}\right] } & = \pm M_{x} \mathcal{D}_{x} & {\left[\Delta_{b^{ \pm}}, \mathcal{D}_{\bar{x}}\right] } & =\mp M_{x} \mathcal{D}_{\bar{x}} \\
{\left[\Delta_{b^{ \pm}}, \mathcal{D}_{y}\right] } & =\mp \lambda_{b,+} \mathcal{D}_{y} & {\left[\Delta_{b^{ \pm}}, \mathcal{D}_{\bar{y}}\right] } & = \pm \lambda_{b,+} \mathcal{D}_{\bar{y}} \\
{\left[\Delta_{b^{ \pm}}, \mathcal{D}_{z}\right] } & =\mp \lambda_{b,-} \mathcal{D}_{z} & {\left[\Delta_{b^{ \pm}}, \mathcal{D}_{\bar{z}}\right] } & = \pm \lambda_{b,-} \mathcal{D}_{\bar{z}}
\end{aligned}
$$

that show that the wavefunction

$$
\psi_{m n l, b^{+}}=\left(\mathcal{D}_{x}\right)^{m}\left(\mathcal{D}_{y}\right)^{n}\left(\mathcal{D}_{\bar{z}}\right)^{l} \psi_{0, b^{+}}
$$

is an eigenfunction of $\Delta_{b^{+}}$with eigenvalue $\left(m M_{x}-n \lambda_{b,+}+(l+1) \lambda_{b,-}+M_{x y}\right)$. From this we can construct the massive modes in the $b^{+}$sector, following the same steps as in the sector $a^{+}$.

\section{Sector $c$}

For this sector we have

$$
\mathbf{F}_{c^{ \pm}}= \pm i\left(\begin{array}{cccc}
0 & 0 & 0 & 0 \\
0 & 0 & 0 & i M_{\Phi_{x}} \\
0 & 0 & 0 & -i M_{\Phi_{y}} \\
0 & -i M_{\Phi_{x}} & i M_{\Phi_{y}} & 0
\end{array}\right)
$$

and so

$$
\begin{array}{lll}
J_{c}=\left(\begin{array}{ll}
1 & 0 \\
0 & j_{c}
\end{array}\right), & \text { where } & j_{c}=\frac{1}{\sqrt{2}}\left(\begin{array}{ccc}
c_{c} & -c_{c} & i s_{c} \sqrt{2} \\
-s_{c} & s_{c} & i c_{c} \sqrt{2} \\
i & i & 0
\end{array}\right) \\
c_{c}=\frac{1}{\sqrt{\left(M_{\Phi_{y}} / M_{\Phi_{x}}\right)^{2}+1}} & s_{c}=\frac{1}{\sqrt{\left(M_{\Phi_{x}} / M_{\Phi_{y}}\right)^{2}+1}}
\end{array}
$$

Note that for $M_{\Phi_{x}}=M_{\Phi_{y}}$ we have $c_{c}=s_{c}=1 / \sqrt{2}$, and so the rotation matrix (A.42) becomes flux-independent. Similarly to the previous sectors, we now have that

$$
\begin{aligned}
& \left(\begin{array}{l}
\mathcal{D}_{x} \\
\mathcal{D}_{y} \\
\mathcal{D}_{z}
\end{array}\right)=j_{c}^{t}\left(\begin{array}{c}
D_{x} \\
D_{y} \\
D_{z}
\end{array}\right)=\frac{1}{\sqrt{2}}\left(\begin{array}{c}
c_{c} \partial_{x}-s_{c} \partial_{y} \pm \sqrt{2} M_{\Phi}\left(c_{c} \bar{x}-s_{c} \bar{y}\right) \\
-c_{c} \partial_{x}+s_{c} \partial_{y} \pm \sqrt{2} M_{\Phi}\left(c_{c} \bar{x}-s_{c} \bar{y}\right) \\
i \sqrt{2}\left(s_{c} \partial_{x}+c_{c} \partial_{y}\right)
\end{array}\right) \\
& \left(\begin{array}{c}
\mathcal{D}_{\bar{x}} \\
\mathcal{D}_{\bar{y}} \\
\mathcal{D}_{\bar{z}}
\end{array}\right)=j_{c}^{-1}\left(\begin{array}{c}
D_{\bar{x}} \\
D_{\bar{y}} \\
D_{\bar{z}}
\end{array}\right)=\frac{1}{\sqrt{2}}\left(\begin{array}{c}
c_{s} \partial_{\bar{x}}-s_{c} \partial_{\bar{y}} \mp \sqrt{2} M_{\Phi}\left(c_{c} x-s_{c} y\right) \\
-c_{c} \partial_{\bar{x}}+s_{c} \partial_{\bar{y}} \mp \sqrt{2} M_{\Phi}\left(c_{c} x-s_{c} y\right) \\
-i \sqrt{2}\left(s_{c} \partial_{\bar{x}}+c_{c} \partial_{\bar{y}}\right)
\end{array}\right)
\end{aligned}
$$


for the $c^{ \pm}$sectors respectively, and where we have defined $M_{\Phi}=\sqrt{M_{\Phi_{x}}^{2}+M_{\Phi_{y}}^{2}} / \sqrt{2}$. Finally,

$$
\mathcal{D}_{\mathbf{A}}^{\dagger} \mathcal{D}_{\mathbf{A}}=J_{c}^{-1} \mathbf{D}_{\mathbf{A}}^{\dagger} \mathbf{D}_{\mathbf{A}} J_{c}=-\Delta_{c^{ \pm}} \mathbb{I}_{4} \pm \sqrt{2} M_{\Phi}\left(\begin{array}{cccc}
0 & 0 & 0 & 0 \\
0 & -1 & 0 & 0 \\
0 & 0 & 1 & 0 \\
0 & 0 & 0 & 0
\end{array}\right)
$$

Unlike the previous sectors, the sector $c$ is non-chiral, meaning that one finds zero mode solutions both in the $c^{+}$and the $c^{-}$sectors. Indeed, such zero modes are given by

$$
\Psi_{0, c^{+}}=J_{c}\left(\begin{array}{l}
0 \\
1 \\
0 \\
0
\end{array}\right) \psi_{0, c}, \quad \Psi_{0, c^{-}}=J_{c}\left(\begin{array}{l}
0 \\
0 \\
1 \\
0
\end{array}\right) \psi_{0, c}, \quad \psi_{0, c}=e^{-\sqrt{2} M_{\Phi}\left|c_{c} x-s_{c} y\right|^{2}}
$$

with the scalar wavefunction $\psi_{0, c}$ satisfying

$$
\mathcal{D}_{x} \psi_{0, c}=\mathcal{D}_{\bar{y}} \psi_{0, c}=\mathcal{D}_{\bar{z}} \psi_{0, c}=0
$$

in the $c^{+}$sector and

$$
\mathcal{D}_{y} \psi_{0, c}=\mathcal{D}_{\bar{x}} \psi_{0, c}=\mathcal{D}_{\bar{z}} \psi_{0, c}=0
$$

in the $c^{-}$sector. The commutation relations of these sectors are given by

$$
\begin{aligned}
{\left[\Delta_{c^{ \pm}}, \mathcal{D}_{x}\right] } & = \pm \sqrt{2} M_{\Phi} \mathcal{D}_{x} & {\left[\Delta_{c^{ \pm}}, \mathcal{D}_{\bar{x}}\right] } & =\mp \sqrt{2} M_{\Phi} \mathcal{D}_{\bar{x}} \\
{\left[\Delta_{c^{ \pm}}, \mathcal{D}_{y}\right] } & =\mp \sqrt{2} M_{\Phi} \mathcal{D}_{y} & {\left[\Delta_{c^{ \pm}}, \mathcal{D}_{\bar{y}}\right] } & = \pm \sqrt{2} M_{\Phi} \mathcal{D}_{\bar{y}} \\
{\left[\Delta_{c^{ \pm}}, \mathcal{D}_{z}\right] } & =0 & {\left[\Delta_{c^{ \pm}}, \mathcal{D}_{\bar{z}}\right] } & =0
\end{aligned}
$$

so that we can build the eigenfunctions of, e.g., $\Delta_{c^{+}}$by applying the creator operators $\mathcal{D}_{\bar{x}}$ and $\mathcal{D}_{y}$, and so construct the spectrum of massive modes in the $c^{+}$sector up to the Kaluza-Klein excitations along the direction $x+y$.

\section{A.2 Fermionic conventions}

In order to describe explicitly fermionic wavefunctions we take the following representation for $\Gamma$-matrices in flat $10 \mathrm{~d}$ space

$$
\Gamma^{\underline{\mu}}=\gamma^{\mu} \otimes \mathbb{I}_{2} \otimes \mathbb{I}_{2} \otimes \mathbb{I}_{2} \quad \Gamma^{\underline{m}}=\gamma_{(4)} \otimes \tilde{\gamma}^{m-3}
$$

where $\mu=0, \ldots, 3$, labels the $4 \mathrm{~d}$ Minkowski coordinates, whose gamma matrices are

$$
\gamma^{0}=\left(\begin{array}{cc}
0 & -\mathbb{I}_{2} \\
\mathbb{I}_{2} & 0
\end{array}\right) \quad \gamma^{i}=\left(\begin{array}{cc}
0 & \sigma_{i} \\
\sigma_{i} & 0
\end{array}\right)
$$

$m=4, \ldots, 9$ labels the extra $\mathbb{R}^{6}$ coordinates

$$
\begin{aligned}
& \tilde{\gamma}^{1}=\sigma_{1} \otimes \mathbb{I}_{2} \otimes \mathbb{I}_{2} \quad \tilde{\gamma}^{4}=\sigma_{2} \otimes \mathbb{I}_{2} \otimes \mathbb{I}_{2} \\
& \tilde{\gamma}^{2}=\sigma_{3} \otimes \sigma_{1} \otimes \mathbb{I}_{2} \quad \tilde{\gamma}^{5}=\sigma_{3} \otimes \sigma_{2} \otimes \mathbb{I}_{2} \\
& \tilde{\gamma}^{3}=\sigma_{3} \otimes \sigma_{3} \otimes \sigma_{1} \quad \tilde{\gamma}^{6}=\sigma_{3} \otimes \sigma_{3} \otimes \sigma_{2}
\end{aligned}
$$


and $\sigma_{i}$ indicate the usual Pauli matrices. The $4 \mathrm{~d}$ chirality operator is then given by

$$
\Gamma_{(4)}=\gamma_{(4)} \otimes \mathbb{I}_{2} \otimes \mathbb{I}_{2} \otimes \mathbb{I}_{2}
$$

where $\gamma_{(4)}=i \gamma^{0} \gamma^{1} \gamma^{2} \gamma^{3}$, and the 10d chirality operator by

$$
\Gamma_{(10)}=\gamma_{(4)} \otimes \gamma_{(6)}=\left(\begin{array}{cc}
\mathbb{I}_{2} & 0 \\
0 & -\mathbb{I}_{2}
\end{array}\right) \otimes \sigma_{3} \otimes \sigma_{3} \otimes \sigma_{3}
$$

with $\gamma_{(6)}=-i \tilde{\gamma}^{1} \tilde{\gamma}^{2} \tilde{\gamma}^{3} \tilde{\gamma}^{4} \tilde{\gamma}^{5} \tilde{\gamma}^{6}$. Finally, in this choice of representation a Majorana matrix is given by

$$
\mathcal{B}=\Gamma^{\underline{2}} \Gamma^{\underline{7}} \Gamma^{\underline{8}} \Gamma^{\underline{9}}=\left(\begin{array}{cc}
0 & \sigma_{2} \\
-\sigma_{2} & 0
\end{array}\right) \otimes \sigma_{2} \otimes i \sigma_{1} \otimes \sigma_{2}=\mathcal{B}_{4} \otimes \mathcal{B}_{6}
$$

which indeed satisfies the conditions $\mathcal{B B}^{*}=\mathbb{I}$ and $\mathcal{B} \Gamma \underline{\underline{M}} \mathcal{B}^{*}=\Gamma^{\underline{M}} *$. Notice that the $4 \mathrm{~d}$ and 6d Majorana matrices $\mathcal{B}_{4} \equiv \gamma^{2} \gamma_{(4)}$ and $\mathcal{B}_{6} \equiv \tilde{\gamma}^{4} \tilde{\gamma}^{5} \tilde{\gamma}^{6}$ satisfy analogous conditions $\mathcal{B}_{4} \mathcal{B}_{4}^{*}=\mathcal{B}_{6} \mathcal{B}_{6}^{*}=\mathbb{I}$ and $\mathcal{B}_{4} \gamma^{\mu} \mathcal{B}_{4}^{*}=\gamma^{\mu *}, \mathcal{B}_{6} \gamma^{m} \mathcal{B}_{6}^{*}=-\gamma^{m *}$.

In the text we mainly work with 10d Majorana-Weyl spinors of negative chirality, meaning those spinors $\theta$ satisfying $\theta=-\Gamma_{(10)} \theta=\mathcal{B}^{*} \theta^{*}$. In the conventions above this means that we have spinors of the form

$$
\begin{aligned}
& \theta^{0}=\psi^{0}\left(\begin{array}{c}
\xi_{+} \\
0
\end{array}\right) \otimes \chi_{---}+i\left(\psi^{0}\right)^{*}\left(\begin{array}{c}
0 \\
\sigma_{2} \xi_{+}^{*}
\end{array}\right) \otimes \chi_{+++} \\
& \theta^{1}=\psi^{1}\left(\begin{array}{c}
\xi_{+} \\
0
\end{array}\right) \otimes \chi_{-++}-i\left(\psi^{1}\right)^{*}\left(\begin{array}{c}
0 \\
\sigma_{2} \xi_{+}^{*}
\end{array}\right) \otimes \chi_{+--} \\
& \theta^{2}=\psi^{2}\left(\begin{array}{c}
\xi_{+} \\
0
\end{array}\right) \otimes \chi_{+-+}+i\left(\psi^{2}\right)^{*}\left(\begin{array}{c}
0 \\
\sigma_{2} \xi_{+}^{*}
\end{array}\right) \otimes \chi_{-+-} \\
& \theta^{3}=\psi^{3}\left(\begin{array}{c}
\xi_{+} \\
0
\end{array}\right) \otimes \chi_{++-}-i\left(\psi^{3}\right)^{*}\left(\begin{array}{c}
0 \\
\sigma_{2} \xi_{+}^{*}
\end{array}\right) \otimes \chi_{--+}
\end{aligned}
$$

where $\psi^{j}$ is the spinor wavefunction, $\left(\xi_{+} 0\right)^{t}$ is a $4 \mathrm{~d}$ spinor of positive chirality and $\chi_{\epsilon_{1} \epsilon_{2} \epsilon_{3}}$ is a basis of $6 \mathrm{~d}$ spinors of such that

$$
\chi_{---}=\left(\begin{array}{l}
0 \\
1
\end{array}\right) \otimes\left(\begin{array}{l}
0 \\
1
\end{array}\right) \otimes\left(\begin{array}{l}
0 \\
1
\end{array}\right) \quad \chi_{+++}=\left(\begin{array}{l}
1 \\
0
\end{array}\right) \otimes\left(\begin{array}{l}
1 \\
0
\end{array}\right) \otimes\left(\begin{array}{l}
1 \\
0
\end{array}\right)
$$

etc. Note that these basis elements are eigenstates of the $6 \mathrm{~d}$ chirality operator $\gamma_{(6)}$, with eigenvalues $\epsilon_{1} \epsilon_{2} \epsilon_{3}$. In (A.56), we need to replace $\left(\psi^{m}\right)^{*} \rightarrow\left(\psi^{m}\right)^{\dagger}$ if the spinor $\theta$ transforms in the adjoint of a gauge group.

Finally, let us recall that to dimensionally reduce a 10d fermionic action, one has to simultaneously diagonalize two Dirac operators: $\not_{\mathbb{R}^{1,3}}$ and $\not D^{\text {int }}$, built from $\Gamma^{\underline{\mu}}$ and $\Gamma^{\underline{m}}$, respectively. However, as these two set of $\Gamma$-matrices do not commute, nor will $\not_{\mathbb{R}^{1,3}}$ and $\not D^{\text {int }}$, and so we need instead to construct these Dirac operators from the alternative $\Gamma$-matrices

$$
\tilde{\Gamma}^{\underline{\mu}}=\Gamma_{(4)} \Gamma^{\underline{\mu}}=\Gamma_{(4)} \gamma^{\mu} \otimes \mathbb{I}_{2} \otimes \mathbb{I}_{2} \otimes \mathbb{I}_{2} \quad \tilde{\Gamma}^{\underline{m}}=\Gamma_{(4)} \Gamma^{\underline{m}}=\mathbb{I}_{4} \otimes \tilde{\gamma}^{m-3}
$$

following the common practice in the literature. 


\section{B Computing wavefunctions in the commutative formalism}

In this appendix we address the computation of the corrected zero mode wavefunctions in the commutative $8 \mathrm{~d}$ formalism described in susbsection 3.2. In particular, we will look for a corrected wavefunction of the form (4.1), with $\left\{\Psi_{\lambda}\right\}$ the tower of unperturbed massive modes at the matter curve (i.e., those wavefunctions satisfying eq. (2.11)).

\section{B.1 $\beta$-deformed wavefunctions and massive modes}

As already discussed in section 4 , the zero mode wavefunctions in the presence of a nonperturbative effect or a $\beta$-deformation can be expressed in terms of the expansion

$$
\Psi=\Psi^{(0)}+\epsilon \Psi^{(1)}+\epsilon^{2} \Psi^{(2)}+\ldots
$$

where $\Psi^{(0)}=\Psi_{0}$ is the wavefunction in the absence of the non-perturbative effect $/ \beta$ deformation, satisfying the classical equation (4.2). The equation for the first order correction $\Psi^{(1)}$ is given by

$$
\mathbf{D}_{\mathbf{A}} \Psi^{(1)}=\mathbf{K}^{(1)} \Psi_{0}
$$

where $\mathbf{K}^{(1)}$ is the following linear operator

$$
\begin{aligned}
& \mathbf{K}^{(1)}=-\left(\begin{array}{cc}
0 & 0 \\
0 & \Theta(\tilde{\mathbf{K}}+i \tilde{\mathbf{A}})^{(0)} \Theta
\end{array}\right) \\
& -(\tilde{\mathbf{K}}+i \tilde{\mathbf{A}})=\left(\begin{array}{ccc}
0 & \mathcal{K}_{\bar{z}}+i \mathcal{A}_{\bar{z}} & -\left(\mathcal{K}_{\bar{y}}+\frac{i}{2} \mathcal{A}_{\bar{y}}\right) \\
-\left(\mathcal{K}_{\bar{z}}+i \mathcal{A}_{\bar{z}}\right) & 0 & \mathcal{K}_{\bar{x}}+\frac{i}{2} \mathcal{A}_{\bar{x}} \\
\mathcal{K}_{\bar{y}}+\frac{i}{2} \mathcal{A}_{\bar{y}} & -\left(\mathcal{K}_{\bar{x}}+\frac{i}{2} \mathcal{A}_{\bar{x}}\right) & 0
\end{array}\right) \\
& \mathcal{K}_{\bar{m}}=\left\{F_{y \bar{m}}^{\theta}, D_{x} \cdot\right\}-\left\{F_{x \bar{m}}^{\theta}, D_{y} \cdot\right\} \quad \mathcal{A}_{\bar{m}}=\left[\left\langle F_{y \bar{m}}^{\theta} A_{x}-F_{x \bar{m}}^{\theta} A_{y}\right\rangle, \cdot\right] \\
& F_{l \bar{n}}^{\theta}=\left\langle F_{l \bar{n}}\right\rangle \quad F_{l \bar{z}}^{\theta}=\partial_{x}\left(\theta\left\langle\Phi_{x y}\right\rangle\right) \quad l, n=x, y
\end{aligned}
$$

From the results of subsection 3.1, one expects that the solution to (B.2) is given by a linear combination of massive modes of the unperturbed system. That is, $\Psi^{(1)}$ should be of the form

$$
\Psi^{(1)}=\sum_{\lambda} c_{\lambda} \Psi_{\lambda}
$$

with $c_{\lambda} \in \mathbb{C}$ and each $\Psi_{\lambda}$ satisfying

$$
\mathbf{D}_{\mathbf{A}}^{\dagger} \mathbf{D}_{\mathbf{A}} \Psi_{\lambda}=\left|m_{\lambda}\right|^{2} \Psi_{\lambda}
$$

Hence, using the eigenmode decomposition (B.4) we obtain that eq. (B.2) can be expressed as

$$
\mathbf{D}_{\mathbf{A}}^{\dagger} \mathbf{K}^{(1)} \Psi_{0}=\sum_{\lambda} c_{\lambda}\left|m_{\lambda}\right|^{2} \Psi_{\lambda}
$$

for some set of coefficients $c_{\lambda}$ to be found for each different $\Psi_{0}$. The corrected wavefunction will then be given by

$$
\Psi=\Psi_{0}+\epsilon \sum_{\lambda} c_{\lambda} \Psi_{\lambda}+\mathcal{O}\left(\epsilon^{2}\right)
$$

In the following, we will illustrate the above method by computing the corrected wavefunctions for the $\mathrm{U}(3)$ toy model of section 2.3 , for the particular case of constant $\theta$. 


\section{B.2 The U(3) model}

Let us again consider the $\mathrm{U}(3)$ model of subsection 2.3, together with its full spectrum of massive modes worked out in appendix A. In particular, recall from that appendix that the classical massive modes $\Psi_{\lambda}$ of a model with constant fluxes are given by (A.13), for some specific rotation matrix $J$ that differs for each sector, and that the scalar wavefunctions $\psi_{\rho}$ are related to the zero mode wavefunction $\psi_{0}$ by means of the creation operators $\mathcal{D}_{m}$ defined in (A.16). Hence, one may solve eq. (B.6) by expressing $\mathbf{D}_{\mathbf{A}}{ }^{\dagger} \mathbf{K}^{(1)}$ in terms of such creator operators. We will show how to do so for each of the chiral sectors of the $\mathrm{U}(3)$ model, restricting for simplicity to the case where $\theta$ is a constant.

\section{Sector $a^{+}$}

For this sector and for constant $\theta$ it is easy to see that

$$
\begin{aligned}
\Theta \tilde{\mathbf{K}} \Theta & =\frac{i \theta}{6}\left(\begin{array}{ccc}
0 & i m_{\Phi}^{2}\left(D_{y}+2 D_{x}\right) & -M_{y} D_{x} \\
-i m_{\Phi}^{2}\left(D_{y}+2 D_{x}\right) & 0 & -M_{x} D_{y} \\
M_{y} D_{x} & M_{x} D_{y} & 0
\end{array}\right) \\
i \Theta \tilde{\mathbf{A}} \Theta & =\frac{i \theta}{6}\left(\begin{array}{ccc}
0 & -2 m_{\Phi}^{2} \overline{A_{x}} & -i M_{y} \overline{A_{x}} \\
2 m_{\Phi}^{2} \overline{A_{x}} & 0 & -i M_{x} \overline{A_{y}} \\
i M_{y} \overline{A_{x}} & i M_{x} \overline{A_{y}} & 0
\end{array}\right)
\end{aligned}
$$

where $\bar{A}_{x}$ and $\bar{A}_{y}$ are defined as in (2.28). Since for the sector $a^{+}$we have that $D_{x}=\partial_{x}-i \bar{A}_{x}$ and $D_{y}=\partial_{y}-i \bar{A}_{y}$, we obtain

$$
\mathbf{K}_{a^{+}}^{(1)}=\frac{i \theta}{6}\left(\begin{array}{cccc}
0 & 0 & 0 & 0 \\
0 & 0 & -i m_{\Phi}^{2}\left(D_{y}+2 \partial_{x}\right) & M_{y} \partial_{x} \\
0 i m_{\Phi}^{2}\left(D_{y}+2 \partial_{x}\right) & 0 & M_{x} \partial_{y} \\
0 & -M_{y} \partial_{x} & -M_{x} \partial_{y} & 0
\end{array}\right)
$$

Recall that the classical zero modes for the sector $a^{+}$in the holomorphic gauge can be expressed either as (2.29) or as (A.26). One then deduces that

$$
\mathbf{D}_{\mathbf{A}^{\dagger}} \mathbf{K}_{a^{+}}^{(1)} \Psi_{0, a^{+}}=\mathcal{P}_{a} \Psi_{0, a^{+}}=\left(\begin{array}{c}
0 \\
-i \frac{\lambda_{a}^{-}}{m_{\Phi}^{2}} \\
0 \\
1
\end{array}\right) \mathcal{P}_{a} \psi_{0, a^{+}}
$$

with the differential operator $\mathcal{P}_{a}$ given by

$$
\mathcal{P}_{a}=\frac{i \theta}{6}\left[\left(2 s_{a}^{2} \lambda_{a}^{-}\right) \mathcal{D}_{x}^{2}+\left(c_{a}\left(\lambda_{a}^{-}+2 M_{x y}\right)-M_{y} / c_{a}\right) \mathcal{D}_{x} \mathcal{D}_{y}+i c_{a} M_{x} \overline{A_{y}} \mathcal{D}_{x}\right]
$$

and where, following the definitions of appendix A, $\lambda_{a}^{ \pm}$is defined as in (2.25) and

$$
\begin{aligned}
\mathcal{D}_{x}=c_{a}\left(\partial_{x}-\lambda_{a}^{+} \bar{x}\right) & \mathcal{D}_{y}=\partial_{y}-M_{y} \bar{y} \\
c_{a}=\frac{m_{\Phi}^{2}}{\sqrt{\left(\lambda_{a}^{-}\right)^{2}+m_{\Phi}^{4}}} & s_{a}=\frac{\lambda_{a}^{-}}{\sqrt{\left(\lambda_{a}^{-}\right)^{2}+m_{\Phi}^{4}}}
\end{aligned}
$$


Notice that in (B.11) the terms proportional to $\psi_{200, a^{+}} \equiv \mathcal{D}_{x}^{2} \psi_{0, a^{+}}$and $\psi_{110, a^{+}} \equiv \mathcal{D}_{x} \mathcal{D}_{y} \psi_{0, a^{+}}$ are already of the form (B.6), for these are eigenfunctions of the Laplace operator $\Delta$. The same is not true for the term proportional to $\overline{A_{y}} \mathcal{D}_{x}$. Indeed, this term is difficult to express purely in terms of creation operators $\mathcal{D}_{m}$, since $\bar{A}_{y}$ depends on the coordinate $\bar{y}$ along the matter curve. In order to circumvent this difficulty one may choose a gauge for $\langle A\rangle$ slightly different from the one implicit in (3.45). Such gauge $\left\langle A^{\prime}\right\rangle$ is defined by

$$
\left\langle A^{\prime}\right\rangle=\langle A\rangle+\mathrm{d} \Omega \quad \rightarrow \quad \Psi^{\prime}=e^{i \Omega} \Psi \quad \Omega=\frac{\epsilon \theta}{2}\left\langle A_{x} A_{y}\right\rangle^{(0)}
$$

In this new gauge we have that (B.9) is replaced by

$$
i \Theta \tilde{\mathbf{A}}^{\prime} \Theta=\frac{i \theta}{6}\left(\begin{array}{ccc}
0 & -2 m_{\Phi}^{2} \overline{A_{x}}-2 i M_{y} \overline{A_{x}} \\
2 m_{\Phi}^{2} \overline{A_{x}} & 0 & 0 \\
2 i M_{y} \overline{A_{x}} & 0 & 0
\end{array}\right)
$$

and so instead of (B.11) we have

$$
\mathbf{D}_{\mathbf{A}}^{\dagger}\left(\mathbf{K}_{a^{+}}^{(1)}\right)^{\prime} \Psi_{0, a^{+}}^{\prime}=\mathcal{P}_{a}^{\prime} \Psi_{0, a^{+}}^{\prime}
$$

where

$$
\mathcal{P}_{a}^{\prime}=\frac{i \theta}{6} s_{a} m_{\Phi}^{2}\left[\left(2 s_{a}^{2} / c_{a}\right) \mathcal{D}_{x}^{2}+\left(1+\frac{2 M_{x y} c_{a}}{m_{\Phi}^{2} s_{a}}-\frac{2 M_{y} s_{a}}{m_{\Phi}^{2} c_{a}}\right) \mathcal{D}_{x} \mathcal{D}_{y}\right]
$$

Hence, in this gauge we have that

$$
\mathbf{D}_{\mathbf{A}^{\dagger}}^{\dagger}\left(\mathbf{K}_{a^{+}}^{(1)}\right)^{\prime} \Psi_{0, a^{+}}^{\prime}=\frac{i \theta}{6} m_{\Phi}^{2} s_{a}\left[\frac{2 s_{a}^{2}}{c_{a}} \Psi_{200, a^{+}}+\left(1+\frac{2 M_{x y} c_{a}}{m_{\Phi}^{2} s_{a}}-\frac{2 M_{y} s_{a}}{m_{\Phi}^{2} c_{a}}\right) \Psi_{110, a^{+}}\right]
$$

where $\Psi_{200, a^{+}}=\mathcal{D}_{x}^{2} \Psi_{0, a^{+}}$and $\Psi_{110, a^{+}}=\mathcal{D}_{x} \mathcal{D}_{y} \Psi_{0, a^{+}}$are massive replicas of the chiral mode $\Psi_{0, a^{+}}$, of masses $\left|m_{200}\right|^{2}=2 \lambda_{a}^{+}=-2 m_{\Phi}^{2} c_{a} / s_{a}$ and $\left|m_{110}\right|^{2}=\left(M_{y}+\lambda_{a}^{+}\right)=\left(2 M_{x y}-\right.$ $m_{\Phi}^{2} s_{a} / c_{a}$ ), respectively (see table 1 in appendix A). By plugging the above expression into (B.7) we obtain that the coefficients $c_{\lambda}$ are

$$
c_{200}=-\frac{i \theta}{6} \frac{s_{a}^{4}}{c_{a}^{2}} \quad \text { and } \quad c_{110}=-\frac{i \theta}{6}\left(\frac{2 M s_{a}}{m_{\Phi}^{2}}+c_{a}\right)
$$

where for simplicity we have set $M \equiv M_{x}=-M_{y}$. We finally conclude that the corrected wavefunction for the $a^{+}$sector is given by

$$
\begin{aligned}
\Psi_{a^{+}} & =\Psi_{0, a^{+}}-\frac{i \epsilon \theta}{6}\left[\frac{s_{a}^{4}}{c_{a}^{2}} \Psi_{200, a^{+}}+\left(2 M s_{a} / m_{\Phi}^{2}+c_{a}\right) \Psi_{110, a^{+}}-\bar{A}_{x} \bar{A}_{y} \Psi_{0, a^{+}}\right]+\mathcal{O}\left(\epsilon^{2}\right) \\
& =\left(\begin{array}{c}
0 \\
-i \frac{\lambda_{a}^{-}}{m_{\Phi}^{2}} \\
0 \\
1
\end{array}\right)\left[1-\frac{i \epsilon \theta}{6}\left(\frac{s_{a}^{4}}{c_{a}^{2}} \mathcal{D}_{x}^{2}+\left(2 M s_{a} / m_{\Phi}^{2}+c_{a}\right) \mathcal{D}_{x} \mathcal{D}_{y}-\bar{A}_{x} \bar{A}_{y}\right)\right] \psi_{0, a^{+}}
\end{aligned}
$$

where we have performed the gauge transformation

$$
\Psi_{a^{+}}=e^{-i \Omega} \Psi_{a^{+}}^{\prime}=e^{\frac{i \epsilon \theta}{6} \bar{A}_{x} \bar{A}_{y}} \Psi_{a^{+}}^{\prime}
$$

and expanded it to first order in $\epsilon$. 


\section{Sector $b^{+}$}

For this sector we have

$$
\mathbf{K}_{b^{+}}^{(1)}=\frac{i \theta}{6}\left(\begin{array}{cccc}
0 & 0 & 0 & 0 \\
0 & 0 & i m_{\Phi}^{2}\left(D_{x}+2 \partial_{y}\right) & M_{y} \partial_{x} \\
0-i m_{\Phi}^{2}\left(D_{x}+2 \partial_{y}\right) & 0 & M_{x} \partial_{y} \\
0 & -M_{y} \partial_{x} & -M_{x} \partial_{y} & 0
\end{array}\right)
$$

where for this sector we have $D_{x}=\partial_{x}+i \bar{A}_{x}$ and $D_{y}=\partial_{y}+i \overline{A_{y}}$. Just like in the sector $a^{+}$ we have that eq. (B.6) has the form

$$
\mathbf{D}_{\mathbf{A}^{\dagger}}^{\dagger} \mathbf{K}_{b^{+}}^{(1)} \Psi_{0, b^{+}}=\mathcal{P}_{b} \Psi_{0, b^{+}}
$$

with

$$
\begin{aligned}
& \mathcal{P}_{b}=\frac{i \theta}{6}\left[\left(2 s_{b}^{2} \lambda_{b}^{-}\right) \mathcal{D}_{y}^{2}+\left(c_{b}\left(\lambda_{b}^{-}-2 M_{x y}\right)+M_{x} / c_{b}\right) \mathcal{D}_{x} \mathcal{D}_{y}-i c_{b} M_{y} \overline{A_{x}} \mathcal{D}_{y}\right] \\
& \mathcal{D}_{x}=\partial_{x}+M_{x} \bar{x} \quad \mathcal{D}_{y}=c_{b}\left(\partial_{y}-\lambda_{b}^{+} \bar{y}\right)
\end{aligned}
$$

where $\lambda_{b}^{-}$is given by (2.33) and $s_{b}, c_{b}$ are defined as $s_{a}, c_{a}$ with the replacement $\lambda_{a}^{-} \rightarrow \lambda_{b}^{-}$. Note that the operators (B.12) and (B.25) are basically related by the exchange $x \leftrightarrow y$, and so one may already guess that the same will be true for the corrected wavefunctions.

Just like in the sector $a^{+}$, expressing the term $\bar{A}_{x} \mathcal{D}_{y}$ in term of creation operators is difficult, so one may take a different gauge for $\langle A\rangle$. The appropriate choice is again (B.15) but with the replacement $\Omega \rightarrow-\Omega$. We then find

$$
\mathbf{D}_{\mathbf{A}}^{\dagger}\left(\mathbf{K}_{b^{+}}^{(1)}\right)^{\prime} \Psi_{0, b^{+}}^{\prime}=\frac{i \theta}{6} m_{\Phi}^{2} s_{b}\left[\frac{2 s_{b}^{2}}{c_{b}} \Psi_{020, b^{+}}+\left(1-\frac{2 M_{x y} c_{b}}{m_{\Phi}^{2} s_{b}}+\frac{2 M_{x} s_{b}}{m_{\Phi}^{2} c_{b}}\right) \Psi_{110, b^{+}}\right]
$$

where $\Psi_{020, b^{+}}=\mathcal{D}_{y}^{2} \Psi_{0, b^{+}}$and $\Psi_{110, b^{+}}=\mathcal{D}_{x} \mathcal{D}_{y} \Psi_{0, b^{+}}$correspond to massive replicas with masses $\left|m_{020}\right|^{2}=2 \lambda_{b}^{+}=-2 m_{\Phi}^{2} c_{b} / s_{b}$ and $\left|m_{110}\right|^{2}=\lambda_{b}^{+}-M_{x}=-\left(2 M_{x y}+m_{\Phi}^{2} s_{b} / c_{b}\right)$, respectively. The coefficients $c_{\lambda}$ in (B.6) now read

$$
c_{020}=-\frac{i \theta}{6} \frac{s_{b}^{4}}{c_{b}^{2}} \quad \text { and } \quad c_{110}=-\frac{i \theta}{6}\left(\frac{2 M s_{b}}{m_{\Phi}^{2}}+c_{b}\right)
$$

where again we have set $M \equiv M_{x}=-M_{y}$. Hence

$$
\begin{aligned}
\Psi_{b^{+}} & =\Psi_{0, b^{+}}-\frac{i \epsilon \theta}{6}\left[\frac{s_{b}^{4}}{c_{b}^{2}} \Psi_{020, b^{+}}+\left(2 M s_{b} / m_{\Phi}^{2}+c_{b}\right) \Psi_{110, a^{+}}-\overline{A_{x}} \overline{A_{y}} \Psi_{0, b^{+}}\right]+\mathcal{O}\left(\epsilon^{2}\right) \\
& =\left(\begin{array}{c}
0 \\
0 \\
i \frac{\lambda_{-}}{m_{\Phi}^{2}} \\
1
\end{array}\right)\left[1-\frac{i \epsilon \theta}{6}\left(\frac{s_{b}^{4}}{c_{b}^{2}} \mathcal{D}_{y}^{2}+\left(2 M s_{b} / m_{\Phi}^{2}+c_{b}\right) \mathcal{D}_{x} \mathcal{D}_{y}-\overline{A_{x}} \overline{A_{y}}\right)\right] \psi_{0, b^{+}}
\end{aligned}
$$

where now

$$
\Psi_{b^{+}}=e^{i \Omega} \Psi_{b^{+}}^{\prime}=e^{\frac{i \epsilon \theta}{6} \bar{A}_{x} \bar{A}_{y}} \Psi_{b^{+}}^{\prime}
$$




\section{Sector $c^{+}$}

For this sector we have

$$
\begin{aligned}
& \Theta \tilde{\mathbf{K}} \Theta=\frac{i \theta}{6}\left(\begin{array}{ccc}
0 & -i m_{\Phi}^{2}\left(\partial_{x}-\partial_{y}\right) & 2 M_{y} \partial_{x} \\
i m_{\Phi}^{2}\left(\partial_{x}-\partial_{y}\right) & 0 & 2 M_{x} \partial_{y} \\
-2 M_{y} \partial_{x} & -2 M_{x} \partial_{y} & 0
\end{array}\right) \\
& \Theta \tilde{\mathbf{A}} \Theta=\frac{i \theta}{6}\left(\begin{array}{ccc}
0 & 2 m_{\Phi}^{2}\left(\overline{A_{x}}+\overline{A_{y}}\right) & 0 \\
-2 m_{\Phi}^{2}\left(\overline{A_{x}}+\overline{A_{y}}\right) & 0 & 0 \\
0 & 0 & 0
\end{array}\right)
\end{aligned}
$$

from which we deduce that

$$
\mathbf{D}_{\mathbf{A}^{\dagger}}^{\dagger} \mathbf{K}_{c^{+}}^{(1)} \Psi_{0, c^{+}}=\mathcal{P}_{c} \Psi_{0, c^{+}}
$$

with

$$
\begin{aligned}
& \mathcal{P}_{c}=\frac{i \theta}{6}\left[\left(M_{x}-M_{y}-\frac{m_{\Phi}^{2}}{\sqrt{2}}\right) \mathcal{D}_{y}^{2}+m_{\Phi}^{2} M_{x y}(\bar{x}+\bar{y}) \mathcal{D}_{y}\right] \\
& \mathcal{D}_{y}=\frac{1}{2}\left(-\partial_{x}+\partial_{y}+\sqrt{2} m_{\Phi}^{2}(\bar{x}-\bar{y})\right)
\end{aligned}
$$

Note that we also find a non-trivial dependence of $\mathcal{P}_{c}$ along the coordinate $\bar{x}+\bar{y}$ along the matter curve $\Sigma_{c}$. This time, however, such term vanishes if we set $M \equiv M_{x}=$ $-M_{y}$, and then the correction only involves the massive mode $\Psi_{020, c^{+}}=\mathcal{D}_{y}^{2} \Psi_{0, c^{+}}$, of mass $\left|m_{020}\right|=2 \sqrt{2} m_{\Phi}^{2}$. In particular, from (B.34) we can deduce the coefficient $c_{020}$ and so

$$
\begin{aligned}
\Psi_{c^{+}} & =\Psi_{0, c^{+}}-\frac{i \epsilon \theta}{6}\left[\frac{1}{4}-\frac{M}{\sqrt{2} m_{\Phi}^{2}}\right] \Psi_{020, c^{+}}+\mathcal{O}\left(\epsilon^{2}\right) \\
& =\left(\begin{array}{c}
0 \\
-\frac{i}{\sqrt{2}} \\
\frac{i}{\sqrt{2}} \\
1
\end{array}\right)\left[1-\frac{i \epsilon \theta}{6}\left(\frac{1}{4}-\frac{M}{\sqrt{2} m_{\Phi}^{2}}\right) \mathcal{D}_{y}^{2}\right] \psi_{0, c^{+}}+\mathcal{O}\left(\epsilon^{2}\right)
\end{aligned}
$$

\section{Equivalence of commutative and non-commutative actions}

As shown in the main text, computing non-perturbative effects on 7-brane wavefunctions is equivalent to compute wavefunctions for either 7 -branes in a $\beta$-deformed background, or for a non-commutative version of the 7-brane action, at least up to $\mathcal{O}\left(\epsilon^{2}\right)$ corrections. That the last two approaches are equivalent could have been guessed from the results of [61, 62], where it was argued that on a D-brane world-volume the effect of a $\beta$-deformation can be seen as a non-commutative deformation of the gauge theory. This point was made more precise in [23] where it was shown, via a Seiberg-Witten map, the equivalence between a $\beta$-deformed, commutative 7 -brane action and a non-commutative 7-brane action.

The purpose of this appendix is to reproduce in detail the derivation in [23] of the equivalence of such commutative and non-commutative 7-brane actions, by means of a slightly simpler Seiberg-Witten map that will also be used in section 4.2. 
Let us consider a non-commutative 7-brane action with non-commutative parameter $\Theta^{x y}=-\Theta^{y x}=-\epsilon \theta$, with $\epsilon$ constant and $\theta$ holomorphic on $(x, y)$, and all the remaining components of $\Theta^{i j}$ vanishing. Such action is given by

$$
\hat{W}=m_{*}^{4} \int_{S} \operatorname{Tr}(\hat{\Phi} \circledast \hat{F})=m_{*}^{4} \int_{S} \operatorname{Tr}(\hat{\Phi} \wedge \hat{F})+\mathcal{O}\left(\epsilon^{2}\right)
$$

Let us now define the Seiberg-Witten map

$$
\begin{aligned}
\hat{A}_{\bar{m}} & =A_{\bar{m}}-\frac{1}{2} \Theta^{i j}\left\{A_{i},\left(\partial_{j}+D_{j}\right) A_{\bar{m}}\right\}+\mathcal{O}\left(\epsilon^{2}\right) \\
\hat{\Phi} & =\Phi-\frac{1}{2}\left\{A_{i},\left(\partial_{j}+D_{j}\right)\left(\Theta^{i j} \Phi\right)\right\}+\mathcal{O}\left(\epsilon^{2}\right)
\end{aligned}
$$

Since $\Theta^{i j}$ is holomorphic it commutes with $\partial_{\bar{n}}$ and so, just like in [63], we have that

$$
\hat{F}_{\bar{x} \bar{y}}=F_{\alpha \beta}-\Theta^{i j}\left[\left\{F_{\bar{x} i}, F_{j \bar{y}}\right\}+\frac{1}{2}\left\{A_{i},\left(\partial_{j}+D_{j}\right) F_{\bar{x} \bar{y}}\right]+\mathcal{O}\left(\epsilon^{2}\right)\right.
$$

which together with (C.2b) reproduces eq. (15) of [23] for the case $\theta=$ const. Plugging these two expressions into (C.1) we obtain

$$
\begin{aligned}
m_{*}^{-4} \hat{W}= & \int_{S} \operatorname{Tr}(\Phi \wedge F)-\int_{S} \Theta^{i j} \operatorname{Tr}\left(\Phi_{x y}\left\{F_{\bar{x} i}, F_{j \bar{y}}\right\}\right) \mathrm{dvol}_{S} \\
& -\frac{1}{2} \int_{S} \Theta^{i j} \operatorname{Tr}\left(\Phi_{x y}\left\{A_{i},\left(D_{j}+\partial_{j}\right) F_{\bar{x} \bar{y}}\right\}\right) \mathrm{dvol}_{S} \\
& -\frac{1}{2} \int_{S} \operatorname{Tr}\left(\left\{A_{i},\left(D_{j}+\partial_{j}\right)\left(\Theta^{i j} \Phi_{x y}\right)\right\} F_{\bar{x} \bar{y}}\right) \operatorname{dvol}_{S}+\mathcal{O}\left(\epsilon^{2}\right)
\end{aligned}
$$

We now have that

$$
\begin{aligned}
\frac{1}{2} \operatorname{Tr}\left(\Theta^{i j} \Phi_{x y}\left\{A_{i},\left(D_{j}+\partial_{j}\right) F_{\bar{x} \bar{y}}\right\}+\left\{A_{i},\left(D_{j}+\partial_{j}\right)\left(\Theta^{i j} \Phi_{x y}\right)\right\} F_{\bar{x} \bar{y}}\right) \\
=\Theta^{i j} \operatorname{Tr}\left(\Phi_{x y}\left\{F_{i j}, F_{\bar{x} \bar{y}}\right\}\right)+2 \partial_{j}\left[\Theta^{i j} \operatorname{Tr}\left(A_{i}\left\{\Phi_{x y}, F_{\bar{x} \bar{y}}\right\}\right)\right]
\end{aligned}
$$

and so

$$
\hat{W}=m_{*}^{4}\left[\int_{S} \operatorname{Tr}(\Phi \wedge F)+\frac{\epsilon}{2} \int_{S} \theta \operatorname{Tr}\left(\Phi_{x y} F \wedge F\right)+\int_{S} \partial_{i} \operatorname{Tr}\left[\Theta^{i j}\left(A_{j} \Phi \wedge F\right)\right]\right]+\mathcal{O}\left(\epsilon^{2}\right)
$$

Since $S$ has no boundary and the last term is a total derivative it must vanish, and so we recover the $\beta$-deformed superpotential (3.39).

\section{References}

[1] L.E. Ibáñez and A.M. Uranga, String Theory and Particle Physics. An Introduction to String Phenomenology, Cambridge University Press (2011), to appear.

[2] F. Marchesano and G. Shiu, MSSM vacua from flux compactifications, Phys. Rev. D 71 (2005) 011701 [hep-th/0408059] [SPIRES].

[3] F. Marchesano and G. Shiu, Building MSSM flux vacua, JHEP 11 (2004) 041 [hep-th/0409132] [SPIRES]. 
[4] D. Cremades, L.E. Ibáñez and F. Marchesano, Computing Yukawa couplings from magnetized extra dimensions, JHEP 05 (2004) 079 [hep-th/0404229] [SPIRES].

[5] S.A. Abel and M.D. Goodsell, Realistic Yukawa couplings through instantons in intersecting brane worlds, JHEP 10 (2007) 034 [hep-th/0612110] [SPIRES].

[6] R. Donagi and M. Wijnholt, Model Building with F-theory, arXiv:0802.2969 [SPIRES].

[7] C. Beasley, J.J. Heckman and C. Vafa, GUTs and Exceptional Branes in F-theory - I, JHEP 01 (2009) 058 [arXiv:0802.3391] [SPIRES].

[8] C. Beasley, J.J. Heckman and C. Vafa, GUTs and Exceptional Branes in F-theory - II: Experimental Predictions, JHEP 01 (2009) 059 [arXiv: 0806.0102] [SPIRES].

[9] R. Donagi and M. Wijnholt, Breaking GUT Groups in F-theory, arXiv:0808.2223 [SPIRES].

[10] J.J. Heckman, Particle Physics Implications of F-theory, arXiv:1001.0577 [SPIRES].

[11] T. Weigand, Lectures on F-theory compactifications and model building, Class. Quant. Grav. 27 (2010) 214004 [arXiv:1009.3497] [SPIRES].

[12] A. Font and L.E. Ibáñez, Yukawa Structure from U(1) Fluxes in F-theory Grand Unification, JHEP 02 (2009) 016 [arXiv:0811.2157] [SPIRES].

[13] J.J. Heckman and C. Vafa, Flavor Hierarchy From F-theory, Nucl. Phys. B 837 (2010) 137 [arXiv:0811.2417] [SPIRES].

[14] H. Hayashi, T. Kawano, R. Tatar and T. Watari, Codimension-3 Singularities and Yukawa Couplings in F-theory, Nucl. Phys. B 823 (2009) 47 [arXiv:0901.4941] [SPIRES].

[15] A. Font and L.E. Ibáñez, Matter wave functions and Yukawa couplings in F-theory Grand Unification, JHEP 09 (2009) 036 [arXiv:0907.4895] [SPIRES].

[16] S. Cecotti, M.C.N. Cheng, J.J. Heckman and C. Vafa, Yukawa Couplings in F-theory and Non-Commutative Geometry, arXiv:0910.0477 [SPIRES].

[17] J.P. Conlon and E. Palti, Aspects of Flavour and Supersymmetry in F-theory GUTs, JHEP 01 (2010) 029 [arXiv:0910.2413] [SPIRES].

[18] H. Hayashi, T. Kawano, Y. Tsuchiya and T. Watari, Flavor Structure in F-theory Compactifications, JHEP 08 (2010) 036 [arXiv:0910.2762] [SPIRES].

[19] G. Aldazabal, L.E. Ibáñez, F. Quevedo and A.M. Uranga, D-branes at singularities: A bottom-up approach to the string embedding of the standard model, JHEP 08 (2000) 002 [hep-th/0005067] [SPIRES].

[20] E. Dudas and E. Palti, Froggatt-Nielsen models from E8 in F-theory GUTs, JHEP 01 (2010) 127 [arXiv:0912.0853] [SPIRES].

[21] G.K. Leontaris and G.G. Ross, Yukawa couplings and fermion mass structure in F-theory GUTs, JHEP 02 (2011) 108 [arXiv: 1009.6000] [SPIRES].

[22] S. Krippendorf, M.J. Dolan, A. Maharana and F. Quevedo, D-branes at Toric Singularities: Model Building, Yukawa Couplings and Flavour Physics, JHEP 06 (2010) 092 [arXiv: 1002.1790] [SPIRES].

[23] F. Marchesano and L. Martucci, Non-perturbative effects on seven-brane Yukawa couplings, Phys. Rev. Lett. 104 (2010) 231601 [arXiv:0910.5496] [SPIRES].

[24] D. Baumann, A. Dymarsky, S. Kachru, I.R. Klebanov and L. McAllister, D3-brane Potentials from Fluxes in AdS/CFT, JHEP 06 (2010) 072 [arXiv:1001.5028] [SPIRES]. 
[25] P. Koerber and L. Martucci, From ten to four and back again: how to generalize the geometry, JHEP 08 (2007) 059 [arXiv: 0707.1038] [SPIRES].

[26] A. Collinucci, New F-theory lifts II: Permutation orientifolds and enhanced singularities, JHEP 04 (2010) 076 [arXiv:0906.0003] [SPIRES].

[27] R. Blumenhagen, T.W. Grimm, B. Jurke and T. Weigand, F-theory uplifts and GUTs, JHEP 09 (2009) 053 [arXiv:0906.0013] [SPIRES].

[28] R. Blumenhagen, T.W. Grimm, B. Jurke and T. Weigand, Global F-theory GUTs, Nucl. Phys. B 829 (2010) 325 [arXiv:0908.1784] [SPIRES].

[29] J. Marsano, N. Saulina and S. Schäfer-Nameki, Monodromies, Fluxes and Compact Three-Generation F-theory GUTs, JHEP 08 (2009) 046 [arXiv: 0906.4672] [SPIRES].

[30] C. Cordova, Decoupling Gravity in F-theory, arXiv:0910.2955 [SPIRES].

[31] J. Marsano, N. Saulina and S. Schäfer-Nameki, Compact F-theory GUTs with U(1)PQ, JHEP 04 (2010) 095 [arXiv: 0912.0272] [SPIRES].

[32] T.W. Grimm, S. Krause and T. Weigand, F-Theory GUT Vacua on Compact Calabi-Yau Fourfolds, JHEP 07 (2010) 037 [arXiv: 0912.3524] [SPIRES].

[33] T.W. Grimm, The $N=1$ effective action of F-theory compactifications, Nucl. Phys. B 845 (2011) 48 [arXiv: 1008.4133] [SPIRES].

[34] E. Dudas and E. Palti, On hypercharge flux and exotics in F-theory GUTs, JHEP 09 (2010) 013 [arXiv: 1007.1297] [SPIRES].

[35] H. Abe, T. Kobayashi and H. Ohki, Magnetized orbifold models, JHEP 09 (2008) 043 [arXiv: 0806.4748] [SPIRES].

[36] H. Abe, K.-S. Choi, T. Kobayashi and H. Ohki, Three generation magnetized orbifold models, Nucl. Phys. B 814 (2009) 265 [arXiv:0812. 3534] [SPIRES].

[37] J.P. Conlon, A. Maharana and F. Quevedo, Wave Functions and Yukawa Couplings in Local String Compactifications, JHEP 09 (2008) 104 [arXiv:0807.0789] [SPIRES].

[38] P. Di Vecchia, A. Liccardo, R. Marotta and F. Pezzella, Kähler Metrics and Yukawa Couplings in Magnetized Brane Models, JHEP 03 (2009) 029 [arXiv:0810.5509] [SPIRES].

[39] P.G. Camara and F. Marchesano, Open string wavefunctions in flux compactifications, JHEP 10 (2009) 017 [arXiv:0906.3033] [SPIRES].

[40] P.G. Camara and F. Marchesano, Physics from open string wavefunctions, PoS (EPS-HEP 2009) 390 [SPIRES].

[41] F. Marchesano, P. McGuirk and G. Shiu, Chiral matter wavefunctions in warped compactifications, JHEP 05 (2011) 090 [arXiv: 1012.2759] [SPIRES].

[42] D. Cremades, L.E. Ibáñez and F. Marchesano, Yukawa couplings in intersecting D-brane models, JHEP 07 (2003) 038 [hep-th/0302105] [SPIRES].

[43] R.C. Myers, Dielectric-branes, JHEP 12 (1999) 022 [hep-th/9910053] [SPIRES].

[44] A. Font and F. Quevedo, N=1 Supersymmetric truncations and the superstring low-energy effective theory, Phys. Lett. B 184 (1987) 45 [SPIRES].

[45] A. Dymarsky and L. Martucci, D-brane non-perturbative effects and geometric deformations, JHEP 04 (2011) 061 [arXiv: 1012.4018] [SPIRES]. 
[46] M. Graña and J. Polchinski, Supersymmetric three-form flux perturbations on $A d S_{5}$, Phys. Rev. D 63 (2001) 026001 [hep-th/0009211] [SPIRES].

[47] B. Heidenreich, L. McAllister and G. Torroba, Dynamic SU(2) Structure from Seven-branes, JHEP 05 (2011) 110 [arXiv: 1011.3510] [SPIRES].

[48] L. Martucci, D-branes on general $N=1$ backgrounds: Superpotentials and D-terms, JHEP 06 (2006) 033 [hep-th/0602129] [SPIRES].

[49] H. Georgi and C. Jarlskog, A New Lepton-Quark Mass Relation in a Unified Theory, Phys. Lett. B 86 (1979) 297 [SPIRES].

[50] J.R. Ellis and M.K. Gaillard, Fermion Masses and Higgs Representations in SU(5), Phys. Lett. B 88 (1979) 315 [SPIRES].

[51] J.P. Conlon, D. Cremades and F. Quevedo, Kähler potentials of chiral matter fields for Calabi-Yau string compactifications, JHEP 01 (2007) 022 [hep-th/0609180] [SPIRES].

[52] L. Aparicio, D.G. Cerdeño and L.E. Ibáñez, Modulus-dominated SUSY-breaking soft terms in F-theory and their test at LHC, JHEP 07 (2008) 099 [arXiv: 0805.2943] [SPIRES].

[53] H. Fritzsch and Z.-z. Xing, Mass and flavor mixing schemes of quarks and leptons, Prog. Part. Nucl. Phys. 45 (2000) 1 [hep-ph/9912358] [SPIRES].

[54] Particle Data Group collaboration, W.M. Yao et al., Review of particle physics, J. Phys. G 33 (2006) 1.

[55] M.R. Douglas and S. Kachru, Flux compactification, Rev. Mod. Phys. 79 (2007) 733 [hep-th/0610102] [SPIRES].

[56] F. Denef, Les Houches Lectures on Constructing String Vacua, arXiv:0803.1194 [SPIRES].

[57] I. Brunner, M.R. Douglas, A.E. Lawrence and C. Romelsberger, D-branes on the quintic, JHEP 08 (2000) 015 [hep-th/9906200] [SPIRES].

[58] M. Bertolini, M. Billó, A. Lerda, J.F. Morales and R. Russo, Brane world effective actions for D-branes with fluxes, Nucl. Phys. B 743 (2006) 1 [hep-th/0512067] [SPIRES].

[59] I. Antoniadis, A. Kumar and B. Panda, Fermion Wavefunctions in Magnetized branes: Theta identities and Yukawa couplings, Nucl. Phys. B 823 (2009) 116 [arXiv:0904.0910] [SPIRES].

[60] P.G. Cámara, C. Condeescu and E. Dudas, Holomorphic variables in magnetized brane models with continuous Wilson lines, JHEP 04 (2010) 029 [arXiv:0912.3369] [SPIRES].

[61] A. Kapustin, Topological strings on noncommutative manifolds, Int. J. Geom. Meth. Mod. Phys. 1 (2004) 49 [hep-th/0310057] [SPIRES].

[62] V. Pestun, Topological strings in generalized complex space, Adv. Theor. Math. Phys. 11 (2007) 399 [hep-th/0603145] [SPIRES].

[63] N. Seiberg and E. Witten, String theory and noncommutative geometry, JHEP 09 (1999) 032 [hep-th/9908142] [SPIRES]. 